\title{
MicroRNA biogenesis and expression in the anoxia-tolerant red-eared slider turtle, Trachemys scripta elegans
}

\author{
By \\ Sarah A. Breedon

\footnotetext{
Master of Science

Department of Biology
} \\ Carleton University \\ Ottawa, Ontario, Canada \\ (C) Copyright 2021 \\ Sarah A. Breedon
}

B.Sc. Honours, University of Western Ontario, 2019

A Thesis Submitted to the Faculty of Graduate Studies and Research in Partial Fulfillment of the Requirements for the Degree of 


\section{ABSTRACT}

The red-eared slide turtle (Trachemys scripta elegans) is able to survive prolonged episodes of anoxia without suffering any apparent damage. This feat is underscored by a complex set of regulatory mechanisms to achieve metabolic rate depression (MRD) including microRNA (miRNA)-mediated gene silencing. Immunoblotting of relative protein abundance was used to analyze miRNA biogenesis under anoxic and reoxygenated conditions in liver and muscle, finding tissue-specific regulation of miRNA production. Cytoplasmic granule proteins were also assessed to inspect mRNA fate, the results of which indicated that mRNA sequestration to stress granules or processing bodies was also tissue specific. Next, the miRNAome was analyzed using RNA sequencing and bioinformatic analyses, revealing anoxia-induced suppression of processes associated with cell cycle progression and protein turnover. Altogether, these results indicate that miRNA targeting of mRNA transcripts occurs in a tissue specific manner with a particular focus on the suppression of energetically expensive processes. 


\section{PREFACE}

This integrated M.Sc. thesis is composed of two main research papers that are currently awaiting submission:

\section{Chapter 2:}

Breedon, S.A. and Storey, K.B. (2021). Lost in translation: exploring the regulation of microRNA biogenesis and messenger RNA fate in the anoxia-tolerant red-eared slider turtle.

\section{Chapter 3:}

Breedon, S.A. and Storey, K.B. (2021). MicroRNA expression profiling in the liver of the red-eared slider turtle, Trachemys scripta elegans. 


\section{ACKNOWLEDGEMENTS}

First and foremost, I would like to thank my supervisor, Ken Storey, for giving me a chance in his lab, even though I had no research experience prior to starting here. I am immensely grateful that you were willing to take me in and give me the guidance necessary to grow into an actual researcher. Your mentorship has provided invaluable experience and knowledge that I will carry forward into my $\mathrm{PhD}$ here and my future career beyond. Thank you for all of your scientific and life advice (even if it's sometimes questionable). Next, I need to thank Jan Storey - thank you for all your patience, hard work, and endless editing. You truly are the backbone of our lab and it wouldn't be what it is without you. I also need to thank my amazing mentors for teaching me all the techniques I used to complete this work, particularly Hanane for showing me how to actually work with miRNA and helping me figure out my thesis project—without your help, I'd probably still be trying to figure out how to get all the code to work. I would also like to thank my friends and family for supporting me through six years of university (only four more to go!), and all of the Storey lab members for making this place such a fun and engaging place to work. Special shoutout to my fellow AAAS Office members, Anchal, Aakriti, and Aline (and honorary members Stuart and Mairelys) for being the best officemates a girl could ask for. From laughing at the idiots on the Wall of Shame, to accidently breaking traffic laws while getting takeout, the fun times in our office helped keep me insane through all the stresses of grad school. I wish you all the best in your current research and whatever you end up doing after you leave the Storey lab, but in the immortal words of Anchal, always remember to "behave yourself!" Lastly, I would like to thank Satan, without whom none of this would have been possible-Ave Satana.

In a race between a tortoise and a hare, I'm a snail.

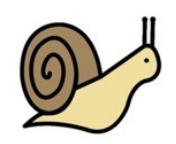




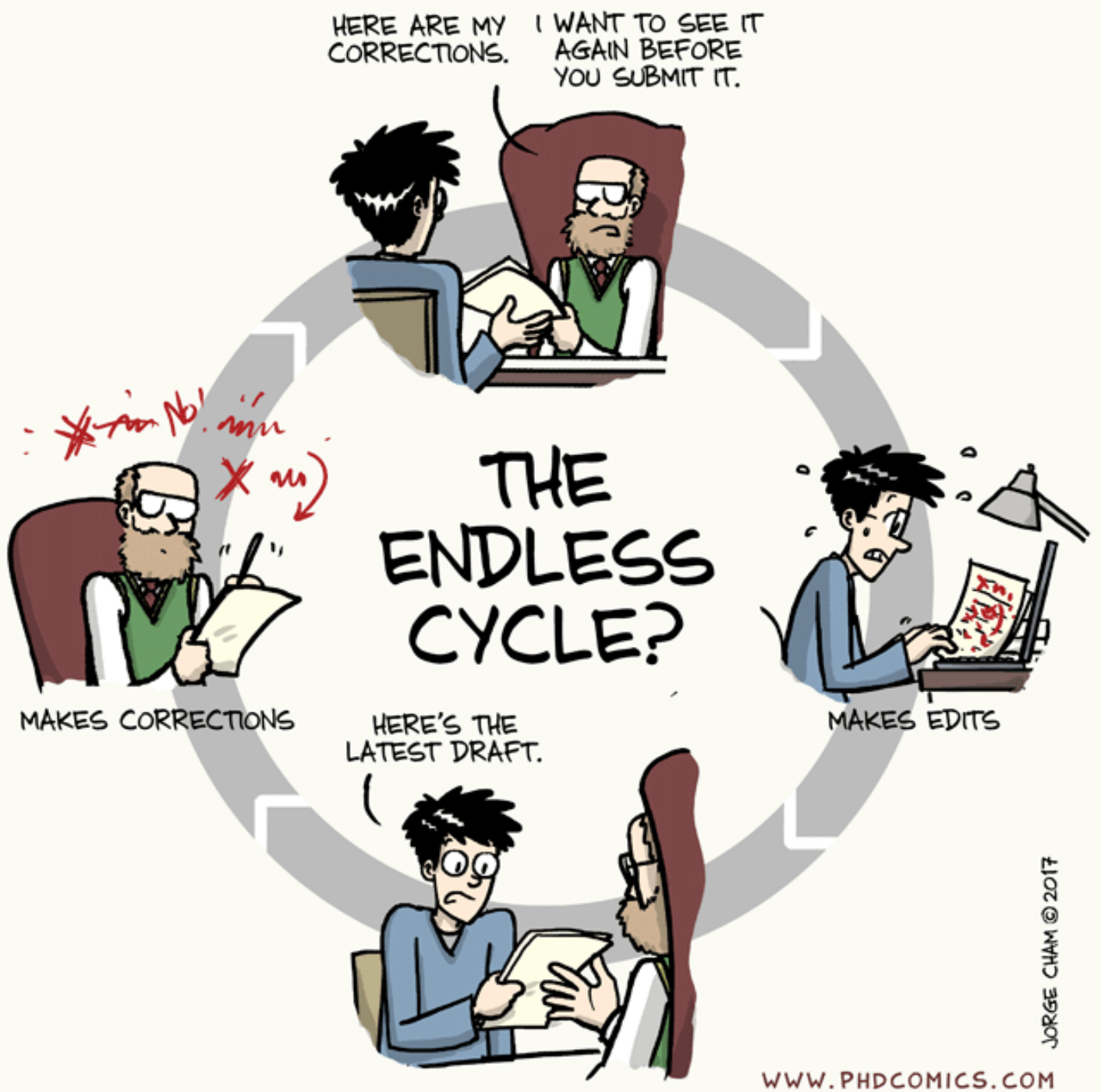




\section{TABLE OF CONTENTS}

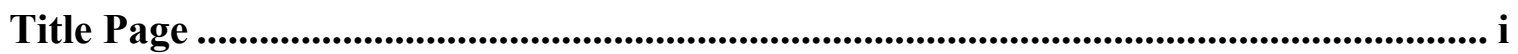

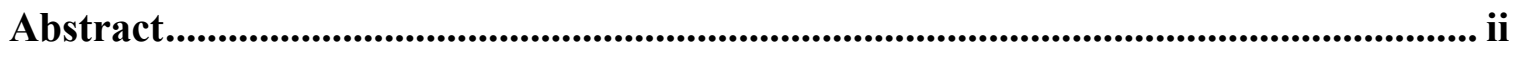

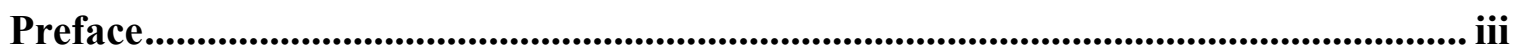

Acknowledgements ............................................................................................................ iv

Table of Contents ..................................................................................................... vi

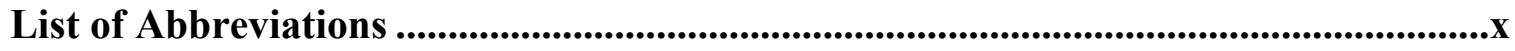

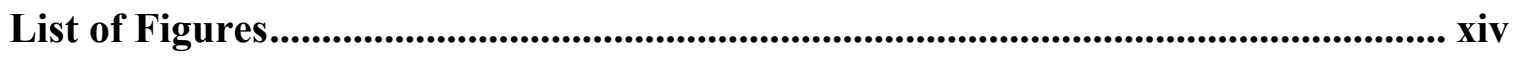

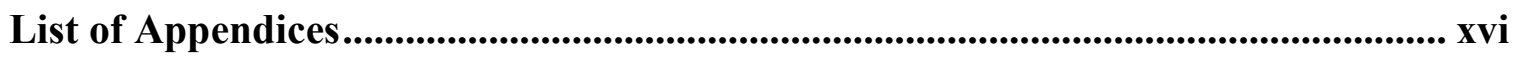

Chapter 1: General Introduction ...................................................................................1

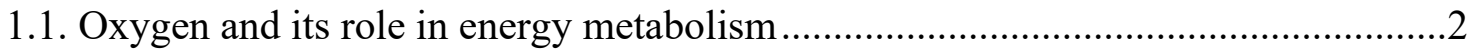

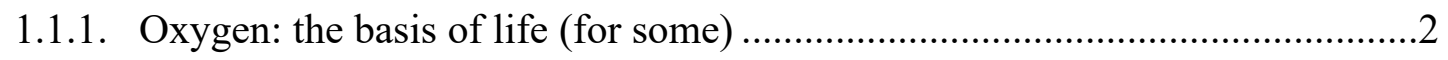

1.1.2. To breathe... (aerobic energy metabolism) .................................................

1.1.3. ...or not to breathe, that is the question (anaerobic energy metabolism) ........6

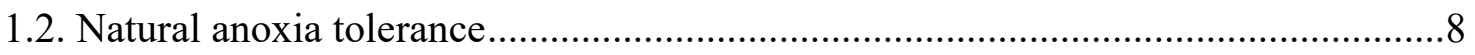

1.3. Adaptations in Trachemys scripta elegans to survive anoxia .............................10

1.3.1. Enhancement of cellular defence mechanisms ........................................ 12

1.3.2. Hypometabolism and metabolic reorganization .......................................16

1.4. Post-transcriptional regulation of gene expression via microRNA .......................23

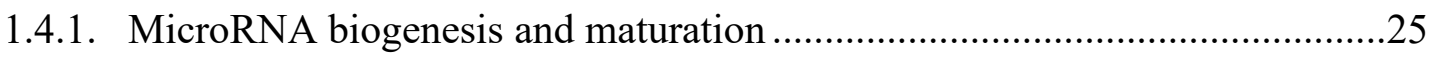

1.4.2. MicroRNA-mediated messenger RNA silencing ......................................28

1.5. Stress granules, P-bodies, and mRNA fate following microRNA binding .............30 
1.5.1. Stress granules as sites of mRNA storage

1.5.2. Processing bodies as sites of mRNA storage and decay..............................32

1.5.3. The interplay between stress granules, processing bodies, and microRNA ...33

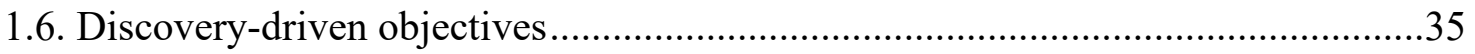

1.6.1. Objective 1: Examine expression of miRNA biogenesis and cytoplasmic granule proteins

1.6.2. Objective 2: Examine miRNA expression and predict corresponding downstream effects

1.7. Figures

Chapter 2: MicroRNA Biogenesis and Messenger RNA Fate ....................................47

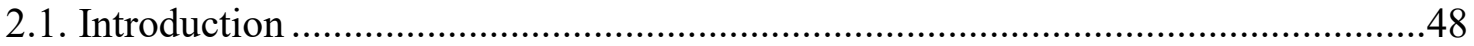

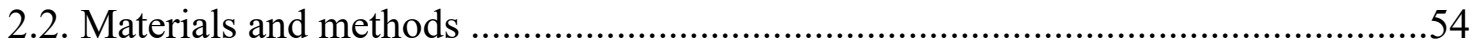

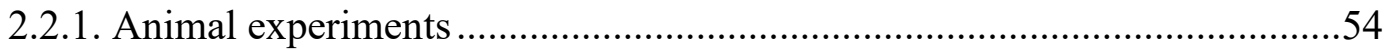

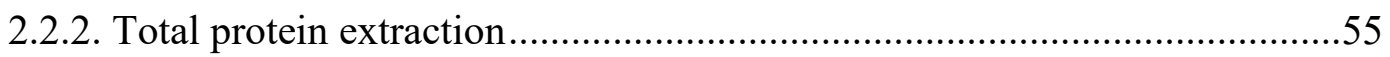

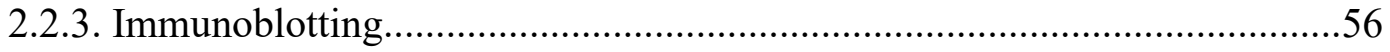

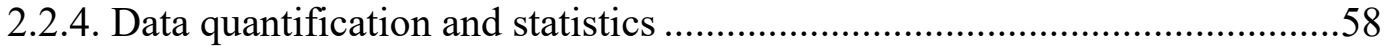

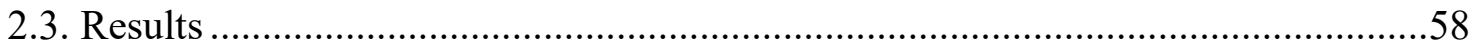

2.3.1. Liver microRNA biogenesis protein expression ....................................58

2.3.2. Muscle microRNA biogenesis protein expression...................................59

2.3.3. Liver P-body and stress granule protein expression ...............................60

2.3.3. Muscle P-body and stress granule protein expression ............................60

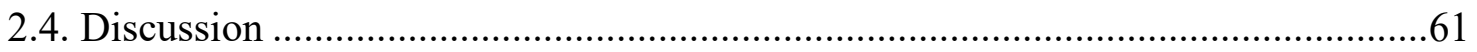

2.4.1. Regulation of microRNA biogenesis .................................................61

2.4.2. Messenger RNA fate in P-bodies and stress granules .............................68

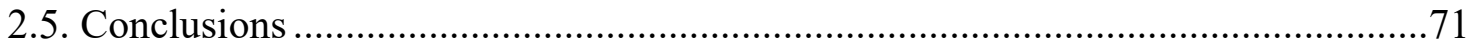


2.6. Figures

Chapter 3: MicroRNA Expression in Anoxic Turtle Liver ...........................................77

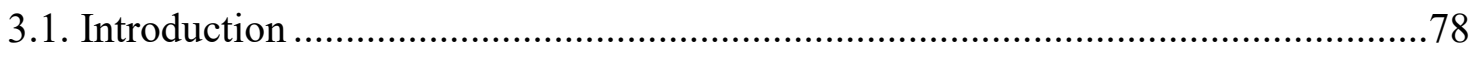

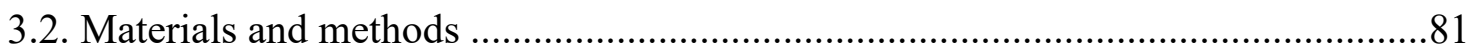

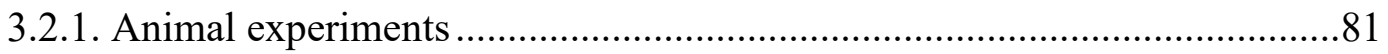

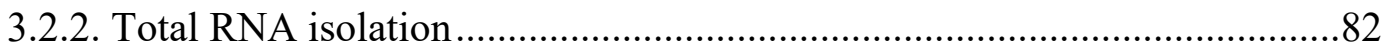

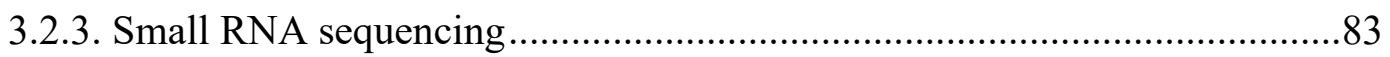

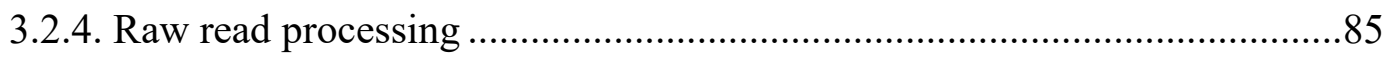

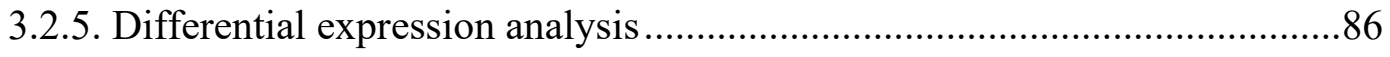

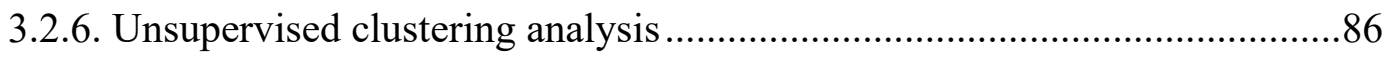

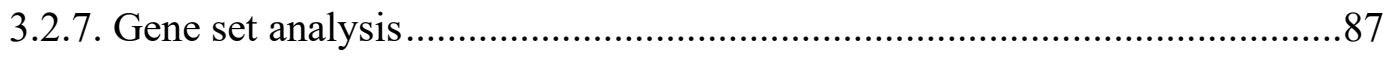

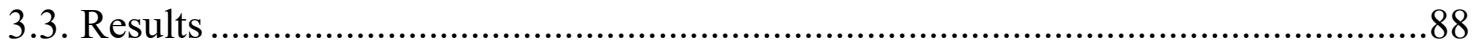

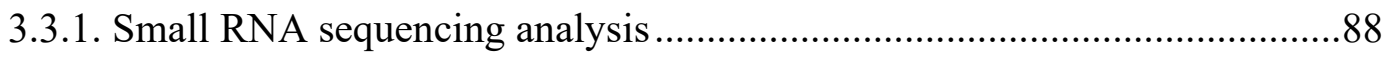

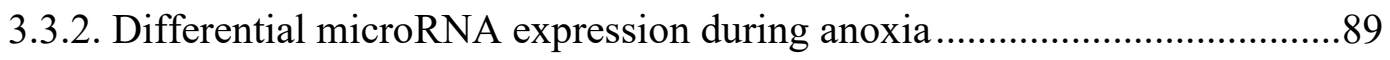

3.3.3. Hierarchical clustering and principal component analysis ........................89

3.3.4. Gene set analysis for differentially expressed microRNA........................90

3.4. Discussion ............................................................................................ 91

3.4.1. MicroRNA expression trends during anoxia .....................................91

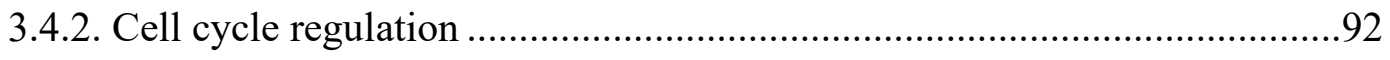

3.4.3. Protein modification and turnover .......................................................93

3.4.4. Olfactory receptors and stimulus detection.......................................96

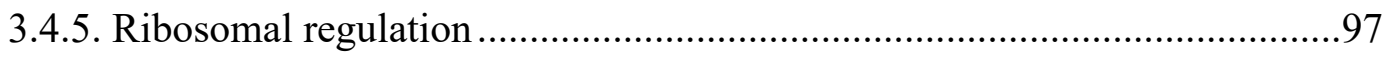

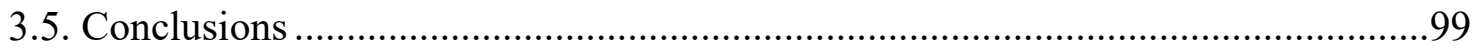

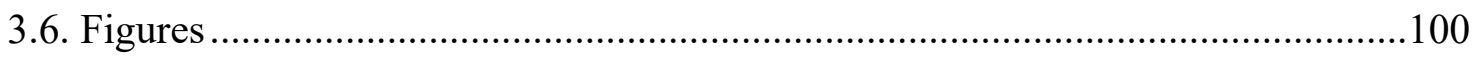


4.1. Red-eared slider turtles and anoxia .....

4.2. Role of microRNAs in the stress response to anoxia

4.3. Future directions

4.3.1. Controlling microRNA transcription

4.3.2. Messenger RNA fate following microRNA targeting

4.3.3. Effect of microRNA regulation on downstream pathways

4.3.4. Beyond microRNA regulation

4.4. Significance and potential applications.

References 


\section{LIST OF ABBREVIATIONS}

$\cdot \mathrm{OH}$

ADP

AGO

ANOVA

APS

ATP

BLAST

bp

CAC

cDNA

CDS

CoA

$\operatorname{ddH}_{2} \mathrm{O}$

DGCR8

DHAP

DNA

DNMT

dNTP

dsRNA

eBayes

ECL

EDTA

eIF

ETC

$\mathrm{FADH}_{2}$
Hydroxyl radicals

Adenosine diphosphate

Argonaute

Analysis of variance

Ammonium persulfate

Adenosine triphosphate

Basic local alignment search tool

Base pair

Citric acid cycle

Complementary DNA

Coding sequence

Coenzyme A

Double-distilled water

DiGeorge syndrome critical region gene 8

Dihydroxyacetone phosphate

Deoxyribonucleic acid

DNA methyltransferase

Deoxynucleotide

Double-stranded RNA

Empirical Bayesian

Enhanced chemiluminescence

Ethylenediamine tetra acetic acid

Eukaryotic initiation factor

Electron transport chain

Reduced flavin adenine dinucleotide 


$\begin{array}{ll}\text { FC } & \text { Fold-change } \\ \text { FDR } & \text { False discovery rate } \\ \text { G3BP1 } & \text { Ras GTPase Activating Protein Binding Protein 1 } \\ \text { GO } & \text { Gene ontology } \\ \text { GST } & \text { Glutathione S-transferase } \\ \text { H }^{+} & \text {Hydrogen ion } \\ \text { H2O }_{2} & \text { Hydrogen peroxide } \\ \text { HEPES } & \text { N-(2-hydroxyethyl) piperazine-N'-(2-ethanesulfonic acid) } \\ \text { HIF-1 } \boldsymbol{\alpha} & \text { Hypoxia inducible factor 1 subunit alpha } \\ \text { HO-1 } & \text { Heme oxygenase-1 } \\ \text { HRP } & \text { Horseradish peroxidase } \\ \text { HSP } & \text { Heat shock protein } \\ \text { IRI } & \text { Ischemia-reperfusion injury } \\ \text { kb } & \text { kilobase } \\ \text { kDa } & \text { Kilo Dalton } \\ \text { KEGG } & \text { Kyoto Encyclopedia of Genes and Genomes } \\ \text { limma } & \text { Linear models for microarray data } \\ \text { miRNA } & \text { MicroRNA } \\ \text { miRNA* } & \text { MicroRNA passenger strand } \\ \text { MRD } & \text { Metabolic rate depression } \\ \text { mRNA } & \text { Messenger RNA } \\ \text { mRNP } & \text { Messenger ribonucleoprotein } \\ \text { NAD } & \text { Nicotinamide adenine dinucleotide } \\ \text { NADH } & \text { Reduced nicotinamide adenine dinucleotide } \\ \text { NCBI } & \text { National Center for Biotechnology Information } \\ \text { NGS } & \text { Next generation sequencing } \\ \text { nMomolar }\end{array}$




\begin{tabular}{ll} 
Nrf2 & Nuclear factor-erythroid 2 related factor -2 \\
nt & Nucleotide \\
O $^{-}$ & Superoxide anions \\
O $^{-2}$ & Peroxide \\
ODDD & Oxygen-dependent degradation domain \\
OH$^{-}$ & Hydroxyl ions \\
OR & Olfactory receptor \\
P-body & Processing body \\
PABPC1 & Poly(A) Binding Protein Cytoplasmic 1 \\
PACT & Protein kinase RNA activator \\
PAGE & Polyacrylamide gel electrophoresis \\
PCR & Polymerase chain reaction \\
PEP & Phosphoenolpyruvate \\
PMSF & Phenylmethylsulfonyl fluoride \\
pO2 & Partial pressure of oxygen \\
Pre-miRNA & Precursor microRNA \\
Pri-miRNA & Primary microRNA \\
PTM & Post-translational modification \\
PVDF & Polyvinylidene fluoride \\
RBP & RNA-binding protein \\
RISC & RNA-induced silencing complex \\
RLC & RISC loading complex \\
RNA & Ribonucleic acid \\
RNAi & RNA interference \\
RNase & Ribonuclease \\
RNP & Ribonucleoprotein \\
ROS & Reactive oxygen species \\
\hline
\end{tabular}




\begin{tabular}{ll} 
rRNA & Ribosomal RNA \\
RT & Room temperature $\left(\sim 2{ }^{\circ} \mathrm{C}\right)$ \\
RT-qPCR & Quantitative real time polymerase chain reaction \\
SDS & Sodium dodecyl sulfate \\
ShRNA & Short hairpin RNA \\
siRNA & Small interfering RNA \\
SOD & Superoxide dismutase \\
TBST & Tris-buffered saline and Tween-20 \\
TCA & Tricarboxylic acid \\
TEMED & Tetramethylethylenediamine \\
TIA-1 & T-cell-restricted intracellular antigen-1 \\
TIAR & T-cell-restricted intracellular antigen -related protein \\
TRBP & Transactivation response RNA binding protein \\
Tris & Tris (hydroxymethyl) aminomethane \\
UTR & Untranslated region \\
XPO5 & Exportin 5 \\
XRN1 & $5 '-3 '$ Exoribonuclease 1 \\
\hline
\end{tabular}




\section{LIST OF FIGURES}

Figure 1.1. General overview of eukaryotic aerobic and anaerobic respiration. 39

Figure 1.2. Global range of the red-eared slider turtle (Trachemys scripta elegans) as of 2021.

Figure 1.3. Canonical microRNA biogenesis and maturation pathway. 41

Figure 1.4. MicroRNA targeting of messenger RNA and its translational effects.

Figure 1.5. Stress granule and processing body sequestration of mRNA in 44 response to stress.

Figure 1.6. Experimental procedure for the relative expression analysis of microRNA biogenesis and P-body/stress granule-associated proteins (Chapter 2).

Figure 1.7. Experimental procedure for microRNA sequencing and bioinformatic analyses (Chapter 3).

Figure 2.1. Analysis of miRNA biogenesis pathway protein levels in redeared slider turtle liver under control, anoxia, and recovery conditions.

Figure 2.2. Analysis of miRNA biogenesis pathway protein levels in red muscle of red-eared slider turtles under control, anoxia, and recovery conditions.

Figure 2.3. Relative expression analysis of proteins associated with P-body and stress granule in red-eared slider turtle liver.

Figure 2.4. Relative expression analysis of proteins associated with P-body 76 and stress granule in red-eared slider turtle red muscle. 
Figure 3.1. MicroRNA differential expression (DE) analysis in the liver of 100 Trachemys scripta elegans in response to $20 \mathrm{~h}$ anoxic exposure.

Figure 3.2. Unsupervised clustering analyses in of miRNA species in liver of aerobic control vs $20 \mathrm{~h}$ anoxia exposed Trachemys scripta elegans

Figure 3.3. GO molecular function (MF) term gene set enrichment based on miRNAs identified in DE analysis of liver from anoxic Trachemys scripta elegans relative to controls.

Figure 3.4. GO biological process (BP) term gene set enrichment based on miRNAs identified in DE analysis of liver from anoxic Trachemys scripta elegans relative to controls.

Figure 3.5. GO cellular component $(\mathrm{CC})$ term gene set enrichment based on miRNAs identified in DE analysis of liver from anoxic Trachemys scripta elegans relative to controls.

Figure 3.6. KEGG pathway term gene set enrichment based on miRNAs identified in DE analysis in liver from anoxic Trachemys scripta elegans relative to controls. 


\section{LIST OF APPENDICES}

$\begin{array}{ll}\text { APPENDIX A: } & 153\end{array}$

APPENDIX B: $\quad$ Antibody Supplier Information 155

APPENDIX C: $\quad$ Verifying Antibody Specificity for Immunoblotting 157

APPENDIX D: $\quad$ Optimized Immunoblotting Conditions 159

APPENDIX E: $\quad$ Representative Immunoblot Images 161 


\section{CHAPTER 1 \\ General Introduction}




\subsection{Oxygen and its role in energy metabolism}

\subsubsection{Oxygen: the basis of life (for some)}

Oxygen is the second most abundant gas on Earth, accounting for approximately $21 \%$ of the atmosphere. For much of Earth's history, there was no oxygen in the atmosphere, with levels first rising around 2.4 billion years ago during the Great Oxidation Event. Early cyanobacteria are likely to thank for this critical event; with their ability to convert water, carbon dioxide, and the sun's energy into sugars and oxygen through the process of photosynthesis (Schirrmeister et al., 2015). Although the oxygen produced from photosynthesis is considered a waste product for these organisms, their releasing of oxygen converted Earth's atmosphere into an environment capable of sustaining complex multicellular aerobic life. Aerobic organisms evolved to utilize oxygen as the final acceptor of electrons in their electron transport chain (ETC), enabling increased energy output compared to anaerobic catabolism (Embley \& Martin, 2006). This is due to the high redox potential of oxygen, which allows it to easily accept electrons from reduced metabolic intermediates, making it a critical substrate in aerobic energy metabolism.

In lung-breathing vertebrates, oxygen is breathed into the lungs where it moves from the alveoli into the capillaries which surround each alveolus via diffusion (Longeville \& Stingaciu, 2017). After reaching the capillaries, oxygen diffuses from the plasma into red blood cells (RBCs) and binds to hemoglobin (Longeville \& Stingaciu, 2017). The oxygen-rich RBCs are pumped throughout the body until they reach peripheral tissues, where oxygen diffuses out of the RBCs and into metabolically active cells via a decrease in oxygen affinity (Pittman, 2016). The affinity of hemoglobin for oxygen depends on the local partial pressure of oxygen $\left(\mathrm{pO}_{2}\right)$, with oxygen affinity being high when $\mathrm{pO}_{2}$ is high, 
and low when $\mathrm{pO}_{2}$ is low (Pittman, 2016; Popel, 1989). In the alveolar-capillary junction, $\mathrm{pO}_{2}$ is high, allowing oxygen to be quickly loaded into hemoglobin, while in peripheral tissues, $\mathrm{pO}_{2}$ is low, aiding in the release of oxygen via the decreased affinity of hemoglobin (Longeville \& Stingaciu, 2017; Patel \& Mohiuddin, 2020). Once released, oxygen diffuses into the cells and eventually into the mitochondria where it can be used for aerobic respiration (Pittman, 2016).

While oxygen-linked catabolism results in increased net energy production, the reliance on oxygen means that the organism is sensitive to oxygen deprivation, including hypoxic (low oxygen) or anoxic (no oxygen) conditions. During periods of little to no oxygen, an aerobic organisms' metabolic demand for oxygen exceeds the available supply, which rapidly leads to severe cellular/tissue damage and, eventually, death of the organism (Brierley, 1977). This underlies the sensitivity to oxygen deprivation of most vertebrateshumans included — which are particularly prone to organ damage following even short bouts of oxygen deprivation. However, some extraordinary aerobic organisms have adapted to oxygen-deprived environments allowing them to survive periods of limited oxygen from a few hours to a few months without obtaining any deleterious physiological effects (Larson et al., 2014; Storey, 2007; Storey \& Storey, 1990). An overview of energy metabolism under aerobic and anaerobic conditions can be seen in Figure 1.1.

\subsubsection{To breathe... (aerobic energy metabolism)}

Under normoxic (normal oxygen) conditions, aerobic eukaryotic respiration begins with glycolysis in the cytoplasm. Glycolysis is a series of reactions that splits the six-carbon glucose into two three-carbon pyruvate molecules. Briefly, this process begins with the energy-requiring phase whereby the initial glucose molecule is phosphorylated into 
glucose-6-phosphate, which is then isomerized into fructose-6-phosphate (Melkonian \& Schury, 2019). Fructose-6-phosphate is then phosphorylated into fructose-1,6bisphosphate, which cleaves to form dihydroxyacetone phosphate (DHAP) and glyceraldehyde-3-phosphate, and DHAP is subsequently isomerized into a second glyceraldehyde-3-phosphate molecule (Melkonian \& Schury, 2019). Next, the energyreleasing phase begins with each glyceraldehyde-3-phosphate molecule being oxidized into 1,3-bisphosphoglycerate through the reduction of $\mathrm{NAD}^{+}$into $\mathrm{NADH}$ and $\mathrm{H}^{+}$ (Melkonian \& Schury, 2019). A phosphate group from 1,3-bisphosphoglycerate is then donated to adenosine diphosphate (ADP) to form adenosine triphosphate (ATP) and 3phosphoglycerate (Melkonian \& Schury, 2019). Next, 3-phosphoglycerate is isomerized in 2-phosphoglycerate, which is dehydrated to form phosphoenolpyruvate (PEP) (Melkonian \& Schury, 2019). PEP is highly unstable, and as such, it readily donates its phosphate group to ADP to form ATP, converting PEP into pyruvate (Melkonian \& Schury, 2019). Overall, glycolysis requires one glucose molecule and two ATP molecules to produce four ATP molecules (a net gain of two ATP), two pyruvate molecules, and four NADH molecules (Melkonian \& Schury, 2019). Though glycolysis is critical for aerobic energy metabolism, it is an inherently anaerobic process, and therefore, anaerobic organisms also use glycolysis during ATP synthesis.

Following glycolysis, the newly formed NADH and pyruvate are sent to the mitochondria (the powerhouse of the cell) where the vast majority of ATP synthesis occurs. NADH is shuttled into the ETC to create ATP and be recycled back into glycolysis (explained further below). Pyruvate, on the other hand, must first join the tricarboxylic acid (TCA) cycle (also known as the citric acid cycle [CAC] or the Krebs cycle) (Haddad \& 
Mohiuddin, 2019). The TCA cycle starts with the oxidation of pyruvate into acetylCoenzyme A (CoA), which produces one NADH molecule and releases carbon dioxide (Haddad \& Mohiuddin, 2019). From here, acetyl-CoA is joined by oxaloacetate, forming a citrate molecule and causing the CoA group to release (Haddad \& Mohiuddin, 2019). Citrate is then isomerized into isocitrate, which is oxidized to $\alpha$-ketoglutarate through the reduction of NAD ${ }^{+}$to NADH (Haddad \& Mohiuddin, 2019). Similarly, $\alpha$-ketoglutarate is oxidized, reducing $\mathrm{NAD}^{+}$to $\mathrm{NADH}$, and then picks up $\mathrm{CoA}$ to form succinyl $\mathrm{CoA}$; however, this molecule is unstable, so it rapidly replaces the CoA group with a phosphate group (Haddad \& Mohiuddin, 2019). This phosphate group is subsequently transferred to ADP to form an ATP molecule—-some cells utilize guanosine diphosphate (GDP) instead of ADP, forming guanosine triphosphate (GTP) instead of ATP—leaving behind a succinate molecule (Haddad \& Mohiuddin, 2019). The oxidation of succinate forms fumarate through the transfer of $\mathrm{H}^{+}$atoms to FAD, creating $\mathrm{FADH}_{2}$ (Haddad \& Mohiuddin, 2019). Next, water is added to fumarate to convert it into malate, which can then be oxidized to reform oxaloacetate to restart the cycle (Haddad \& Mohiuddin, 2019). Overall, the TCA cycle produces two ATP molecules, six molecules of NADH, and one $\mathrm{FADH}_{2}$ molecule from one molecule of glucose.

All of the electron carriers ( $\mathrm{NADH}$ and $\left.\mathrm{FADH}_{2}\right)$ produced from glycolysis and the TCA cycle are utilized in the ETC to make additional ATP through oxidative phosphorylation. This process begins with the oxidation of $\mathrm{NADH}$ and $\mathrm{FADH}_{2}$, causing them to release high energy electrons that are passed through the transmembrane proteins of the ETC, and $\mathrm{H}^{+}$protons, which are pumped from the mitochondrial matrix into the intermembrane space using the energy lost during the aforementioned electron transfer 
(Deshpande \& Mohiuddin, 2020). The buildup of $\mathrm{H}^{+}$ions in the intermembrane space creates the electrochemical gradient (proton-motive force), which is responsible for the diffusion of $\mathrm{H}^{+}$back into the matrix (chemiosmosis) through the transmembrane enzyme ATP synthase (Deshpande \& Mohiuddin, 2020). This proton diffusion causes ATP synthase to rotate, catalyzing the addition of a phosphate group to ADP, forming ATP (Deshpande \& Mohiuddin, 2020). Following ATP production, electrons that have been passed through the ETC are transferred to oxygen molecules in the matrix, and then free $\mathrm{H}^{+}$ions join to form water (Deshpande \& Mohiuddin, 2020). Overall, oxidative phosphorylation is responsible for the regeneration of the electron carriers necessary for glycolysis and the TCA cycle, which altogether generate approximately 30-32 total ATP from one glucose molecule during aerobic respiration (exact numbers vary depending on the differential use of metabolic intermediates) (Deshpande \& Mohiuddin, 2020; Melkonian \& Schury, 2019). Given how advantageous aerobic metabolism is in terms of total energy output, it is no wonder that most eukaryotic life on Earth has evolved to utilize oxygen as a critical component of ATP synthesis. However, even aerobic organisms sometimes experience periods of limited oxygen availability, for which they have developed a complex set of processes that maintain basic cellular functionality until oxygen availability increases again.

\subsection{3. ...or not to breathe, that is the question (anaerobic energy metabolism)}

During hypoxic or anoxic conditions, a cell's potential for ATP synthesis drastically decreases. For facultative anaerobes, glycolysis continues as in normoxic conditions because it is not dependent on the presence of oxygen, and, as such, a small amount of ATP synthesis still occurs (Melkonian \& Schury, 2019). However, the main 
difference arises during oxidative phosphorylation, which depends on oxygen as the final electron acceptor of the ETC. Whenever the supply of oxygen is disrupted, the ETC halts because it becomes backlogged with de-energized electrons, thus preventing electron carriers from depositing new, higher energy electrons into the ETC (Bagali et al., 2018). This also blocks the production of $\mathrm{H}^{+}$ions, which maintains the electrochemical gradient necessary for chemiosmosis occur (Bagali et al., 2018). Additionally, the halting of oxidative phosphorylation prevents the regeneration of $\mathrm{NAD}^{+}$, which is required for normal functioning of glycolysis and the TCA cycle. In order to ensure that glycolysis continues, the conversion of $\mathrm{NADH}$ to $\mathrm{NAD}^{+}$needs to occur using anaerobic fermentation.

There are two main types of anaerobic fermentation: ethanol fermentation, or lactic acid fermentation. Though these pathways generate different metabolites from pyruvate, they both serve the critical function of regenerating the electron carriers needed for ATP synthesis. In ethanol fermentation, pyruvate is converted into acetaldehyde via the removal of a carboxyl group, and the acetaldehyde is then able to accept an electron from NADH to regenerate $\mathrm{NAD}^{+}$, forming ethanol as a by-product (Storey \& Storey, 1990). During lactic acid fermentation, $\mathrm{NADH}$ transfers its electrons to pyruvate to regenerate $\mathrm{NAD}^{+}$, forming lactate as a by-product (Storey \& Storey, 1990; Warren \& Jackson, 2008).

These fermentation processes are critical for anaerobic energy production; however, fermentation converts pyruvate into alternative metabolites instead of acetylCoA, both of which are unable to be used in the TCA cycle, leaving glycolysis as the only ATP source during anaerobic cellular respiration. This is problematic because glycolysis does not produce nearly enough ATP-two per glucose molecule compared to the 30-32 ATP total from aerobic respiration - to sustain all of the biochemical reactions necessary 
for a complex multicellular organism to function normally, thus leading to cell death and eventually organismal death if the anoxic episode is prolonged. Even short bouts of oxygen deprivation can disrupt normal function of energetically expensive processes such as protein turnover (production and degradation), gluconeogenesis, and ion motive ATPase pumping (Hochachka \& Lutz, 2001). Hence, facultative anaerobes typically only use anaerobic respiration as a last resort for energy production during periods of oxygen deprivation and switch back to aerobic respiration whenever oxygen is reintroduced. However, some animals have developed complex adaptations that enable them to endure prolonged periods of little to no oxygen. These incredible organisms have numerous physiological and biochemical mechanisms through which they are able to mitigate the buildup of harmful metabolites, reduce their need for cellular energy, and reprioritize pathways necessary for anoxia survival (Storey, 2007).

\subsection{Natural anoxia tolerance}

Across phyla, many animals are faced with various environmental stress that challenge their day-to-day life, such as extreme high or low temperatures, water and food scarcity, and limited oxygen availability (Storey \& Storey, 1990, 2004). Oxygen limitation is a common stress that is naturally experienced by a wide variety of animals, including mammals, amphibians, invertebrates, fish, birds, and reptiles (Storey, 2007; Storey \& Storey, 1990; Vornanen et al., 2009). Variations in oxygen availability may occur due to changes in environmental oxygen that limit an animal's air supply (e.g. increasing altitude, oxygen depleted sediments, hypoxic/anoxic bodies of water, ice-locked ponds), or because of behaviours that temporarily interrupts their access to oxygen (e.g. aerial exposure of gill-breathers, breath-hold diving of lung-breathers) (Storey, 1996). The duration of 
oxygen stresses can be brief, usually experienced on a daily basis due to a behavioural trait of the animal, or long-term, typically extending over an entire season due to environmental pressures. These limitations can be classified as either decreased oxygen levels (hypoxia), or the total lack of oxygen (anoxia). The ability to withstand prolonged hypoxia or anoxia exposure is remarkable considering oxygen depletion is rapidly lethal for most animals. Hence, the ability to mitigate this damage is a vital adaptation to animals that are commonly exposed to hypoxic or anoxic episodes.

In order to survive bouts of hypoxia or anoxia, animals employ numerous physiological and biochemical adaptations. Some relatively common physiological responses to short-term hypoxic conditions in vertebrates include increased lung ventilation, increasing heart rate to pump oxygenated blood throughout the body, alterations in hemoglobin-oxygen affinity, and releasing the spleen's stored RBCs (Jackson, 2002; Storey, 2007; Storey \& Storey, 2004). These physiological adaptations result in increased oxygen uptake and improved delivery of oxygen-rich blood to critical organs (Storey, 2007; Storey \& Storey, 2004). However, these adaptations are only suitable for short-term use, and are not sustainable in the event of long-term hypoxic/anoxic episodes (Storey, 2007; Storey \& Storey, 2004). Additional biochemical responses are also activated in response to oxygen deprivation including increased liver glycogen breakdown through glycolysis and the utilization of creatine phosphate reserves to generate ATP (Gaddi et al., 2017; Storey \& Storey, 2004). These physiological and biochemical adaptations are often enough to ensure organismal survival of short-term and/or mild hypoxic episodes; however, extended bouts of total anoxia require additional biochemical adaptations, as well as a more intensive reorganization of metabolic energetics. 


\subsection{Adaptations in Trachemys scripta elegans to survive anoxia}

The red-eared slider turtle (Trachemys scripta elegans) is one of the best examples of a vertebrate that is able to survive extended periods of oxygen deprivation without suffering any apparent long-term damage. While the red-eared slider turtle is native to the southern United States and northern Mexico, it has been introduced into non-native habitats the world over (Figure 1.2) (Global Biodiversity Information Facility, 2021). In fact, due to their ability to adapt to a wide variety of habitats and diets, these turtles have spread to climates completely different to their natural habitat, including northern ranges where the temperatures regularly drop below freezing. However, their penchant for adaptability makes them of considerable interest in the study of natural adaptations to survive extreme environmental stressors, including limited oxygen availability.

During their normal day-to-day activities, turtles engage in breath-hold diving in order to find food or escape predation, surfacing periodically for fresh air in between dives (Clark \& Miller, 1973; Jackson, 1968; Storey, 2007). This exposes them to sporadic bursts of hypoxic conditions until they are able to surface from these short-term dives. The frequency that these hypoxic conditions are encountered hints at the robust adaptations the red-eared slider turtle must have so that they can endure this repeated oxygen deprivation. However, these turtles also experience lack of oxygen on a much larger scale. In particular, red-eared slider turtles in northern climates will brumate underwater in ponds and lakes over the winter months in order to escape the extreme freezing terrestrial temperatures of these regions. While this saves them from the worst of the cold, once these bodies of water freeze-over, they have no way of surfacing to receive fresh oxygen until the ice melts in the spring. This leaves approximately 3-5 months where they are exposed to hypoxic or 
complete anoxic conditions at around $3{ }^{\circ} \mathrm{C}$, during which their blood oxygen levels can drop to near zero values (Herbert \& Jackson, 1985; Jackson, 1968; Ultsch, 1985, 1989; Ultsch \& Jackson, 1982). Both behavioural- and environmental-related oxygen deprivation events cause cellular oxygen levels to decrease, eventually leading to the disruption of mitochondrial ATP production and, subsequently, the cellular processes that depend on that ATP generation (Storey, 2007).

To cope with this, facultative anaerobes such as the red-eared slider turtle are able to utilize anaerobic glycolysis to produce enough ATP to fuel the processes necessary for anoxia survival (Storey, 2004). This is supported through extensive organ glycogen stores, chiefly the liver (Jackson \& Ultsch, 2010). However, anaerobiosis requires turtles to have sufficient protective mechanisms as lactate fermentation leads to the accumulation of harmful metabolites that can cause lethal acidosis and anoxia-reoxygenation cycles lead to cellular stress (Storey, 2004). Additionally, red-eared slider turtles have extensive physiological and biochemical adaptive mechanisms that increase their ability to utilize the limited oxygen they have access to, and to survive when that oxygen is eventually depleted (Storey, 2004). Adaptations for oxygen deprivation in red-eared slider turtles and other anoxia-tolerant animals are either reactive-responding immediately to acute, short-term limitations—or preparatory—deploying complex regulatory mechanisms and processes to survive extended anoxic bouts (Giraud-Billoud et al., 2019; Hadj-Moussa \& Storey, 2020; Larson et al., 2014). In either case, these adaptations most critically involve: (1) the enhancement of defence mechanisms and pro-survival pathways to protect against cellular damage and death, and (2) the entrance into a hypometabolic state and metabolic reorganization (Krivoruchko \& Storey, 2010b). 


\subsubsection{Enhancement of cellular defence mechanisms and pro-survival pathways}

During anoxia, the red-eared slider turtle utilizes large liver glycogen stores to fuel the anaerobic production of ATP via lactate fermentation (Storey \& Storey, 1990). This allows the turtle to maintain minimal levels of ATP synthesis in the absence of oxygen; however, this process also produces large amounts of anaerobic metabolites, particularly lactate. As previously explained in Section 1.1.3., in oxygen-deprived cells, pyruvate produced from glycolysis cannot be utilized in the ETC in the mitochondria. The reduction of pyruvate into lactate uses the oxidation of NADH into $\mathrm{NAD}^{+}$, which is necessary for glycolysis to continue as normal (Storey \& Storey, 1990). Though this fermentative conversion of pyruvate to lactate is necessary to maintain critical cellular ATP production, the build-up of metabolites like lactate is ultimately harmful and can lead to lethal acidosis if not adequately managed (Jackson, 2000; Storey, 2007; Storey \& Storey, 1990).

During extended anoxic bouts, plasma lactate concentrations can climb as high as $150-200 \mathrm{mM}$ in some turtle species, leading to a significant decrease in plasma $\mathrm{pH}$ (Davis \& Jackson, 2007; Jackson, 2000; Ultsch \& Jackson, 1982). However, some anoxia-tolerant turtles such as the red-eared slider have extensive mechanisms to cope with excess lactate. The extracellular buffering of lactate with bicarbonate $\left(\mathrm{HCO}_{3}^{-}\right)$is the first line of defence against acidosis (Jackson, 2000; Warren \& Jackson, 2007). However, as the anoxic episode continues, this buffering capacity is exceeded by the sheer amount of lactate produced during anaerobic fermentation (Jackson, 2000; Jackson, Crocker, et al., 2000; Jackson, Ramsey, et al., 2000). The calcium carbonate-rich shell and skeleton of the red-eared slider turtle offer protection from these elevated lactate concentrations and the threat of lethal acidosis via enhanced acid-base buffering (Davis \& Jackson, 2007; Jackson, 2000; 
Jackson, Crocker, et al., 2000). The ossified shell and bones release carbonate minerals into the extracellular fluid where they form complexes with protons and lactate anions to supplement buffering (Davis \& Jackson, 2007; Jackson, 2000; Jackson, Crocker, et al., 2000). Additionally, protons and lactate are absorbed into the shell and bones, where protons are buffered by carbonate and lactate is stored until the restoration of normoxic conditions (Davis \& Jackson, 2007; Jackson, 2000; Jackson, Crocker, et al., 2000). Together, these mechanisms account for over $70 \%$ of the red-eared slider turtle's total lactic acid buffering during extended periods of anoxia and enable it to maintain general plasma homeostasis to avoid lethal acidosis (Jackson, 2000).

Another critical problem the red-eared slider turtle must contend with is the overproduction of reactive oxygen species (ROS). ROS form most commonly during periods of exposure to excessive oxygen concentrations (hyperoxia) and/or the sudden reperfusion of oxygen to cells following a period of hypoxia/anoxia. ROS are natural byproducts of normal cell activity formed during mitochondrial electron transport from the reduction of oxygen. The term ROS covers a wide array of reactive molecules and free radicals derived from molecular oxygen, including hydrogen peroxide $\left(\mathrm{H}_{2} \mathrm{O}_{2}\right)$, superoxide anions $\left(\mathrm{O}_{2}^{-}\right)$, hydroxyl ions $\left(\mathrm{OH}^{-}\right)$and radicals $\left({ }^{\circ} \mathrm{OH}\right)$, and peroxide $\left(\mathrm{O}_{2}{ }^{-2}\right)$ (Blokhina et al., 2003; Storey, 1996). ROS are highly reactive and can damage critical cellular macromolecules such as nucleic acids, proteins, and lipids, which can ultimately lead to metabolic injury and cell death if not properly dealt with (Auten \& Davis, 2009). All aerobic organisms have evolved some level of detoxification mechanisms in order to mitigate the damage caused by ROS (Storey, 1996). Additionally, animals that routinely undergo episodes of oxygen deprivation followed by the sudden reintroduction of oxygen 
must have increased antioxidant defences in order to minimize oxidative injuries from stress-induced ROS overproduction. Antioxidants are molecules that donate an electron to a free radical to neutralize it so that it is no longer as damaging to cellular macromolecules. Antioxidants can be enzymatic or non-enzymatic in nature, and can react with ROS as radical scavengers, hydrogen or electron donors, peroxide decomposers, and singlet oxygen quenchers, among others (Lobo et al., 2010). Many of these antioxidants are produced endogenously through normal metabolic processes, while others are introduced through the diet. Some of the most common antioxidants include glutathione, ubiquinol, uric acid, vitamin $\mathrm{E}$ ( $\alpha$-tocopherol), vitamin $\mathrm{C}$ (ascorbic acid), and $\beta$-carotene (Lobo et al., 2010).

Due to their regular exposure to hypoxic/anoxic conditions and the sudden reintroduction of oxygen that follows, red-eared slider turtles have developed strong antioxidant defences (Storey, 2007). These defences include constitutively high antioxidant activity, as well as inducible defences. For example, the activities of various antioxidant enzymes such as catalase, superoxide dismutase (SOD), and alkyl hydroperoxide reductase are significantly higher in turtles than in other ectothermic vertebrates (Willmore \& Storey, 1997a). Additionally, increased organ pools of glutathione and ascorbic acid, limited succinate accumulation and low succinate/fumarate ratios, and the synthesis of ATP from ADP all contribute to limiting ROS production during reoxygenation and/or coping with any ROS that are produced (Bundgaard et al., 2019; Rice et al., 1995; Willmore \& Storey, 1997b). Furthermore, there is evidence for the increased affinity of antioxidant enzymes for their substrates, as well as the ability to function across a wide $\mathrm{pH}$ range, thus conferring higher efficiency for the reduction of oxidized substrates 
(Willmore \& Storey, 2007). Beyond the constitutive defences, studies have also shown increased levels of inducible antioxidant proteins including the transcription factor Nrf2, heme oxygenase-1 (HO-1), manganese and copper/zinc SOD, various glutathione Stransferase (GST) isozymes, and ferritin heavy chain (Breedon et al., 2021; Hermes-Lima \& Zenteno-Savín, 2002; Kesaraju et al., 2009; Krivoruchko \& Storey, 2010c). These adaptations all lend towards red-eared slider turtles having much stronger antioxidant defences than other non-anoxia-tolerant animals, making them able to overcome the challenges ROS imposes during frequent anoxia-reoxygenation cycles; in fact, studies have shown that these turtles suffer little to no oxidative stress during reoxygenation events (Milton et al., 2007; Willmore \& Storey, 1997a, 1997b).

Another important molecular safeguard during anoxia-reoxygenation cycles are the various chaperone proteins, which stabilize the structure of proteins that are sensitive to stress-induced changes in intracellular conditions. Alterations to the intracellular environment can cause proteins to misfold from their native conformations. Heat shock proteins (HSPs) are one of the best understood stress-responsive cytoprotective mechanisms, working to fold nascent or misfolded proteins into their proper structure so they are able to function normally (Tutar \& Tutar, 2010). The action of HSPs and other chaperones are critical to preserving and extending the lifespan of cellular proteins, which is important during times of stress when intracellular conditions are altered, and the translation of new proteins is halted to enter a hypometabolic state (discussed in more detail in Section 1.3.2.). Studies have shown that red-eared slider turtles display high constitutive HSP expression, as well as stress-induced increases to HSP levels (Kesaraju et al., 2014; Krivoruchko \& Storey, 2010d; Prentice et al., 2004; Stecyk et al., 2012). Additionally, 
other chaperone proteins including aB-crystallin, GRP78, and GRP94 have also been shown to increase during anoxic stress (Krivoruchko \& Storey, 2013; Storey, 2007). Activation of HSPs and other chaperones likely serves to maintain protein stability under changing intracellular conditions during anoxia and to prepare the cell for reoxygenation where protein damage by oxidative stress would otherwise occur.

Without robust cellular defence and pro-survival processes, the red-eared slider turtle would be vulnerable to a litany of adverse side effects brought about by limited oxygen availability. These defence mechanisms, along with various other smaller pathways, work in tandem to protect the red-eared slider turtle from the deleterious effects of anoxia and reoxygenation. However, one of the most critical adaptations to survive anoxia is the ability to massively reduce the amount of cellular energy consumed during the stress period through the entrance into a hypometabolic state and the reprioritization of energy expenditure.

\subsubsection{Hypometabolism and metabolic reorganization}

There are two key metabolic adaptations that allow turtles to survive extended anoxic episodes: (1) metabolic rate depression (MRD) to slow substrate depletion and metabolic by-product accumulation, and (2) reorganizing energy usage to maintain balanced cellular energetics (i.e. matching the rate of energy production and energy consumption). The aforementioned cellular defence and pro-survival mechanisms (Section 1.3.1.) work in tandem with MRD to protect turtles from extensive stress-related damage. Turtles are particularly adept at achieving a state of hypometabolism during winter hibernation, due in part to their relatively low normoxic metabolic rate; these ectotherms have low metabolic rates at any temperature when compared to that of an endotherm 
(Jackson \& Ultsch, 2010). Furthermore, because they are ectotherms, lowering the environmental temperature causes a matching decrease in body temperature, which decreases their metabolic rate through the slowing of chemical reactions (Jackson \& Ultsch, 2010). Additionally, prolonged underwater submergence further reduces the turtle's metabolic rate (Jackson, 1968; Jackson \& Ultsch, 2010). Overall, these mechanisms can lead to a reduction in metabolic rate by around $90 \%$ compared to their resting normoxic rate (Hochachka, 1988; Jackson, 1968; Storey, 2007). This metabolic suppression reduces the cellular demand for ATP to an extent that it can be met by the less-efficient anaerobic metabolism, allowing turtles to survive extended periods of anoxia without suffering lasting damage (Storey \& Storey, 2004). Turtles are able to fuel this reduced metabolic need during aphagic hibernation using relatively large organ glycogen stores, particularly in the liver (Jackson \& Ultsch, 2010). These glycogen reserves are typically sufficient enough to support aerobic or anaerobic metabolism for as long or longer than the turtle would naturally experience during over-winter underwater submergence, meaning that glycogen depletion would not usually pose a threat to their long-term survival (Jackson \& Ultsch, 2010).

With the entrance into hypometabolism and the anoxia-associated reduction in ATP production, there is a similar decrease in energy consumption facilitated by the reorganization of metabolic priorities for ATP expenditure (Krivoruchko \& Storey, 2015; Storey, 2007). If this reorganization does not occur, anaerobic metabolism will not be sufficient to meet the energetic demands of the cell, which will eventually lead to death of the organism (Krivoruchko \& Storey, 2015; Storey, 2007). During brief exposures to low oxygen conditions, anaerobic glycolysis is typically sufficient to meet the short-term 
energetic requirements for keeping an animal alive with normal physiological functioning. However, the long-term viability of the turtle is ensured by the extrapulmonary uptake of oxygen, and the halting of energetically expensive processes (Storey, 2007). Most turtles have some capacity to survive underwater without pulmonary respiration via the exchange of gases across the epithelium lining of the skin, buccopharyngeal membrane, or the cloaca/cloacal bursae (Jackson \& Ultsch, 2010; Ultsch, 2006). The extent to which different turtle species use extrapulmonary oxygen uptake varies, although in North American species, the rate of gas exchange is typically insufficient to survive underwater at summer temperatures (Belkin, 1968; Jackson et al., 2004; Jackson \& Ultsch, 2010). However, at winter temperatures when the turtle's metabolic rate is suppressed, the demand for oxygen is lessened to such an extent that they are able to obtain enough oxygen from aerated water to meet most, if not all, of their metabolic requirements (Jackson \& Ultsch, 2010). This minimizes their reliance on anaerobic metabolism, meaning that, so long as the water remains aerated, aerobic respiration is able to continue; however, during monthslong submergence in ice-covered bodies of water, oxygen typically becomes rapidly depleted (Krivoruchko \& Storey, 2015).

Whilst in prolonged submergence, once the turtle's blood oxygen levels drop below arterial $\mathrm{pO}_{2}$ values of approximately 20 Torr, oxidative phosphorylation halts, and anaerobic glycolysis is used as the main ATP source (Hochachka, 1988; Jackson, 1968; Storey \& Storey, 1990, 2004). As described in Section 1.1.3., this anaerobic pathway produces significantly less ATP than aerobic respiration; hence the need for molecular controls that suppress energetically expensive cell functions (Biggar \& Storey, 2011; Storey \& Storey, 2004). These regulatory mechanisms work to reprioritize energy usage 
towards critical stress-responsive gene expression over genes associated with non-critical pathways. Many cellular processes that are important for normal cell function (e.g. protein synthesis, protein degradation, apoptosis, cell proliferation and cell cycle progression) do not aid in stress-survival and are incredibly energetically expensive, making them key targets for suppression by various means. In the red-eared slider turtle, each MRD mechanism plays a part in the much larger summative goal of global transcriptional and translational repression of non-critical pathways, while allowing for the expression of anoxia-responsive genes.

The regulation of transcription results from the combined effects of the structural conformation of DNA and transcription factors. The structural properties of DNA-what is commonly referred to as its packaging - is integral to how that DNA is transcribed. Many epigenetic modifications can be used to alter gene expression during hypometabolism. Briefly, in eukaryotic organisms, DNA is complexed with histones to form nucleosomes, which collectively form chromatin (Annunziato, 2008). In order for transcription to proceed, the chromatin must be unwound, while modifications associated with closed chromatin structures lead to decreased transcription. Changes to chromatin state are coordinated mainly by reversible DNA methylation and histone PTMs (e.g. acetylation, methylation, and phosphorylation) in response to environmental stimuli (Morin \& Storey, 2009; Storey, 2015). These epigenetic mechanisms support global gene silencing and the reprioritization of energy usage by constricting which sections of DNA are readily available for transcription factors and RNA polymerase binding, thus blocking transcription (Storey, 2015). Typically, DNA methylation and amino acid-specific histone methylation, deacetylation, and phosphorylation all serve to silence transcription (Allis \& 
Jenuwein, 2016; Jaenisch \& Bird, 2003). In the red-eared slider turtle, a study has shown a tissue-specific increase in DNMT expression and activity along with increased overall DNA methylation during anoxia, altogether correlating to decreased gene expression (Wijenayake \& Storey, 2016). Additional studies have similarly found a tissue-specific downregulation of HAT-mediated histone acetylation and an upregulation of certain HDACs responsible for histone deacetylation, with an overall decrease in histone acetylation noted (Krivoruchko \& Storey, 2010a; Wijenayake \& Storey, 2020). Research into histone methylation has found contradictory expression of methylation marks associated with active and repressed transcription as well as KMTs, potentially pointing towards dynamic regulation leading to global transcription suppression with selective upregulation of anoxia survival genes (Wijenayake et al., 2018). Overall, these findings point to the role of epigenetics in achieving global gene suppression to conserve energy during anoxia.

Alongside the downregulation of non-necessary gene transcription, anoxiaresponsive transcription factors can upregulate the expression of crucial protective mechanisms and pro-survival pathways necessary to anoxia survival. Additionally, the limited amount of ATP produced is reprioritized to be spent only on transcription of the most essential genes. One of the main mechanisms this is achieved is through stressresponsive transcription factors. These regulatory proteins are responsible for the activation of transcription through DNA-binding and are a common mechanism for the activation of pro-survival pathways. Anoxia-responsive transcription factors control which genes are expressed and to what extent during low oxygen conditions. One of the hallmark transcription factors associated with oxygen limitation is hypoxia inducible factor-1 (HIF- 
1) (Adams et al., 2009; Ziello et al., 2007). HIF-1 is an oxygen-sensing transcription factor that rapidly responds to oxygen limitations in various ways including upregulating the expression of genes associated with improved delivery of oxygen to tissues around the body, and the enhancement of anaerobic glycolytic capacity (Biggar \& Storey, 2011; Morin \& Storey, 2005). The HIF-1 heterodimer is composed of two subunits, HIF-1 $\alpha$ and HIF$1 \beta$, of which HIF- $1 \alpha$ contains an oxygen-sensitive region, oxygen-dependent degradation domain (ODDD) (Adams et al., 2009; Ziello et al., 2007). Under normoxic conditions, oxygen regulates ODDD, ultimately leading to its degradation (Semenza, 2007). This regulation means that only under hypoxic or anoxic conditions can the two subunits dimerize to activate the transcription of genes critical for anaerobic glycolysis such as pyruvate kinase, lactate dehydrogenase, phosphofructokinase, and pyruvate dehydrogenase kinase (Semenza, 2007). As such, HIF-1 and other anoxia-induced transcription factors are critical for anoxia-responsive gene expression and the long-term survival of red-eared slider turtles during hibernation.

Moreover, the proteins that are produced are a site of regulation themselves. Given how much energy translation requires, it is useful for organisms to halt protein turnover. This means not only slowing or stopping the production of new proteins, but also stabilizing existing proteins to prevent their degradation. This is done primarily through the use of chaperone proteins as previously mentioned in Section 1.3.1. Stabilizing the already existing proteins and extending their lifespan negates the need for new proteins to translated, thus saving the energy required for continual production, which can be used for other cellular processes. In fact, over $50 \%$ of ATP was used for protein turnover in turtle 
hepatocytes under normoxic conditions but was reduced by $90 \%$ during anoxia (Hochachka \& Lutz, 2001).

Protein synthesis requires a significant amount of ATP, and while this energetic need is tolerated under normoxic conditions, when red-eared slider turtles undergo annual winter dormancy they have limited energy production and cannot afford this energy cost. Indeed, previous studies have shown a drop in protein synthesis related ATP usage during anoxia to approximately $6 \%$ of normoxic values (Hochachka et al., 1996). Studies have shown a strong reduction in the rate of protein synthesis after prolonged anoxia in turtles, with the rates rapidly returning to normoxic values after aerobic recovery (Bailey \& Driedzic, 1996; Brooks \& Storey, 1993; Fraser et al., 2001; Land et al., 1993). Though there is decreased protein production, total mRNA transcripts levels of most constitutively expressed genes did not significantly decrease in anoxic turtles (Douglas et al., 1994; Land et al., 1993). This discrepancy can be accounted for by post-transcriptional inhibition of protein synthesis. One key mechanism for this is reversible mRNA silencing via microRNA (miRNA) binding. miRNA are a powerful tool for the dynamic regulation of translation, especially in response to environmental stress. The background and function of miRNA during stress-response is explained in more detail in Section 1.4. Though there is much evidence for its use in achieving MRD and metabolic reorganization in many stress-tolerant species, there is little work done to date on the red-eared slider (Biggar \& Storey, 2018a; Hadj-Moussa \& Storey, 2020; Storey, 2015). However, given their implication in stress survival and MRD due to their ability to quickly achieve mRNA silencing and the reverse it just as quickly upon reoxygenation, miRNA is a critical aspect 
of the red-eared slider turtle's anoxia tolerance with effects far beyond the few miRNA species that have been selectively studied so far.

\subsection{Post-transcriptional regulation of gene expression via microRNA}

miRNA are short ( $22 \mathrm{nt})$, non-coding RNA molecules that bind complementary target mRNA transcripts to repress their translation (see Section 1.4.2.) (Finnegan \& Pasquinelli, 2013). These essential RNA species are one facet of the intrinsic RNA interference (RNAi) pathway, which, along with small interfering RNA (siRNA), are produced from double-stranded RNA (dsRNA) and used for targeted gene silencing. First discovered in 1993 in Caenorhabditis elegans, the uncovering of lin-4 and let-7 marked the beginning of an ever-expanding field of miRNA-mediated gene regulation (Lee et al., 1993; Paciello et al., 2011; Reinhart et al., 2000). Since then, miRNAs have experienced an influx of research implicating them in diseases, and disorders such as various cancers, organ disease including liver and heart, and neurological disorders including Alzheimer's and schizophrenia (Ardekani \& Naeini, 2010; Walayat et al., 2019).

miRNAs have also been implicated in the natural stress-tolerance of numerous organisms as a critical aspect of MRD (Storey, 2015). Regulation via miRNA allows an organism to rapidly and reversibly suppress mRNA transcript expression in response to changing environmental conditions in a tissue-specific manner. miRNAs target the majority of all mammalian mRNAs and likely have similar coverage in all other animals, giving them control of a wide breadth of genes and their associated cellular processes (Storey, 2015). This is particularly useful during periods of anoxia because without access to oxygen, ATP synthesis is massively impeded, and translation of mRNAs into proteins is extremely energetically expensive. The use of miRNA to facilitate MRD is highly 
conserved across phylogeny. For example, miRNA regulation has been observed in numerous MRD animal models including: the freeze-tolerant frog (Rana sylvatica); estivated African clawed frog (Xenopus laevis); hibernating gray mouse lemurs (Microcebus murinus), brown bears (Ursus arctos) and thirteen-lined ground squirrels (Ictidomys tridecemlineatus); and hypoxic crayfish (Orconectes virilis) and naked-mole rats (Heterocephalus glaber), among others (English et al., 2018; Hadj-Moussa et al., 2020, 2021; Hadj-Moussa \& Storey, 2018; Hawkins \& Storey, 2020; Logan \& Storey, 2021; Luu et al., 2020).

Given just how widespread the effects of miRNA are, it follows that they would also be critical to the regulation of protein translation in anoxic red-eared slider turtles. In fact, a recent study has measured the expression of 11 miRNAs in anoxic red-eared slider turtles and found the miRNA are differentially expressed during anoxia, in a tissue-specific manner (Biggar \& Storey, 2017). They also found that the miRNA tended to target processes associated with metabolism and cell proliferation (Biggar \& Storey, 2017). This finding is supported by a previous study that analyzed cyclin D1 levels and found a general repression of cell proliferation during anoxia (Biggar \& Storey, 2012). This study also analyzed the expression of two miRNAs that target cyclin D1 and found both increased in liver and kidney, but not in muscle (Biggar \& Storey, 2012). These studies show that miRNA is a critical regulator of cell cycle progression and cell proliferation in the redeared slider turtle during anoxic stress. Despite their criticality, the complete miRNA expression profile of the anoxic red-eared slider turtle has never been analyzed in full. Additionally, no previous research has provided an in-depth look at the regulation of miRNA biogenesis in turtles, despite this being a key regulatory step affecting miRNA 
expression, and thus, gene expression as a whole. Hence, the focus of this thesis is to analyze the regulation of miRNA biogenesis and its downstream effect on mRNA fate, and to characterize the complete anoxic miRNA profile in the red-eared slider turtle.

\subsubsection{MicroRNA biogenesis and maturation}

The canonical production of miRNA (Figure 1.3) begins in the nucleus with the transcription of a non-protein-coding miRNA gene by RNA polymerase II (Kim, 2005). This miRNA transcript forms the double-stranded primary miRNA (pri-miRNA) (Kim, 2005). Next, the 5' and 3' trailing ends are cleaved from the pri-miRNA by the microprocessor complex, leaving behind the double-stranded hairpin loop structure of the precursor-miRNA (pre-miRNA) (Kim, 2005). The microprocessor complex is formed primarily by the joining of Drosha and two DiGeorge syndrome critical region 8 (DGCR8) proteins (Kim, 2005). Drosha is a class 2 ribonuclease (RNase) enzyme, containing two RNase III domains, as well as a dsRNA-binding domain that, along with DGCR8, allows the microprocessor complex to bind to the double-stranded pri-miRNA so that it can remove its 5' and 3' trailing ends (Finnegan \& Pasquinelli, 2013; Kim, 2005). DGCR8, also known as Pasha, is the non-catalytic subunit of the microprocessor complex, which aids in pri-miRNA binding via its two dsRNA-binding domains to help facilitate cleavage by Drosha (Filippov et al., 2000; Kim, 2005; Wu et al., 2000). These proteins complex to form the microprocessor whose function is autoregulated through the protein-protein interactions of Drosha and DGCR8 (Finnegan \& Pasquinelli, 2013; Yeom et al., 2006). Microprocessor expression is autoregulated through Drosha and DGCR8 posttranscriptionally regulating each other (Finnegan \& Pasquinelli, 2013). The 5'-UTR of DGCR8 mRNA transcripts contain a hairpin loop that resembles pri-miRNA, which leads 
to microprocessor bindings and cleavage of the DGCR8 transcript, while DGCR8 stabilizes Drosha (Finnegan \& Pasquinelli, 2013). This autoregulation serves to maintain consistent miRNA biogenesis and expression (Han et al., 2009; Kadener et al., 2009; Maclas et al., 2012).

Following cleavage of the 5' and 3' trailing ends, the pre-miRNA is exported from the nucleus into the cytoplasm via Ran-GTP dependent exportin-5 (XPO5) binding (Finnegan \& Pasquinelli, 2013). XPO5 binds to the pre-miRNAs along with its cofactor Ran-GTP — stabilizing and protecting the pre-miRNA from exonuclease degradation in the process - until it is exported into the cytoplasm via nuclear transport receptors (Y. Lee et al., 2002). Once in the cytoplasm, the hydrolysis of GTP to GDP causes the pre-miRNA to be released where it can be further processed (Finnegan \& Pasquinelli, 2013). Next, Dicer mediates the cleavage of the pre-miRNA hairpin loop structure into an approximately $21-$ 24 nt mature miRNA duplex, alongside its cofactors, TAR RNA-binding protein (TRBP) and protein activator of PKR (PACT) (Lei \& Silver, 2002). Dicer is an exonuclease that contains a helicase, PAZ (an RNA-binding motif that recognizes 3' overhangs of miRNA duplexes), dsRNA-binding, and two RNase III domains (Finnegan \& Pasquinelli, 2013). TRBP stabilizes and stimulates Dicer to increase the efficiency at which it can cleave premiRNA (Finnegan \& Pasquinelli, 2013; MacRae \& Doudna, 2007). PACT is a dsRNAbinding protein that associates with Dicer and TRBP, potentially working with TRBP to achieve the synchronization of dual pre-miRNA cleavage with Dicer's two RNase III domains (Chakravarthy et al., 2010; Finnegan \& Pasquinelli, 2013; Koscianska et al., 2011). 
Following cleavage, the miRNA duplex is deposited into the RNA induced silencing complex (RISC)-loading complex (RLC), which consists of Dicer, TRBP, and Argonaute (AGO) proteins (Finnegan \& Pasquinelli, 2013; Koscianska et al., 2011). The presence of AGO frees Dicer from the mature duplex so that it can continue cleaving new pre-miRNA (Finnegan \& Pasquinelli, 2013; O’Brien et al., 2018). Additionally, AGO2 contains endonuclease activity to cleave the miRNA, which may aid in strand selection; however, some AGO proteins do not contain endonuclease activity, hence, this activity is not necessary for all miRNA processing (Finnegan \& Pasquinelli, 2013). Next, one strand of the duplex (the guide/template strand) is loaded onto AGO to form the miRISC (Finnegan \& Pasquinelli, 2013). The guide strand is used by AGO to target the complementary mRNA in order to repress their translation (Finnegan \& Pasquinelli, 2013; O'Brien et al., 2018). The other miRNA strand, termed the passenger strand (miRNA*) is degraded, however, it may occasionally also be loaded onto AGO as part of the miRISC (Finnegan \& Pasquinelli, 2013; Huntzinger \& Izaurralde, 2011). Strand selection seemingly relies mostly on the stability of the 5 ' terminus, with less stable strands more likely to be used as the guide (Okamura et al., 2008). Some AGOs contain catalytic activity making them able to cleave the mRNA if pairing is perfectly complementary, but perfect pairing is relatively rare in animals (Finnegan \& Pasquinelli, 2013; Khvorova et al., 2003). Instead, AGOs typically interact with cofactors to target the mRNA for deadenylation and subsequent degradation (Finnegan \& Pasquinelli, 2013).

There are also multiple other non-canonical miRNA biogenesis pathways that use different combinations of the aforementioned canonical proteins (Finnegan \& Pasquinelli, 2013; Huntzinger \& Izaurralde, 2011). These pathways are typically either 
Drosha/DGCR8-independent, or Dicer-independent (O’Brien et al., 2018). For example, mirtrons produced from mRNA introns during splicing are similar enough to pre-miRNA that Dicer can immediately cleave them, skipping pri-miRNA cleavage by Drosha and DGCR8 (O’Brien et al., 2018). Additionally, short hairpin RNAs (shRNAs) are cleaved by Drosha, but are too short to be recognized by Dicer once in the cytoplasm so they are directly loaded onto AGO2, which cleaves the 5' end (O'Brien et al., 2018; Ruby et al., 2007). Altogether, these alternative miRNA biogenesis pathways generally have the same outcome as the canonical pathway, in that they all produce miRNAs that can functionally silence mRNA translation through their decay or inhibition.

\subsubsection{MicroRNA-mediated messenger RNA silencing}

Once a miRNA is fully matured, it directs the RISC complex to its target mRNA. Each individual miRNA can bind to multiple separate mRNA transcripts, and each mRNA can be targeted by multiple miRNAs (O'Brien et al., 2018; Yang et al., 2010). This combinatory effect provides fine control over the translation and fate and mRNA. miRNA work by binding to the 3'-UTR of mRNA transcripts and, depending on the complementarity of their seed sequence to the mRNA target, can either target mRNA for degradation or for temporary silencing (Bartel, 2004). The seed sequence is the 5' region of the mature miRNA guide strand consisting of nucleotides $2-8$, which target complementary motifs in the 3'-UTR of mRNAs (O'Brien et al., 2018). As seen in Figure 1.4, the miRNA guide strand targets RISC activity towards the appropriate mRNA, ultimately leading to mRNA silencing through decay or inhibition (O'Brien et al., 2018). Perfect complementary miRNA:mRNA binding leads to the degradation of the bound mRNA via AGO endonucleases, however, perfect binding is relatively rare in animals 
(Bartel, 2004). Imperfect complementarity of miRNA:mRNA binding leads to translational suppression, with the mRNA transcript being shuttled into cytoplasmic foci associated with mRNA decays and/or sequestration until the stress event has abated and the cellular conditions support translation once again (see Section 1.5.) (Bartel, 2004; Finnegan \& Pasquinelli, 2013; He \& Hannon, 2004). The decay of mRNA is facilitated by a miRISC cofactor, GW182, which helps recruit poly(A)-deadenylase complexes to the miRNAbound mRNA (O'Brien et al., 2018). Poly(A)-binding protein C (PABPC) helps achieve efficient deadenylation, after which, the mRNA is decapped by various proteins and degraded by exoribonuclease 1 (XRN1) (Christie et al., 2013; Jonas \& Izaurralde, 2015; O'Brien et al., 2018). Additionally, translational inhibition can be achieved through the stalling of the $48 \mathrm{~S}$ pre-initiation complex on the 5' UTR of the mRNA, which blocks further ribosomal binding (Jonas \& Izaurralde, 2015; O’Brien et al., 2018).

This mechanism for mRNA silencing is critically important to the routine functioning of the cell as is evidenced by high miRNA conservation between distantly related species. Moreover, the role miRNA plays in stress responsive MRD and gene regulation cannot be underscored. These miRNA species provide rapid and reversible control over a broad range of cellular processes, which enables organisms such as the redeared slider turtle to quickly suppress their metabolic rate to such an extent that they are able to survive prolonged anoxic episodes. Their dynamic characteristics make them ideal for imparting the animal-, tissue-, and stress-specific regulation of gene expression that is necessary for successful stress tolerance. 


\subsection{Stress granules, processing bodies, and mRNA fate following microRNA binding}

A variety of non-membranous cellular sub-compartments called messenger ribonucleoprotein (mRNP) granules exist the cytosol, including stress granules, processing bodies (P-bodies), among others (Anderson \& Kedersha, 2008). Stress granules and Pbodies both participate in mRNA silencing because they both serve as sites for the storage of translationally repressed mRNAs, and P-bodies also participate in mRNA decay (Standart \& Weil, 2018). The packaging of mRNA into these granules regulates gene expression through translational inhibition of specific transcripts.

\subsubsection{Stress granules as sites of $m R N A$ storage}

Stress granules are cytosolic nucleoprotein aggregates that are formed in response to environmental stresses such as low oxygen, high temperatures, oxidative stress, viral infections, osmotic shock, nutrient starvation, and proteotoxic stress, and act to transiently store mRNAs during these stress conditions (Standart \& Weil, 2018). The sequestering of mRNA prevents their translation in order to conserve energy during stress events and to prevent the generation of abnormal proteins (Attwood et al., 2020; Kedersha \& Anderson, 2007; Spriggs et al., 2010). This enables the cell to use the limited amount of ATP it has available to prioritize the translation of proteins that are critical to the stress-response, and thus, organismal survival. Stalled transcripts can then undergo translation upon recovery from the stress condition, or they may undergo degradation in P-bodies (see Section 1.5.2.) (Standart \& Weil, 2018).Though the particular mRNA and protein composition of stress granules varies in a stress- and tissue-specific manner, some proteins remain as the core components of stress granules (Parker \& Sheth, 2007). These proteins serve as marker proteins that are not found in other mRNPs (Anderson \& Kedersha, 2009; Buchan \& 
Parker, 2009; Kedersha et al., 2005). Some of these proteins include small ribosomal subunits, eukaryotic translation initiation factors (eIFs), and PABPC1 (Buchan \& Parker, 2009; Kedersha et al., 2005). Additionally, stress granules contain RNA-binding proteins (RBPs), which promote stress granule assembly when highly expressed, including Ras GTPase Activating Protein (SH3 Domain) Binding Protein 1 (G3BP1), T-cell-restricted intracellular antigen-1 (TIA-1), and TIA-related protein (TIAR) (Kedersha \& Anderson, 2007). Furthermore, it has been seen that during certain types of stress, the miRNA silencing machinery RISC localizes to stress granules (Gilks et al., 2004; Kedersha et al., 1999; Tourrière et al., 2003).

Stress granules form via either an eIF2-dependent or -independent pathway. In the eIF2-dependent pathway, kinases act as environmental sensors and phosphorylate eIF2 $\alpha$, which inhibits translation initiation (Anderson \& Kedersha, 2008). In the eIF2-independent pathway, various chemicals can act as inhibitors of particular steps of translation initiation, resulting in the formation of stress granules (Anderson \& Kedersha, 2008; Buchan \& Parker, 2009). In both the dependent and independent pathways, ribosomes fall off the mRNA transcripts as the next round of translation cannot begin whilst initiation is stalled. However, the $48 \mathrm{~S}$ pre-initiation complex remains bound to the 5' UTR of the mRNA, where it likely joins with aggregation-prone proteins (G3BP1, TIA-1, TIAR) to form mRNP oligomers (Dang et al., 2006; Mazroui et al., 2006). Protein-protein interactions and crosslinking with PABP-1 then promote the assembly of mature stress granules (Anderson $\&$ Kedersha, 2008). Following sequestration to stress granules, the mRNAs follow one of three fates: (1) the re-initiation of translation, typically for mRNAs encoding pro-survival proteins; (2) translational silencing and transcript storage; or (3) mRNA degradation in P- 
bodies (Anderson \& Kedersha, 2008). Once in recovery from the stress condition, stress granules dissolve to release the sequestered mRNAs and proteins, whilst the large ribosomal subunit binds the $48 \mathrm{~S}$ pre-initiation complex to rapidly restart translation (Anderson \& Kedersha, 2009; Buchan \& Parker, 2009).

\subsubsection{Processing bodies as sites of $m R N A$ storage and decay}

P-bodies, also known as GW-bodies, are highly conserved, constitutively expressed mRNPs that are involved in the storage and decay of silenced mRNA, although storage is quickly emerging as their primary function (Kedersha et al., 2005). Though they are conserved, their number and size are cell- and environment-specific, and they can be further induced during cellular stresses (Hubstenberger et al., 2017; Jakymiw et al., 2007; Standart \& Weil, 2018). In these cytoplasmic foci, untranslated mRNAs are likely targeted to P-bodies for either decay or storage depending on their sequence identity and/or binding proteins (Standart \& Weil, 2018; Teixeira et al., 2005). Similar to stress granules, the sequestration of mRNA prevents their translation, thereby conserving valuable ATP that can be used elsewhere to expression critical stress-responsive genes. Some of the main proteins associated with P-bodies include the 5'-3'exonuclease XRN1, decapping enzymes PAT1B and LSM1-7, deadenylation factors CCR4 and PAN3, miRNA-mediated mRNA silencing factors AGO1-4, GW182 and PAPBC, nonsense-mediated decay factors UPF1 and SMG5/7, and translational repression factors DDX6, 4E-T, and CPEB1, among others (Decker \& Parker, 2012; Eulalio et al., 2007; Ross Buchan, 2014; Standart \& Weil, 2018). Differently to stress granules, the mRNAs in P-bodies are not stalled at the translation initiation step and are therefore not bound to the 40S ribosomal subunit (O'Brien et al., 2018; Standart \& Weil, 2018). 
The formation of P-bodies results from a liquid-liquid phase transition, which allows RNA and proteins to condense into droplets within the cytosol (Standart \& Weil, 2018). Many proteins appear to be involved in P-body formation, which interact with each other and with RNA to form the molecular network underlying these droplets (Standart \& Weil, 2018). In the P-body, the poly(A) tail of the target mRNA must be cropped via deadenylation before the mRNA can be decapped by the Dcp1/Dcp2 complex (Standart \& Weil, 2018). The 3' end of the mRNA contains a heptometric ring of the proteins Lsm17, which surrounds the RNA (Deshmukh et al., 2008; Schwartz \& Parker, 2000). These proteins interact with Pat1, which acts as a scaffold for the decapping complex (Parker, 2012). Various additional proteins contribute to unwinding, scaffolding, decapping, and inhibition of translation initiation (Chowdhury et al., 2007; Parker, 2012). After decapping, the mRNA is degraded from 5' to 3' by the exonuclease XRN1 (Jain \& Parker, 2013; Kedersha \& Anderson, 2007; Sweet et al., 2007; Swisher \& Parker, 2010).

\subsubsection{The interplay between stress granules, processing bodies, and microRNA}

Stress granules and P-bodies are tied both to each other and to the regular functioning of miRNA-mediated gene silencing. Firstly, these mRNP granules are both associated with the packaging of inactivated mRNAs (Kedersha \& Anderson, 2007). Next, like P-bodies, stress granules form in response to stress, although the composition of stress granules may be dependent on the type of stress and their composition may change over its duration (Kedersha \& Anderson, 2007). In both cases, the mRNA contained within stress granules and P-bodies may be returned to normal translation once the stress event ends (Kedersha \& Anderson, 2007; Mollet et al., 2008). Moreover, some evidence suggests that stress granules may actually stem from P-bodies and vice versa, with studies indicating that 
P-body formation occurs before the formation of stress granules and that stress granules initially overlap with pre-existing P-bodies, though this is still poorly understood and up for debate (Standart \& Weil, 2018). However, there is also evidence that both stress granules and P-bodies can also form independently of each other (Buchan et al., 2008; Hoyle et al., 2007). Additional studies have observed stress granules and P-bodies docking, indicating that these mRNPs may be able to shift their components to the other (Buchan et al., 2008; Mollet et al., 2008). Further studies have shown that, in stressed cells, stress granules and P-bodies are often found closely localized to each other (Kedersha et al., 2005; Wilczynska et al., 2005). Overall, the interactions between stress granules and Pbodies suggests an mRNP cycle where the components of both including their associated mRNA and proteins can be exchanged (Kedersha et al., 2005; Wilczynska et al., 2005).

P-bodies differ in that they are constitutively present in the cell, while stress granules form following stress exposure (Buchan \& Parker, 2009; Parker \& Sheth, 2007). These granules also differ in their specific composition, though they both contain stalled mRNA, miRNAs, and AGO proteins (Kedersha et al., 1999; Standart \& Weil, 2018). Additionally, studies have shown that P-bodies contain RNAi machinery including proteins associated with RISC, and many P-body components are required for the proper and efficient functioning of miRNA silencing (Kedersha \& Anderson, 2007). Interestingly, DDX6 and its cofactors may be targeted to particular mRNAs for P-body storage and/or decay by RISC (Behm-Ansmant et al., 2006; Eulalio et al., 2007; Jakymiw et al., 2005; J. Liu, Valencia-Sanchez, et al., 2005). Furthermore, AGO2 phosphorylation enables its interaction with GW182 and DDX6, and promotes its localization to P-bodies (Nishihara et al., 2013; Standart \& Weil, 2018). It is this connection of the end of miRNA-mediated 
mRNA silencing and subsequent mRNA storage/decay in stress granules and P-bodies that makes it of interest to this study. Given the connection between miRNA, stress granules, P-bodies, and environmental stress, it is reasonable to analyze a few select proteins involved in the formation/function of these cytoplasmic foci to provide preliminary insights into how they are change during anoxia and reoxygenation and what effects this might have on mRNA fate.

\subsection{Discovery-driven objectives}

\subsubsection{Objective 1: Examine expression of miRNA biogenesis and cytoplasmic granule} proteins

Previous studies have shown that red-eared slider turtles and other stress-tolerant animals suppress their metabolism through a complex set of regulatory mechanisms in order to survive extended periods of anoxia. miRNAs are a critical component underlying this MRD, allowing for the rapid and reversible regulation of tissue-specific gene expression. The ability to quickly alter protein translation through miRNA:mRNA binding is important to the maintenance of cellular energetics during anoxic stress and to protect the cell from harmful effects and by-products through the reprioritization of pro-survival pathways. miRNA-mediated silencing also implicates stress granules and P-bodies as a significant component of mRNA regulation. Though the in-depth analysis of stress granules and P-bodies is outside the scope of our laboratory, analyzing the relative expression of various key proteins involved in either granule can provide insights into mRNA fate following miRNA silencing. Specifically, the enlargement or increased number of these foci as indicated by increases in protein expression would likely signal that more mRNA is being shuttled for storage and/or decay, which is at least partially due 
to increased miRNA:mRNA binding. Regulating the expression of miRNA and the cytoplasmic foci associated with mRNA silencing and decay is a critical step in the control of hypometabolism. Therefore, it is expected that there will be tissue-specific expression of proteins associated with miRNA biogenesis, and stress granule and P-body formation. Furthermore, it is expected that red muscle will showcase an expression pattern that is consistent with it being more metabolically dormant during anoxia, as compared to liver, which must maintain the performance of metabolically important processes during anoxia and reoxygenation.

Chapter 3 tests this hypothesis by measuring the expression levels of proteins involved in the miRNA biogenesis and maturation pathway to determine how miRNA

\section{Hypothesis 1:}

MicroRNA biogenesis, stress granule, and P-body proteins will be upregulated in the liver and red muscle of red-eared slider turtles compared to normoxic controls, reflecting increased transcriptional silencing in response to anoxia.

expression is regulated in liver and red muscle during anoxia and reoxygenation. Additionally, the expression of protein targets associated with stress granules and P-bodies were analyzed to determine how mRNA translational silencing and/or decay is altered in response to anoxic stress and recovery. Specifically, this objective is addressed by measuring the expression of 13 miRNA biogenesis proteins (Ago1, Ago2, p-AGO2 ${ }^{\mathrm{Ser} 387}$, p-AGO2 ${ }^{\text {Tyr393 }}$, Ago3, Ago4, DGCR8, Dicer, Drosha, PACT, Ran-GTP, TRBP, XPO5), and five stress granule and P-body proteins (GW182, PABPC-1, TIA-1, TIAR, XRN1) via immunoblotting. These results would indicate that miRNA biogenesis and mRNA 
storage/decay are critical points of tissue-specific control for the regulation of miRNA expression in liver and red muscle during anoxia and aerobic recovery.

\subsubsection{Objective 2: Examine miRNA expression and predict corresponding downstream effects}

miRNAs play an important role in the entrance into and maintenance of MRD during periods of environmental stress. Therefore, it is expected that miRNAs will silence mRNA transcripts associated with energetically expensive processes/pathways, allowing only the expression of key proteins necessary for anoxia survival. In contrast to metabolic dormancy of red muscle during anoxic overwintering periods, the liver performs metabolically important processes during anoxia. Particularly, the synthesis of glucose to fuel glycolysis in the absence of food sources during the winter months mainly occurs from liver glycogen stores and is critical to the red-eared slider turtle being able to continually produce ATP. Although most cellular processes will be suppressed to conserve energy during anoxia, certain pro-survival pathways must continue in the liver, demonstrating the need for dynamic regulation of gene expression, which miRNA is especially suited to provide. Hence, it is expected that mRNA transcripts related to pro-survival pathways and processes will remain active, demonstrating the dynamic regulation that occurs as a result of miRNA-mediated gene silencing during anoxia. 


\section{Hypothesis 2:}

Certain microRNAs will be differentially expressed in the liver of red-eared slider turtles during anoxia, and these will contribute to the suppression of energetically expensive cellular processes that are not essential for long-term anoxia survival, such as cell cycle progression.

Chapter 4 tests this hypothesis by analyzing the specific liver miRNA expression profile with the goal of analyzing the differential expression of miRNA in response to anoxia. This objective was addressed by performing miRNA sequencing to determine expression profiles of normoxic control and anoxic turtles, and additional bioinformatic analyses were conducted to predict which downstream cellular pathways and functions were significantly altered during anoxia. The results from these analyses would identify the liver-specific anoxic miRNA expression profile, as well as the processes are likely altered in response to anoxic stress. 


\subsection{FIGURES}

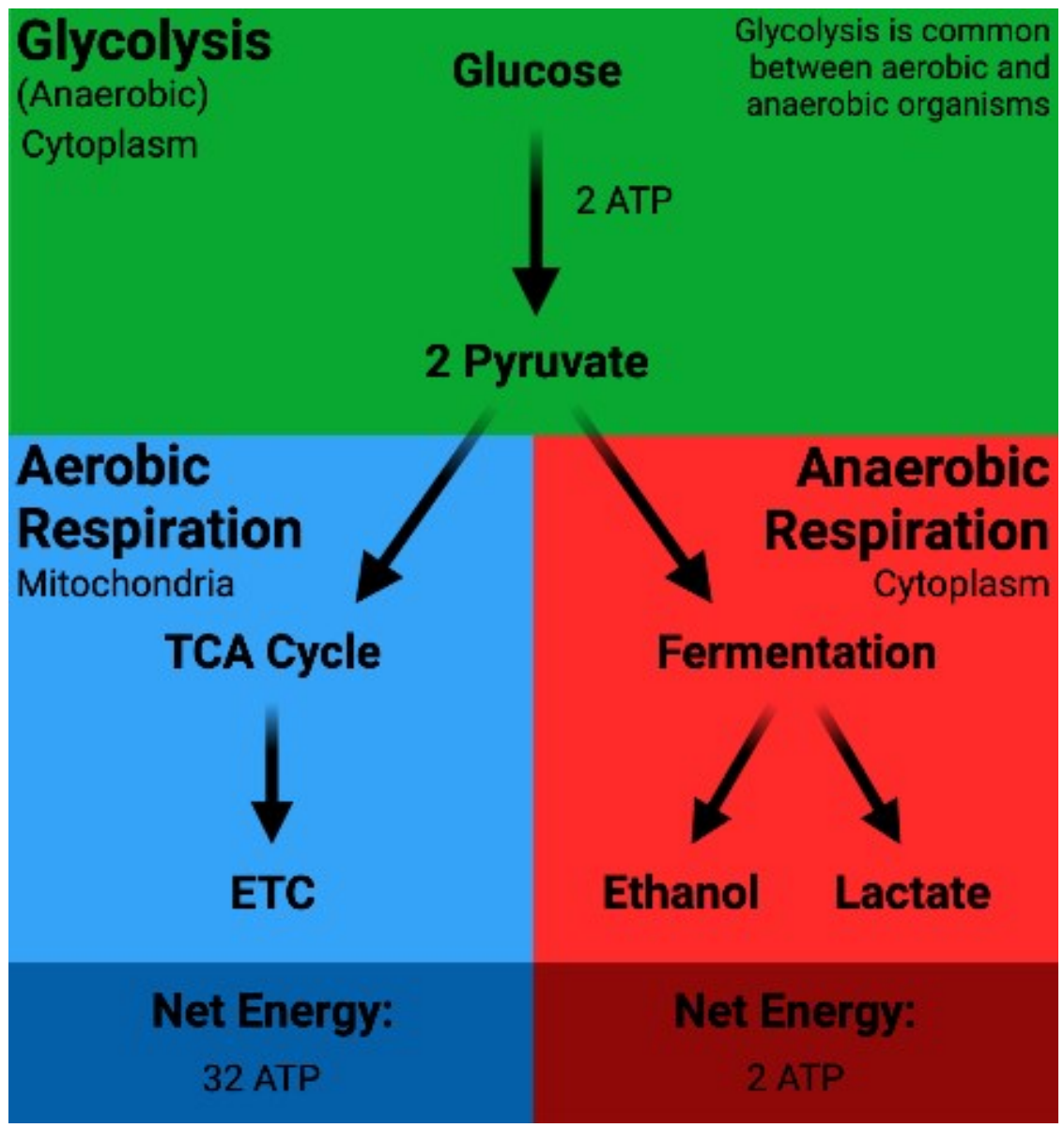

Figure 1.1. General overview of eukaryotic aerobic and anaerobic respiration including net energy production following completion of all processes necessary for energy metabolism. 


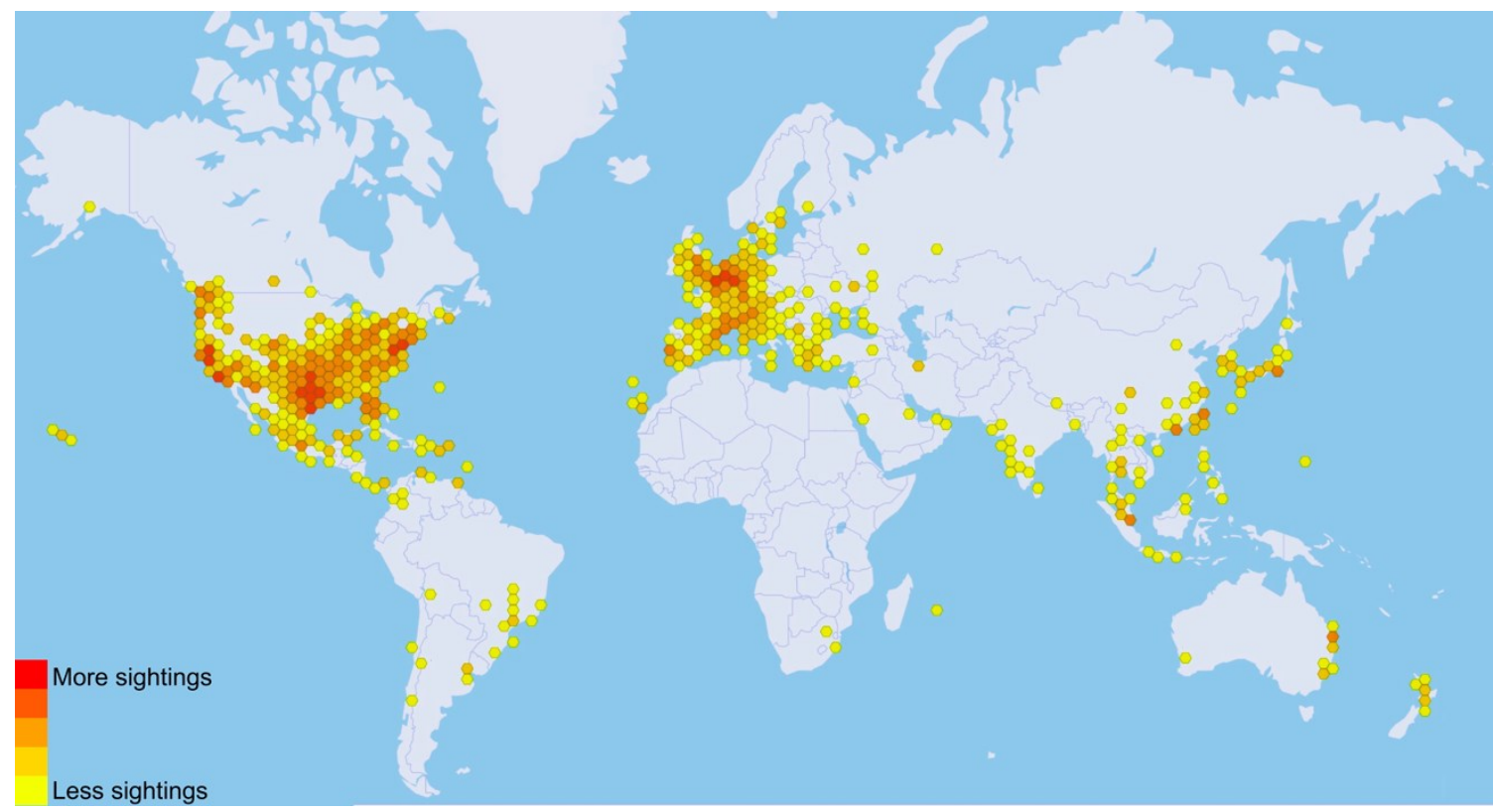

Figure 1.2. Global range of the red-eared slider turtle (Trachemys scripta elegans) as of 2021. Coloured hexagons represent official sightings, with the red to yellow colour scale indicating more to less reports, respectively. Figure adapted from the Global Biodiversity Information Facility website (https://www.gbif.org) (Global Biodiversity Information Facility, 2021). 


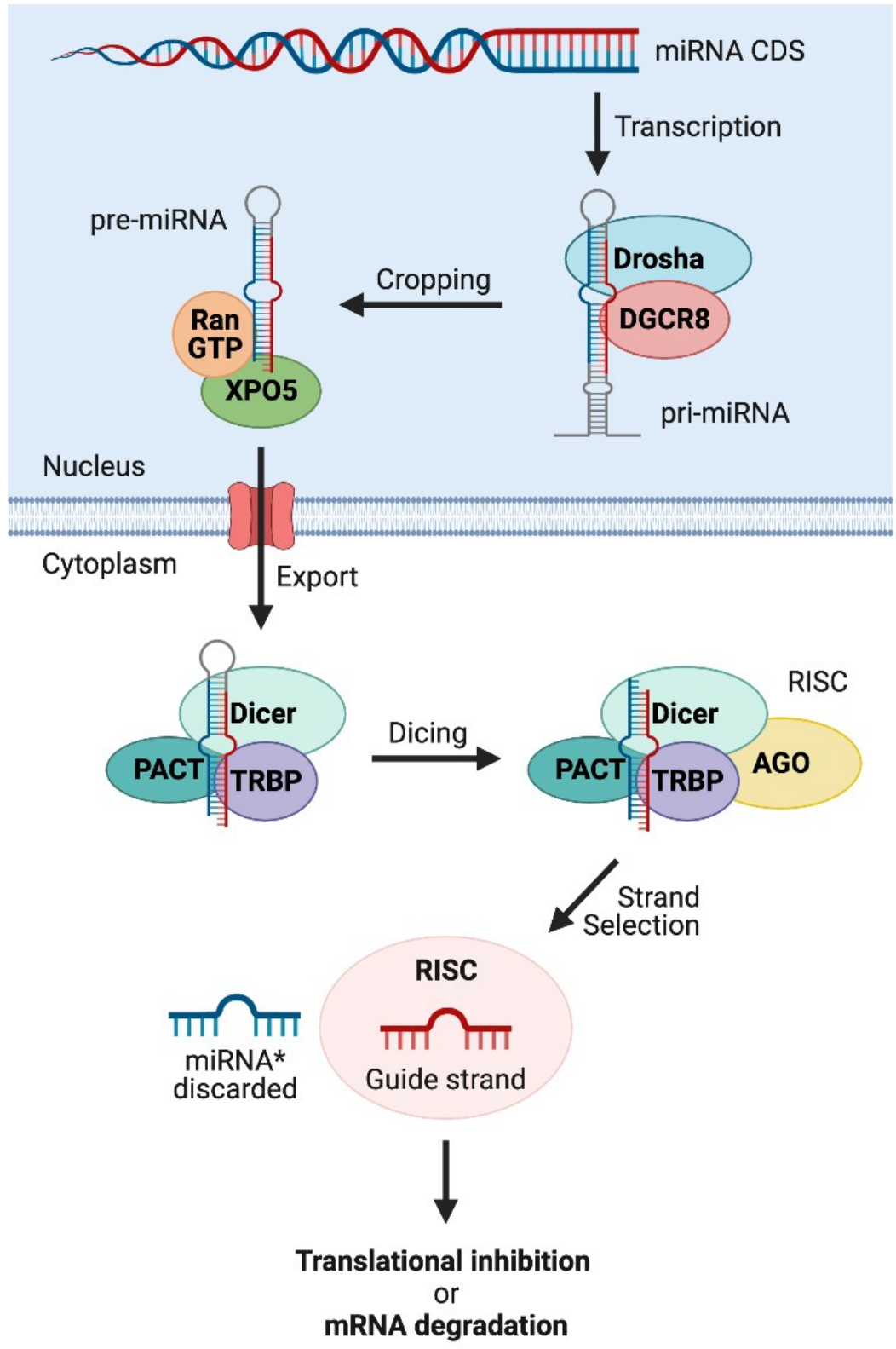

Figure 1.3. Canonical microRNA biogenesis and maturation pathway. Transcription of a miRNA coding sequence (CDS) results in primary miRNA (pri-miRNA) formation. The pri-miRNA is joined by Drosha and DGCR8 to crop the 5' and 3' trailing ends, leaving behind the stem loop structure of the precursor miRNA (pre-miRNA). Pre-miRNA is exported into the cytoplasm via XPO5 and Ran-GTP where it is bound by Dicer, TRBP, 
and PACT. Dicer cleaves the hairpin loop leaving only the miRNA duplex. The duplex is unwound, and the guide strand is loaded onto AGO where it can then be used to target mRNA for translational inhibition or degradation. Figure created using BioRender.com. 


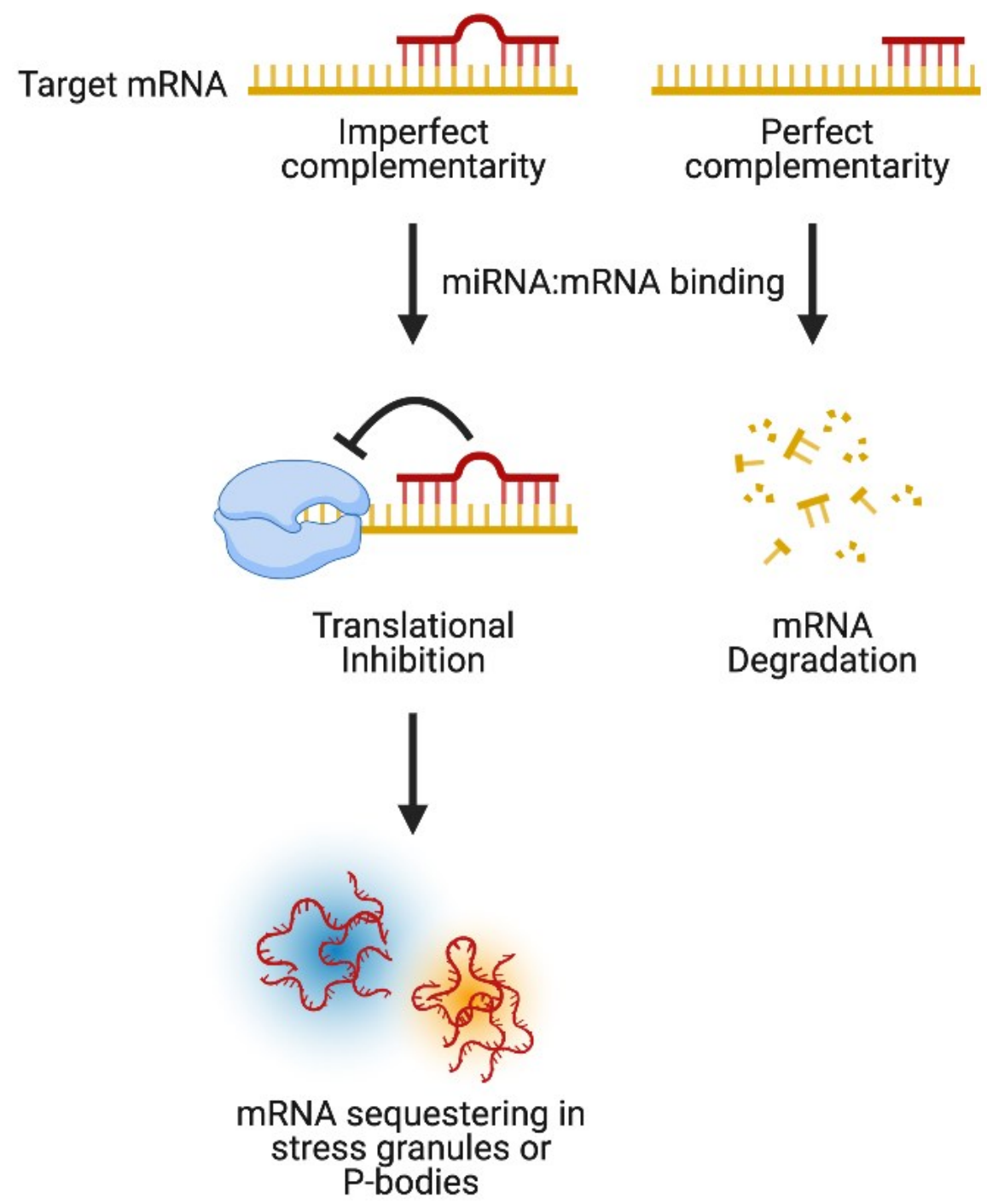

Figure 1.4. MicroRNA targeting of messenger RNA and its translational effects. Perfect complementarity of the miRNA seed sequence to a mRNA transcript induces AGO2dependent mRNA degradation, while imperfect complementarity results in the temporary suppression of translation by mRNA sequestration in stress granules and/or processing bodies. Figure created using BioRender.com. 


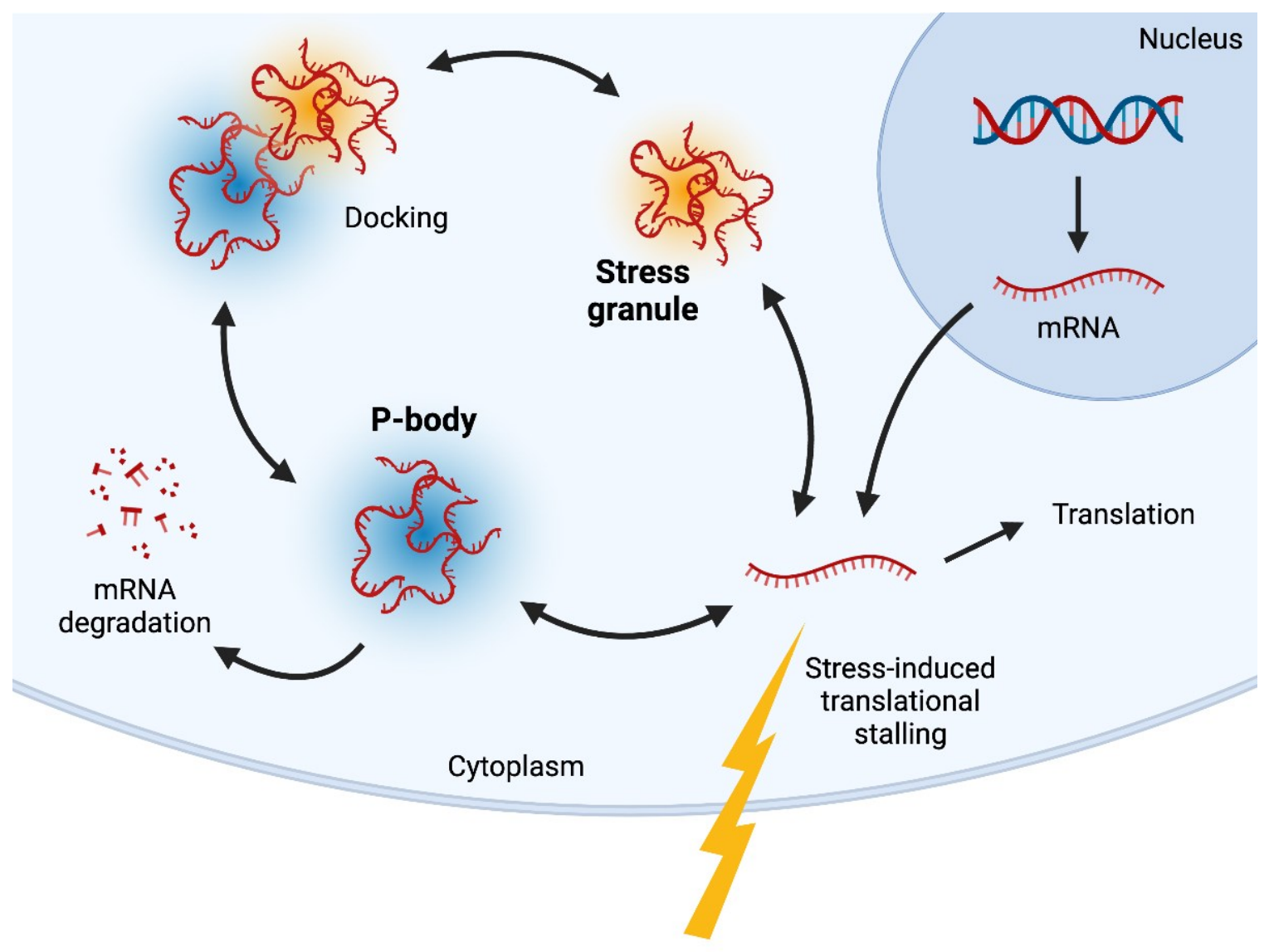

Figure 1.5. Stress granule and processing body sequestration of mRNA in response to stress. Stress-induced translational stalling can lead to the sequestering of affected mRNA transcripts into P-bodies or stress granules. These granules contain silenced mRNA, RNAbinding proteins, and translation initiation factors, which can be exchanged when they dock. The mRNAs contained in P-bodies may undergo degradation in addition to longterm storage. When the stress resolves, mRNA stored within these granules are released to restart translation. Figure is adapted from (Y. R. Li et al., 2013). Figure created using BioRender.com. 


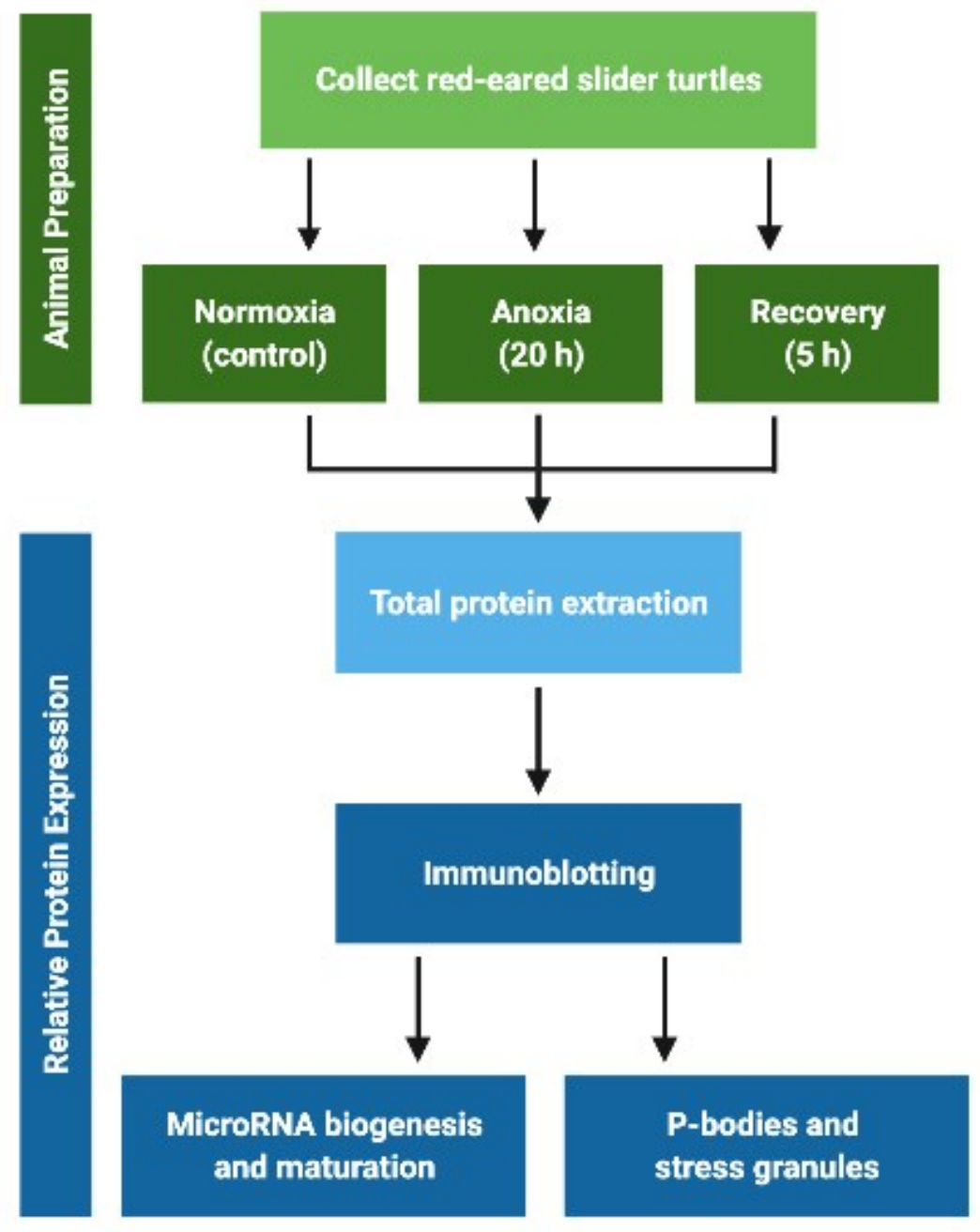

Figure 1.6. Experimental procedure for the relative expression analysis of microRNA biogenesis and P-body/stress granule-associated proteins (Chapter 2). Figure created using BioRender.com. 

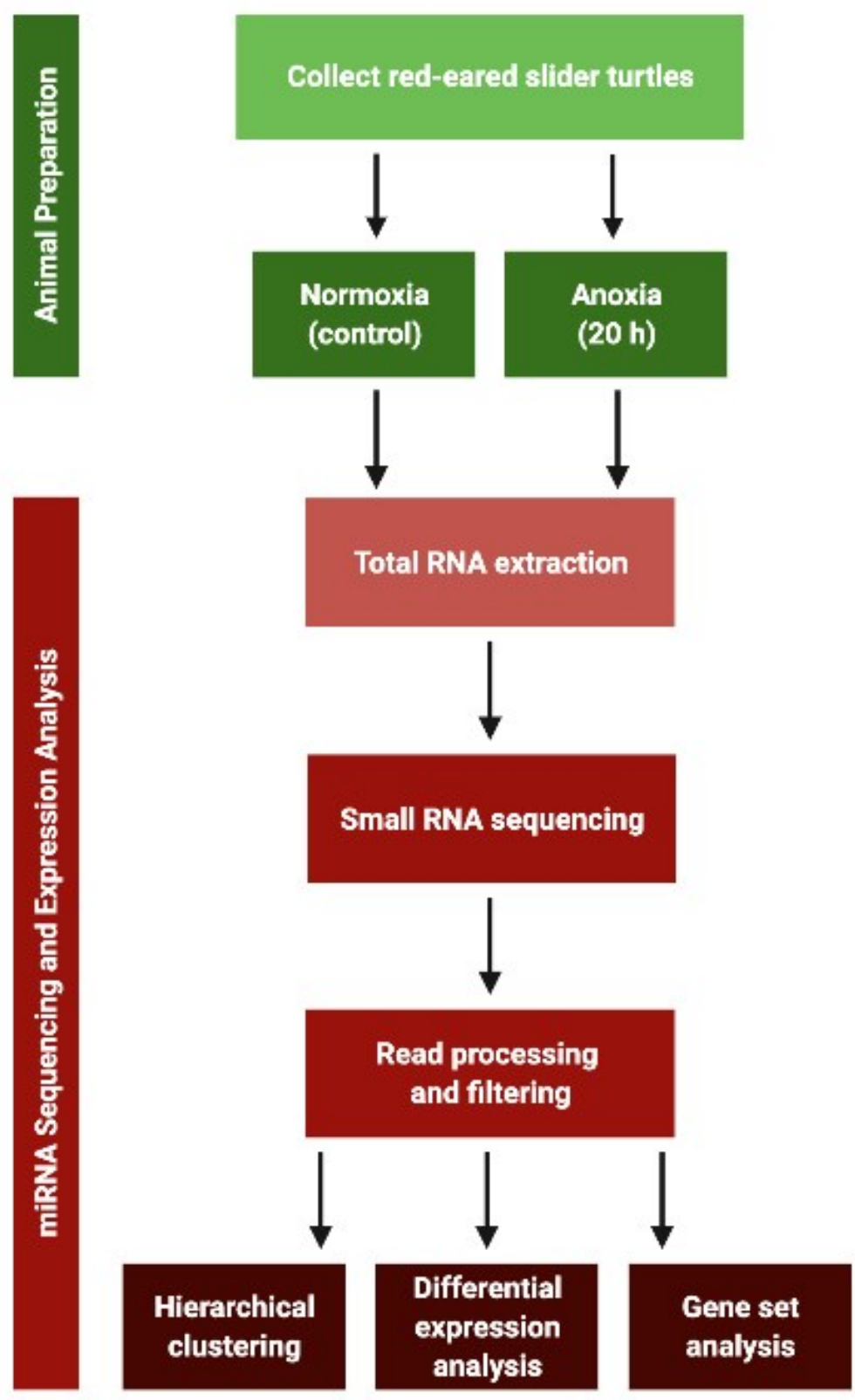

Figure 1.7. Experimental procedure for microRNA sequencing and bioinformatic analyses (Chapter 3). Figure created using BioRender.com. 


\section{CHAPTER 2}

\section{MicroRNA Biogenesis and Messenger RNA Fate}




\subsection{INTRODUCTION}

Red-eared slider turtles (Trachemys scripta elegans) are capable of surviving extended periods of oxygen deprivation without suffering any apparent tissue damage. These turtles experience both short-term hypoxic conditions during breath-hold diving, as well as long-term anoxic conditions over the winter months while they hibernate underwater for weeks to months at a time. During winter hibernation, these turtles can be unable to surface for air if bodies of water are ice-locked and the water can soon become hypoxic/anoxic to an extent that turtles are incapable of extracting sufficient oxygen using extrapulmonary methods (Jackson \& Ultsch, 2010; Storey, 2007). As such, extended underwater submergence results in the turtles' blood oxygen levels eventually dropping to near zero Torr (Jackson, 1968; Ultsch, 1989). In animals that are not anoxia-tolerant, this lack of oxygen would result in grievous tissue damage, likely leading organismal death after even a short period of time. However, the red-eared slider turtles can survive oxygen deprivation for days, weeks or even months, before quickly returning to normal functioning once they have access to ample oxygen again.

This ability is underscored by a set of complex mechanisms that serve to protect the turtle from various cellular insults that can accompany repeated anoxia-reoxygenation cycles. These adaptations mechanisms include the ability to buffer the lactate generated during anaerobic glycolytic fermentation to prevent lethal acidosis, chaperone proteins to protect and refold proteins, and extensive constitutive and inducible antioxidant defences to mitigate macromolecule damage caused by reactive oxygen species (ROS) (Breedon et al., 2021; Davis \& Jackson, 2007; Kesaraju et al., 2009; Krivoruchko \& Storey, 2010d; Willmore \& Storey, 1997a). Besides protective mechanisms such as these, the red-eared 
slider turtle also has the amazing ability to reduce its metabolic rate by approximately $90 \%$ compared to its normoxic rate (Hochachka, 1988; Jackson, 1968; Storey, 2007). This reduces the cellular demand for adenosine triphosphate (ATP) to levels that can be satisfied by anaerobic metabolic pathways, which are fueled by the high organ glycogen reserves, particularly those in the liver (Jackson \& Ultsch, 2010; Storey, 2004). Metabolic rate depression (MRD) is crucial to the survival of extended bouts of anoxia because if the balance of cellular energetics (i.e. the rate of ATP production versus consumption) is disrupted, the overconsumption of ATP will rapidly lead to glycogen depletion and organismal death. A major characteristic of MRD is the global suppression of nonessential, energetically expensive processes such as protein translation and turnover, and cell cycle progression (Storey \& Storey, 2004). However, despite needing to conserve as much energy as possible via the global suppression of gene expression, there are still a number of important pro-survival pathways that must be maintained or upregulated in order to ensure that the animal can survive prolonged stress exposure (Storey \& Storey, 2004). Thus, in addition to global MRD, there must also be a simultaneous reprioritization of energy usage towards the selective expression of certain stress-responsive genes.

MRD and metabolic reorganization is accomplished through the coordinated use of various regulatory mechanisms (Storey \& Storey, 2004). In particular, post-transcriptional inhibition of gene expression via microRNA (miRNA) is of particular interest due to their ability to rapidly and reversibly alter gene expression in response to external stimuli (O'Brien et al., 2018). miRNAs are a class of short ( $22 \mathrm{nt})$ non-coding RNA that are highly conserved between species and that function to repress the translation of mRNA into protein through miRNA:mRNA binding (Bartel, 2004; O'Brien et al., 2018). miRNA 
regulation has been implicated in MRD of numerous stress-tolerant organisms including wood frogs, milk snails, mouse lemurs, brown bears, and naked mole rats (Hadj-Moussa et al., 2020, 2021; Hoyeck et al., 2019; Luu et al., 2020). Notably, previous studies have shown that miRNAs are important regulators of cell proliferation and metabolic processes in red-eared sliders in response to anoxia (Biggar \& Storey, 2012, 2017; Zhang et al., 2013). Given their importance to $\mathrm{MRD}$, the regulation of miRNA production is a critical step for analysis to determine how these turtles alter miRNA expression under anoxia.

miRNAs are produced endogenously primarily through the canonical biogenesis pathway (Figure 1.3). Firstly, nuclear RNA polymerase II transcribes a miRNA-coding gene into the primary miRNA (pri-miRNA) transcript, which is then cleaved by the microprocessor complex (Ha \& Kim, 2014). This complex consists of Drosha-a ribonuclease (RNase) III enzyme — and DiGeorge syndrome critical region 8 (DGCR8)— a non-catalytic subunit containing two double-stranded RNA (dsRNA)-binding domains (Ha \& Kim, 2014; O'Brien et al., 2018). Drosha crops the 5' and 3' single-stranded trailing ends of the pri-miRNA, leaving only the $\sim 70 \mathrm{nt}$ stem-loop structure of the precursor miRNA (pre-miRNA) (Finnegan \& Pasquinelli, 2013; O’Brien et al., 2018). Following cropping, the pre-miRNA is exported through a nuclear pore into the cytoplasm via Exportin-5 (XPO5) in a Ran-GTP-dependent manner (Finnegan \& Pasquinelli, 2013; O'Brien et al., 2018). Once in the cytoplasm, the terminal loop of the pre-miRNA is cleaved by RNase III Dicer to form a $\sim 22$ nt dsRNA duplex of mature miRNA (Finnegan \& Pasquinelli, 2013; O'Brien et al., 2018). Dicer is aided in this process by its cofactors, TAR RNA-binding protein (TRBP) and protein activator of PKR (PACT), which stabilize Dicer to increase its efficiency (Finnegan \& Pasquinelli, 2013; O'Brien et al., 2018). Following 
cleavage, the miRNA duplex is brought to the RNA induced silencing complex (RISC)loading complex (RLC), which contains Dicer, TRBP, and Argonaute (AGO) proteins, and one strand (the guide strand) is loaded into AGO to form miRISC (Finnegan \& Pasquinelli, 2013; Koscianska et al., 2011). The remaining strand of the miRNA duplex (the passenger strand/miRNA*) is then degraded (Finnegan \& Pasquinelli, 2013). The guide strand is used by RISC to target messenger RNA (mRNA) that is complementary to the miRNA in order to repress its translation (Finnegan \& Pasquinelli, 2013; O’Brien et al., 2018).

miRNA:mRNA binding suppresses gene expression through mRNA degradation or translational inhibition, depending on the specificity of the miRNA seed sequence (Bartel, 2004; Finnegan \& Pasquinelli, 2013). Perfect binding complementarity leads to AGOmediated mRNA cleavage if the AGO protein in RISC contains catalytic activity (AGO2); otherwise, the mRNA is targeted for deadenylation and degradation (Finnegan \& Pasquinelli, 2013). If the binding is imperfect however, the mRNA is silenced and sent to processing bodies (P-bodies) or stress granules (Figure 1.5) (Bartel, 2004; Finnegan \& Pasquinelli, 2013; Leung \& Sharp, 2006). These messenger ribonucleoprotein (mRNP) granules are non-membranous subcellular compartments that act as sites for mRNA processing (Anderson \& Kedersha, 2008). The main function of these foci is temporary storage of translationally repressed mRNA; however, P-bodies also serve as sites of mRNA degradation (Kedersha et al., 2005; Standart \& Weil, 2018). Suppressed mRNAs in both types of mRNP granules can undergo translation once normal environmental/cellular conditions are restored (Standart \& Weil, 2018).

P-bodies are highly conserved and constitutively expressed, whereas stress granules form in response to environmental stress (Kedersha et al., 2005; Ross Buchan, 2014; 
Standart \& Weil, 2018). The size, number, and protein composition of these granules depends on the particular stress (Buchan \& Parker, 2009; Kedersha et al., 2005; Parker, 2012; Parker \& Sheth, 2007). Sequestering mRNA in mRNP granules after miRNA binding inhibits their translation and effectively conserves the energy that an organism has available during the stress period (Kedersha \& Anderson, 2007; Spriggs et al., 2010; Standart \& Weil, 2018). With their connection to miRNA gene silencing, it is not surprising that RISC proteins have been shown to localize to both P-bodies and stress granules under some stress conditions (Gilks et al., 2004; Kedersha et al., 1999; Leung \& Sharp, 2006; Tourrière et al., 2003). GW182-also known as trinucleotide repeat-containing gene 6A protein (TNRCG6A) - is required for miRNA-mediated translational repression of mRNAs (Jakymiw et al., 2005). GW182 associates with mRNA-bound AGO proteins to recruit poly(A)-deadenylase complexes and act as a scaffold for P-body formation, with studies showing that it aids in efficient miRNA gene silencing (Eystathioy et al., 2003; Jakymiw et al., 2005; O'Brien et al., 2018). Additionally, the 5'-3' Exoribonuclease 1 (XRN1) protein is crucial to the P-body's decay function as it is responsible for mRNA degradation (Jain \& Parker, 2013; Kedersha \& Anderson, 2007). The RISC associated protein, poly(A) binding protein cytoplasmic 1 (PABPC1), improves the efficiency of mRNA deadenylation (Christie et al., 2013; Jonas \& Izaurralde, 2015; O'Brien et al., 2018). Although its deadenylation function is important for XRN1-mediated mRNA decay, PABPC1 predominantly localizes in stress granules, along with other proteins such as Tcell-restricted intracellular antigen (TIA)-related protein (TIAR), and TIA-1 (Buchan \& Parker, 2009; Hoyle et al., 2007; Kedersha et al., 2005; Kedersha \& Anderson, 2007). PABPC1 promotes stress granule assembly when it interacts with mRNP oligomers that 
form as the aggregation prone TIAR/TIA-1 proteins join the stalled 48S pre-initiation complex which remains bound to the 5' UTR of the mRNA (Anderson \& Kedersha, 2008; Dang et al., 2006; Mazroui et al., 2006). The mRNA transcripts contained within these granules are stored until conditions are favourable for translation to resume, or they can be transferred to P-bodies to undergo degradation (Anderson \& Kedersha, 2008; Kedersha \& Anderson, 2007; Mollet et al., 2008; Standart \& Weil, 2018).

Altogether, strictly controlled biogenesis and maturation of miRNA is crucial to the normal functioning of a cell, and to an organism's ability to rapidly alter gene expression in response to environmental factors. Changing the expression of key biogenesis proteins can lead to alterations in miRNA expression, and thus, expression of target mRNAs. Due to this, it is critical to understand how stress exposure alters the regulation of miRNA synthesis through the differential expression of key proteins associated with miRNA biogenesis. Regulation of miRNA synthesis via the differential expression of key biogenesis proteins has previously been analyzed in the brain of wood frogs (Rana sylvatica) as a response to freezing stress (Hadj-Moussa \& Storey, 2018), but this process has not previously been assessed in an anoxia tolerance species such as the red-eared slider turtle. Hence, the primary aim of this study was to determine how the relative expression of proteins associated with miRNA biogenesis change in response to anoxia and reoxygenation in turtle liver and red muscle. Additionally, a preliminary analysis of mRNA fate following miRNA targeting was performed by assessing the expression of a select few P-body and stress granule proteins to infer whether these sites of mRNA decay/translational inhibition are altered during anoxia. As outlined in the hypothesis in 
Section 1.6.1, it was expected that miRNA biogenesis and mRNP granules would show tissue-specific expression profiles in response to anoxia and reoxygenation.

\subsection{MATERIALS AND METHODS}

\subsubsection{Animal experiments}

All turtle experimentation was conducted following the protocol outlined in a previous study (Wijenayake et al., 2018). Briefly, adult red-eared slider turtles (Trachemys scripta elegans) weighing $850 \pm 131 \mathrm{~g}$ with mean plastron lengths of $16.5 \pm 0.81 \mathrm{~cm}$ were used in this study. Turtles were maintained in large tanks of dechloraminated water at $5{ }^{\circ} \mathrm{C}$ for approximately two weeks prior to experimentation. Turtles were fed Wardley Reptile Ten Floating Food Stick (New Jersey, USA) ad libitum during this period. Subsequently, turtles were moved into smaller containers in $5{ }^{\circ} \mathrm{C}$ incubators and held for two days. Normoxic turtles were sampled from this group.

Turtles in the experimental anoxia group were treated by submergence in sealed containers (two turtles per container) of $40 \mathrm{~L}$ of deoxygenated water (previously bubbled for $\sim 6 \mathrm{~h}$ with $100 \%$ nitrogen gas). Wire mesh was placed approximately $10 \mathrm{~cm}$ below the water line to prevent turtles from surfacing during the anoxic exposure. Tanks were maintained at $5{ }^{\circ} \mathrm{C}$ and nitrogen gas bubbling was continued for the first $2 \mathrm{~h}$. Turtles were samples after $20 \mathrm{~h}$ of anoxic submergence.

The aerobic recovery turtle group were treated to anoxia exposure as described above before being transferred to containers (two turtles per container) of normal water in $5{ }^{\circ} \mathrm{C}$ incubators with full access to breathing air. Turtles were allowed to recover from the anoxic episode for $5 \mathrm{~h}$ before being sampled. 
All animals were euthanized by decapitation and then tissues were rapidly dissected, and flash frozen in liquid nitrogen. Tissue samples were stores at $-80{ }^{\circ} \mathrm{C}$ until use. Experiments had the prior approval of the Carleton University Animal Care Committee (protocol \#106937) and followed guidelines set by the Canadian Council on Animal Care.

\subsubsection{Total protein extraction}

Total soluble protein extracts were prepared from turtle liver and red skeletal muscle of normoxic control, $20 \mathrm{~h}$ anoxia, and $5 \mathrm{~h}$ aerobic recovery conditions. Samples of frozen tissues $(\sim 500 \mathrm{mg})$ were crushed with a mortar and pestle under liquid nitrogen. Samples were then homogenized 1:2 w/v using a Polytron PT10 homogenizer (Kinematica, Werkstrasse, Switzerland) and ice-cold homogenization buffer (20 mM 4-(2hydroxyethyl)-1-piperazineethanesulfonic acid [HEPES], $200 \mathrm{mM} \mathrm{NaCl}, 0.1 \mathrm{mM}$ ethylenediaminetetraacetic acid [EDTA], $10 \mathrm{mM} \mathrm{NaF}, 1 \mathrm{mM} \mathrm{Na} \mathrm{VO}_{4}, 10 \mathrm{mM} \beta$ glycerophosphate, $\mathrm{pH}$ 7.4) with a few crystals of phenylmethylsulfonyl fluoride (PMSF) and $1 \mu \mathrm{L} / \mathrm{mL}$ of protease inhibitor (BioShop Canada Inc., Burlington, ON; Cat\# PIC002) added immediately before homogenization. Homogenates were centrifuged at 11,000 x g for $15 \min \left(4{ }^{\circ} \mathrm{C}\right)$ in a Biofuge 15 (Baxter CanLab, Brampton, ON), and the supernatants containing soluble proteins were collected in a new tube.

Protein concentrations for each sample were quantified via the BioRad protein assay (BioRad Laboratories, Hercules, CA; Cat\# 5000002) as per the manufacturer's instructions. Absorbance was measured at $595 \mathrm{~nm}$ using a BioTek (Winooski, VT) PowerWave HT microplate spectrophotometer. Concentrations were adjusted to $10 \mu \mathrm{g} / \mu \mathrm{L}$ using homogenization buffer, then aliquots were mixed $1: 1 \mathrm{v} / \mathrm{v}$ with sodium dodecyl sulfate 
(SDS) buffer (100 mM Tris-HCl, 4\% w/v SDS, 20\% v/v glycerol, 0.2\% w/v bromophenol blue, $10 \% \mathrm{v} / \mathrm{v} \beta$-mercaptoethanol, $\mathrm{pH} 6.8$ ) to give a final sample concentration of $5 \mu \mathrm{g} / \mu \mathrm{L}$. Samples were then boiled for $10 \mathrm{~min}$ to denature and linearize the proteins, before being stored at $-40{ }^{\circ} \mathrm{C}$ until use.

\subsubsection{Immunoblotting}

Western blotting was used to examine relative levels of various proteins in liver and red muscle from normoxic control, $20 \mathrm{~h}$ anoxia, and $5 \mathrm{~h}$ aerobic recovery red-eared slider turtles. Equal amounts $(25 \mu \mathrm{g})$ of protein homogenates were loaded onto $6-15 \%$ (depending on the target protein) discontinuous SDS polyacrylamide gels with a $5 \%$ upper stacking gel. Upper stacking gels consisted of 5\% acrylamide v/v, $1 \mathrm{M}$ Tris buffer (pH 6.8), $0.1 \%$ SDS, $\quad 0.1 \%$ ammonium persulphate (APS), and $0.1 \% \quad \mathrm{~N}, \mathrm{~N}, \mathrm{~N}^{\prime}, \mathrm{N}^{\prime}-$ tetramethylethylenediamine (TEMED), while the lower resolving gels consisted of 6-15\% acrylamide $\mathrm{v} / \mathrm{v}, 1.5 \mathrm{M}$ Tris buffer (pH 8.8), 0.1\% SDS, 0.1\% APS, and 0.1\% TEMED. A $5 \mu \mathrm{L}$ aliquot of either PiNK Plus pre-stained protein ladder (10.5-175 kDa; FroggaBio, Toronto, ON; Cat\# PM005-0500) or BLUeye pre-stained protein ladder (11-245 kDa; FroggaBio; Cat\# PM007-0500) was loaded in one lane, depending on the size of the protein of interest. After sample loading, gels were run in 1x running buffer $(25 \mathrm{mM}$ Tris-base [pH 6.8], $190 \mathrm{mM}$ glycine, $0.1 \% \mathrm{w} / \mathrm{v}$ SDS) at $180 \mathrm{~V}$ for $1-4 \mathrm{~h}$ at $4{ }^{\circ} \mathrm{C}$ using a BioRad MiniPROTEAN 3 System.

After electrophoresis, resolved gels were transferred to $0.45 \mu \mathrm{m}$ pore polyvinylidene difluoride (PVDF) membranes (Immobilon-P Transfer Membrane; MilliporeSigma, Darmstadt, Germany; Cat\# IPVH85R) in prechilled transfer buffer (25 $\mathrm{mM}$ Tris-base [pH 8.8], $192 \mathrm{mM}$ glycine $10 \% \mathrm{v} / \mathrm{v}$ methanol) at $60-160 \mathrm{~mA}$ for $1.5-16 \mathrm{~h}$ 
$\left(4^{\circ} \mathrm{C}\right)$. PVDF membranes were then washed in Tris-buffered saline and Tween-20 (TBST) (20 mM Tris-base [pH 7.6], $140 \mathrm{mM} \mathrm{NaCl,} \mathrm{0.05 \%} \mathrm{v/v} \mathrm{Tween-20,} \mathrm{90 \%} \mathrm{v/v} \mathrm{ddH} 2 \mathrm{O}$ ) for 5 min before being incubated in non-fat milk $(1-10 \% \mathrm{w} / \mathrm{v}$ in TBST) for $30 \mathrm{~min}$ on a rocker at room temperature (RT). Milk blocking was performed to limit non-specific binding of primary and secondary antibodies. Membranes were washed in TBST 3 x 5 min, and then incubated with $5 \mu \mathrm{L}$ the primary antibody of interest $(1: 1000 \mathrm{v} / \mathrm{v}$ dilution in TBST) on a rocker overnight at $4{ }^{\circ} \mathrm{C}$. Complete primary antibody supplier information can be found in Appendix B. Antibody specificity was determined prior to immunoblotting by aligning the antibody immunogen sequence to the red-eared turtle genome using the National Center for Biotechnology Information (NCBI) basic local alignment search tool (BLAST) (https://blast.ncbi.nlm.nih.gov/Blast.cgi) as outlined in Appendix C. If no epitope was provided on the supplier website, multispecies alignments were used, and only proteins with high sequence conservation were used as targets for this study.

After primary antibody probing, membranes were washed in TBST 3 x 5 min, and then incubated at RT for 30 min with horseradish peroxidase (HRP)-conjugated secondary antibody (1:8000 v/v in TBST; BioShop) with the secondary antibody depending on the primary antibody used. A complete list of optimized immunoblotting conditions and secondary antibodies can be found in Appendix D. Membranes were washed in TBST $3 \mathrm{x}$ 5 min again prior to being visualized via the ChemiGenius BioImaging System (Syngene, Frederick, MD). After visualization, membranes were stained with Coomassie blue dye $(0.25 \% \mathrm{w} / \mathrm{v}$ Coomassie brilliant blue, $7.5 \% \mathrm{v} / \mathrm{v}$ acetic acid, and 50\% v/v methanol) for 30

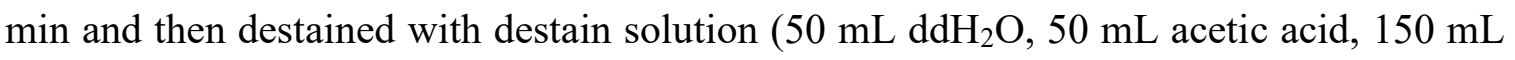


methanol) for 10 min to visualize total protein levels, which was used to standardize the protein bands of interest.

\subsubsection{Data quantification and statistics}

For quantification of relative protein levels, intensities of immunoreactive protein bands of interest were quantified with enhanced chemiluminescence (ECL) using $\mathrm{H}_{2} \mathrm{O}_{2}$ and luminol via the ChemiGenious BioImaging System and GeneTools Software (Syngene). Standardization of protein sample loading was performed using a group of several Coomassie blue stained bands in each lane whose intensity were unchanged between experimental treatments and across the multiple samples. Standardized band intensities were calculated as the ratio of immunoreactive band intensity versus Coomassie blue band intensity. Data were statistically analyzed using one-way analysis of variance (ANOVA) with Dunnett's post hoc test to compare experimental and control values, and results were considered significantly different when $p<0.05$. Statistical analyses were performed using the RBioplot statistical and graphing software package (Zhang \& Storey, 2016). Immunoblot data is expressed as mean values $\pm \operatorname{SEM}(n=4)$, relative to control values.

\subsection{RESULTS:}

\subsubsection{Liver microRNA biogenesis protein expression}

The relative abundance of key proteins involved in the miRNA biogenesis and maturation pathway were measured in liver of normoxic control, $20 \mathrm{~h}$ anoxic, and $5 \mathrm{~h}$ reoxygenated red-eared slider turtles using immunoblotting (Figure 2.1). Levels of the microprocessor complex proteins, Drosha and DGCR8, decreased significantly during anoxia relative to control values, decreasing to $0.29 \pm 0.04$ and $0.57 \pm 0.03$ respectively. 
These decreased levels were sustained into aerobic recovery, with Drosha at $0.33 \pm 0.06$ and DGCR8 at $0.28 \pm 0.03$, as compared with controls. XPO5 protein levels remained unchanged over anoxia and aerobic recovery, whereas Ran-GTP levels decreased to 0.60 \pm 0.10 of control values during anoxia, and to $0.39 \pm 0.09$ during reoxygenation. Relative protein levels of Dicer were unchanged as compared with control values during anoxia and aerobic recovery. However, TRBP and PACT protein levels increased significantly to 3.35 \pm 0.41 and $1.83 \pm 0.19$ over controls during anoxia, respectively, before returning to control values during recovery. Relative protein levels of AGO1 and AGO2, as well as their relative phosphorylation levels, $\mathrm{p}-\mathrm{AGO} 2^{\mathrm{Ser} 387}$, and $\mathrm{p}-\mathrm{AGO} 2^{\mathrm{Tyr} 393}$, remained constant during anoxia and reoxygenation. Levels of AGO3 and AGO4 proteins significantly decreased during anoxia to $0.66 \pm 0.05$ and $0.17 \pm 0.03$ of control values, respectively, but whereas AGO3 levels rose again to control values during recovery, AGO4 remained low at $0.25 \pm 0.02$ relative to the control.

\subsubsection{Muscle microRNA biogenesis protein expression}

Immunoblotting analysis of miRNA biogenesis and maturation proteins was also performed for red muscle of normoxic control, anoxic, and reoxygenated turtles (Figure 2.2). Levels of the microprocessor proteins, Drosha and DGCR8, were unchanged form control levels during anoxia, but significantly increased to $1.77 \pm 0.13$ and $1.64 \pm 0.07$-fold over controls, respectively, during aerobic recovery. Relative protein levels of XPO5 did not change during anoxia or recovery. Ran-GTP protein levels increased to $1.28 \pm 0.03$ during anoxia before dropping to $0.76 \pm 0.02$ of control values during reoxygenation. During anoxia, Dicer relative protein levels rose significantly to $2.11 \pm 0.12$ of control and remained elevated at $2.45 \pm 0.10$ into aerobic recovery. Levels of TRBP and PACT did not 
change during anoxia, but both increased significantly in recovery to $1.37 \pm 0.08$ and 1.26 \pm 0.08 of controls, respectively. Protein levels of AGO1, AGO2, AGO3, and AGO4, as well as relative phosphorylation levels of $\mathrm{p}-\mathrm{AGO} 2^{\mathrm{Ser} 387}$ and $\mathrm{p}-\mathrm{AGO} 2^{\text {Tyr393 }}$, remained unchanged during anoxia and aerobic recovery.

\subsubsection{Liver P-body and stress granule protein expression}

The relative abundance of select P-body and stress granule proteins in normoxic, anoxic, and reoxygenated turtle liver is shown in Figure 2.3. The P-body proteins GW182 and XRN1 were both unchanged from control levels during anoxia but increased by 1.69 \pm 0.06 and $2.36 \pm 0.20$-fold, respectively, over controls in liver from the aerobic recovery group. The stress granule proteins PABPC1, TIAR, and TIA-1 all decreased under anoxia to $0.79 \pm 0.07,0.65 \pm 0.05$, and $0.60 \pm 0.09$, respectively and remained low after $5 \mathrm{~h}$ of aerobic recovery at $0.58 \pm 0.06,0.48 \pm 0.03$, and $0.54 \pm 0.02$, respectively, as compared to the normoxic controls.

\subsubsection{Muscle P-body and stress granule protein expression}

Immunoblot analysis of red muscle P-body and stress granule proteins from normoxic, anoxic, and reoxygenated turtles is shown in Figure 2.4. GW182 levels significantly decreased during anoxia $(0.33 \pm 0.05)$ and recovery $(0.41 \pm 0.06)$ as compared to control levels, whereas XRN1 levels remained unchanged under both experimental conditions. Levels of PABPC1 were not significantly altered under anoxia but increased by $1.71 \pm 0.12$-fold over controls during aerobic recovery. TIAR and TIA-1 relative protein levels did not change during either anoxia or reoxygenation. 


\subsection{DISCUSSION}

Due to their critical role in gene silencing and their ability to rapidly and reversibly alter gene expression, miRNAs are an excellent regulatory mechanism that can be utilized as part of the biochemical response to extreme environmental stress. The fine tuning of gene expression that miRNA enable maximizes the energetic savings associated with MRD, while also allowing for the expression of key pro-survival genes that are critical to an organism's ability to survive prolonged stress periods without suffering deleterious physiological consequences. This regulation occurs via miRNA:mRNA binding, which silences gene expression by leading to mRNA decay or sequestering for temporary storage. The role of miRNA in anoxia-tolerance has been previously studied in red-eared slider turtles, finding tissue-specific changes in expression and an overall increase in the expression of miRNAs associated with metabolic processes and cell cycle suppression (Biggar \& Storey, 2012, 2017). However, the regulation of miRNA synthesis has yet to be studied in anoxia-tolerant turtles. Given the critical role that miRNA plays in achieving MRD during environmental stress, it was hypothesized that there would be tissue-specific differential regulation of miRNA biogenesis and mRNA fate in response to anoxia and aerobic recovery in the red-eared slider turtle.

\subsubsection{Regulation of microRNA biogenesis}

The rate of miRNA synthesis can be altered by regulating the activity, function, and abundance of proteins associated with various steps of the biogenesis and maturation process (Leung \& Sharp, 2010). This regulation of production rate generally correlates with the cellular abundance of miRNAs, making biogenesis an important aspect of miRNAmediated gene regulation to analyze (Zlotorynski, 2019). In the liver, the data presented 
here showed a contradictory decrease in proteins associated with pri-miRNA processing and nuclear export during anoxia and aerobic recovery, but an increase in proteins associated with pre-miRNA processing during anoxia (Figure 2.1). Reductions in Drosha and DGCR8 contents could lead to a subsequent drop in the cleavage of pri-miRNA into pre-miRNA since these proteins form a complex to remove the trailing ends of the primiRNA duplex (O'Brien et al., 2018). Additionally, the decrease in Ran-GTP expression could lead to diminished rates of pre-miRNA export into the cytoplasm for further processing (Figure 2.1) (O’Brien et al., 2018). Together, these indicate the downregulation of pre-miRNA production and export during anoxia and recovery. Interestingly, this finding contrasted with increases in Dicer cofactors, TRBP and PACT, that stabilize and aid Dicer in its endonucleic excision of pre-miRNA hairpin loops, indicating an increased rate of mature miRNA duplex production (Figure 2.1) (Ha \& Kim, 2014; Koscianska et al., 2011; Ma et al., 2008). This discrepancy could potentially be explained by the fact that miRNA synthesis is a rapid process, and its regulation likely changes over the course of prolonged anoxia exposure. Given that the anoxic time point chosen for this study is late (20 h) within the anoxic episode, there could have been a prior buildup of cytoplasmic premiRNAs that were initially produced at the onset of anoxia and stored for later processing. This pre-production would ensure that there are sufficient stores of pre-miRNA that could be rapidly and energetically inexpensively made into mature miRNA capable of normal mRNA silencing. Indeed, the selective uridylation of the pre-miRNA $3{ }^{\prime}$ tail has been shown to temporarily disrupt miRNA maturation, which could facilitate this pausing effect (Bortolamiol-Becet et al., 2015; Reichholf et al., 2019; Reimão-Pinto et al., 2015). Certainly, it would be interesting to analyze the biogenesis regulation profile and pre- 
miRNA uridylation at several time points during anoxic stress to determine if this is indeed the case.

Following processing by Dicer, the now mature miRNA duplex is unwound, and the guide strand is loaded into AGO to form miRISC (Finnegan \& Pasquinelli, 2013). Relative protein levels of $\mathrm{AGO} 1$ and $\mathrm{AGO} 2$ ( $\mathrm{AGO} 2$ being the only with clear slicer activity) showed no changes in protein expression during anoxia or aerobic recovery (Figure 2.1). This indicates that AGO1/2 miRNA loading, and AGO2-mediated mRNA cleavage may be occurring at the same rate across the anoxia-reoxygenation cycle. Although AGO1 is not as critical as AGO2 to miRNA-mediated mRNA silencing, its constant expression provides additional miRNA loading, allowing for sufficient miRNA:mRNA binding to occur (Wang et al., 2012). The maintenance of AGO2 levels is critical to efficient miRNA biogenesis as AGO2 is the main AGO protein in higher order animals, interacting with approximately $60 \%$ of all miRNAs, as opposed to AGO1/3/4, which interact with less than $\sim 30 \%$ (Wang et al., 2012). This has been demonstrated by studies that have shown that the loss of AGO2 results in reduced miRNA expression (Winter et al., 2009). As such, these AGO2 levels indicate either that there is still significant miRNA production occurring during anoxia/reoxygenation, or that the liver maintains sufficient AGO2 levels to avoid a kinetic bottleneck that would slow miRNA production when the turtle enters the recovery phase after anoxia (Reichholf et al., 2019). Additionally, the activity and function of AGO can be modulated by various modifications; for instance, AGO2 Ser387 phosphorylation results in reduced mRNA endonuclease cleavage, and increased translational repression and localization in P-bodies, whereas AGO2 Tyr393 phosphorylation is associated with decreased binding to Dicer in the RLC 
and, subsequently, decreased pre-miRNA processing (Horman et al., 2013; Shen et al., 2013). Levels of $\mathrm{p}-\mathrm{AGO} 2^{\mathrm{Ser} 387}$ and $\mathrm{p}-\mathrm{AGO} 2^{\mathrm{Ty} r 393}$ remained at normoxic levels during anoxia and aerobic recovery, further indicating that there was no significant change in AGO2 functionality in the liver (Figure 2.1). Interestingly, the relative protein expression of AGO3 and AGO4 both decreased under anoxia, as well during reoxygenation for AGO4 (Figure 2.1). This could likely be explained by the need to conserve as much ATP as possible during the stress event. Due to the fact that $\mathrm{AGO} / 4$ are not necessary for efficient miRNA loading, expression of these AGOs could be downregulated under anoxia as an aid to saving energy for the production of other, more critical, proteins (Wang et al., 2012).

By contrast, significant upregulation was observed in many proteins of the miRNA biogenesis pathway in red muscle during anoxia and/or reoxygenation. Increased levels of both Drosha and DGCR8 during reoxygenation could indicate the upregulation of primiRNA cleavage and pre-miRNA production when oxygen was again available (Figure 2.2) (O'Brien et al., 2018). Although expression of these proteins did not change during anoxia, this could be due to there already being sufficient basal expression to facilitate necessary miRNA synthesis. Next, increased expression of Ran-GTP suggests that nuclear export of pre-miRNAs may be upregulated during anoxia (Figure 2.2). Interestingly, RanGTP levels decreased during aerobic recovery, suggesting decreased export (Figure 2.2); however, this contradictory finding could be explained by a shift to an alternative nuclear export mechanism that is independent of Ran-GTP (Ha \& Kim, 2014). Alternatively, this decrease could signal that nuclear export is a key rate-limiting step that cells exploit to fine tune the rate of miRNA synthesis, in spite of increased expression of multiple other biogenesis proteins. Dicer levels were strongly upregulated in anoxia and aerobic recovery, 
along with TRBP and PACT upregulation during recovery (Figure 2.2). These observations point towards an increase in Dicer-mediated cleavage of pre-miRNA under anoxia, and especially during reoxygenation with enhanced Dicer stability provided by elevated TRBP and PACT, which would serve to further increase the efficiency of this cleavage (Figure 2.2) (Haase et al., 2005; Y. Lee et al., 2006; Winter et al., 2009). None of the AGO proteins, nor of the serine or tyrosine phosphorylated forms of AGO2s were significantly altered in response to anoxia or reoxygenation (Figure 2.2). This could be due to the normoxic AGO expression levels being sufficient to achieve the upregulation of miRNA synthesis, as presumed by the increases in other biogenesis proteins.

Taken together, these results indicate that liver miRNA biogenesis is likely either (1) increased if there are sufficient cytoplasmic pre-miRNA stores to supply Dicermediated cleavage and subsequent mRNA-targeting, or (2) decreased to conserve vital liver function during anoxia/reoxygenation while maintaining expression of critical premiRNA processing proteins to avoid bottlenecks once miRNA synthesis resumes. Analysis of miRNA expression profiles during anoxia and/or aerobic recovery to determine how they differ from normoxia could elucidate which of these theories is most accurate. Although the data suggest an overall reduction in pri-miRNA processing and nuclear export in the liver, future studies should be conducted to interrogate the use of various noncanonical biogenesis pathways that could explain the downregulation of these proteins ( $\mathrm{Ha}$ \& Kim, 2014). In particular, the Drosha/DGCR8-independent biogenesis pathway, and pathways that utilize alternative nuclear export mechanisms should be analyzed to see if they are used in place of or in conjunction with the canonical method (Ha \& Kim, 2014). MiRNA biogenesis appears to be upregulated in red muscle during anoxia and 
reoxygenation as evidenced by the increased expression of multiple proteins at each significant step in miRNA biogenesis. The increased expression of many proteins throughout the biogenesis process in muscle during anoxia/reoxygenation illustrates that miRNA production is enhanced in this tissue. Upregulation of biogenesis indicates that miRNA-mediated post-transcriptional gene silencing serves an important role in the stress response of these turtles, likely aiding in the global suppression of translation in less metabolically active organs. This suppression would lead to great energetic savings which could then be used to fuel key pro-survival processes in tissues more crucial to the turtle's survival (e.g. the liver).

Organ-specific differences in miRNA biogenesis may be attributed to the difference in organ function during anoxic episodes. During periods of oxygen deprivation, turtles depend on carbohydrate fermentation to produce ATP. The liver plays a key role in glycogenolysis and synthesis of glucose molecules that is distributed to all other organs in order to maintain their necessary functions. Thus, it follows that liver is likely not as metabolically suppressed as some other organs. This may explain why miRNA biogenesis was significantly more upregulated in muscle as compared to liver, given that when turtles overwinter at the bottom of ponds or lakes, they are essentially comatose, therefore muscle function would not massively affect their survival whereas liver function certainly would. As such, turtles would need to preserve some level of gene expression in the liver, meaning that miRNAs cannot be as widely used for global silencing as they otherwise would in metabolically arrested organs like muscle. Although little work on environmental stressinduced changes in expression of miRNA biogenesis proteins has been done to date, there is evidence that the brain of frozen wood frogs (Rana sylvatica) is subject to 
downregulation of miRNA biogenesis (Hadj-Moussa \& Storey, 2018). Similar to the findings in turtle liver, reduced biogenesis in frog brain mainly centered on the regulation of proteins associated with pre-miRNA production and nuclear export, indicating that in metabolically and physiologically critical organs such as the liver and brain, this may be an important regulatory target (Hadj-Moussa \& Storey, 2018). Future research on the regulation of biogenesis proteins in other organs and in other stress-tolerant organisms can provide more insight into whether this response is generally conserved, or if there is an organ-, stress-, and animal-specific pattern of biogenesis regulation. Moreover, the expression of a select few miRNA species has been analyzed in anoxic red-eared slider turtles, finding tissue-specific miRNA expression. One study found that both liver and muscle showed upregulation of most of the 11 miRNAs studied, along with the downregulation of more miRNAs in liver than in muscle (Biggar \& Storey, 2017). A further study demonstrated an increase in the expression of one miRNA in both liver and muscle, while another study found that two miRNAs were upregulated in liver but not in muscle in response to anoxia (Biggar \& Storey, 2012; Zhang et al., 2013). Nonetheless, these limited findings do not preclude the idea that a significant number of other miRNA species are upregulated in muscle as indicated by this miRNA biogenesis study, and indeed all of these studies point toward liver miRNA expression also being upregulated in comparison to normoxia. Intriguingly, this seems to support the idea that liver miRNA biogenesis is in fact not significantly decreased during anoxia, but that there are ample stores of cytoplasmic pre-miRNA to continue sufficient mature miRNA expression. Although interesting, these miRNA expression studies are far from comprehensive in terms 
of how many miRNAs were assessed and future studies analyzing complete miRNAomes would provide a clearer picture of how much miRNA biogenesis is actually occurring.

\subsubsection{Messenger RNA fate in P-bodies and stress granules}

In response to environmental or cellular stress, cytoplasmic mRNP granules form as subcellular foci to sequester mRNA (Standart \& Weil, 2018). This can take the form of mRNAs targeted for exonuclease decay in P-bodies, or translationally repressed mRNAs that are temporarily stored in P-bodies or stress granules until favourable conditions are restored (Standart \& Weil, 2018). In the liver, GW182 and XRN1 proteins were similarly expressed, with both increasing during aerobic recovery (Figure 2.3). Both of these proteins are associated with P-body formation and mRNA decay, possibly indicating an increase in the amount of mRNA being targeted to P-bodies for degradation (Standart \& Weil, 2018). GW182 plays a central role in miRNA-mediated gene silencing through its interactions with AGO proteins, and its knockdown is associated with P-body disassembly (J. Liu, Rivas, et al., 2005; Yao et al., 2013). Thus, the overexpression of GW182 highlights the potential increased mRNA binding and P-body formation during reoxygenation. Additionally, the exonuclease XRN1 is responsible for mRNA decay, and its elevated abundance points towards increased degradation (Kedersha \& Anderson, 2007). Increases in both of these proteins strongly suggest that mRNAs targeted by miRNA may be subject to increased P-body localization and exonuclease decay in the liver of red-eared slider turtles during reoxygenation.

This finding contrasts with the coordinated decrease in the expression of the three proteins associated with stress granule formation assessed (PABPC1, TIAR, and TIA-1) in liver during anoxia and recovery (Figure 2.3). PABPC1 complexes with the 3 ' poly(A) 
tails of mRNAs to form mRNP aggregates with TIAR/TIA-1 and leading to the formation of stress granules (Anderson \& Kedersha, 2008; Gilks et al., 2004). Due to its key role in joining the target mRNA to stress granule assembly proteins, the reduction in PABPC1 probably indicates that less stress granule formation is occurring. Stress granule induction also depends on the concentration of the key aggregation proteins, TIAR and TIA-1 (Gilks et al., 2004). Thus, it is likely that the decrease seen in both of these proteins in liver would signify reduced stress granule formation. Although little work has been done on the involvement of P-bodies or stress granules in stress-tolerant organisms, a previous study found no evidence for cytoplasmic stress granule formation in the liver of hibernating thirteen-lined ground squirrels (Ictidomys tridecemlineatus) using indirect fluorescence microscopy and relative protein expression analyses (Tessier et al., 2014). This corroborates the findings regarding decreased stress granule protein expression presented above. However, microscopy experiments are needed to confirm that this is indeed the case in red-eared slider turtles.

Interestingly, the apparent upregulation of P-bodies and downregulation of stress granules in liver is opposite to what was observed in red muscle. Protein levels of muscle GW182 were lower during anoxia and reoxygenation than in normoxia (Figure 2.4), implying that there would be less GW182-AGO2 interactions and thus, less mRNAs localized to P-bodies. Noteworthy however, is that XRN1 did not decrease in expression (Figure 2.4). This suggests that, even though less mRNA is being targeted to XRN1 by virtue of less GW182, that XRN1 was still functioning at basal rates, which may be due to the many roles that XRN1 plays in normal gene expression independent of its actions in sequestering miRNA. Indeed, XRN1 has also been implicated in coordinating 
transcription, mRNA subcellular localization, and translation initiation (Begley et al., 2019; Blasco-Moreno et al., 2019). These results hint that there may be less use of P-bodies in muscle during anoxia, but additional work should be done to elucidate whether a reduction in GW182 expression meaningfully affects mRNA silencing, and if it does in fact represent a transition from the use P-bodies to the use of stress granules as the primary site of mRNA inhibition during anoxia and reoxygenation. Beyond these P-body proteins, PABPC1 expression was found to increase in recovery, whereas TIAR and TIA-1 remained unchanged in anoxia and recovery (Figure 2.4). Upregulated PABPC1 expression would lead to increased mRNA interactions, likely inducing stress granule formation when it interacts with TIAR/TIA-1 (Anderson \& Kedersha, 2008; Gilks et al., 2004). The lack of change in TIAR and TIA-1 expression likely points towards the basal levels of these proteins being adequate for stress granule formation.

The analysis of mRNA fate seems to indicate that red-eared slider turtle liver favours P-bodies during aerobic recovery over stress granules. This is interesting considering the usual shift towards stress granule formation over the use of P-bodies during periods of cellular stress (Kedersha \& Anderson, 2007). Red muscle however, displayed this transition away from P-body prioritization, as expected. These contrary responses are consistent with the notion that mRNP granule induction is indeed tissue-specific, and posits the need for further, more in-depth research into the matter. Overall, this analysis of mRNA fate in liver and muscle of red-eared slider turtles provided an interesting starting point for further research into what becomes of the mRNAs targeted by miRNA silencing. Future studies focusing on the expression of additional P-body/stress granule proteins and/or visualizing the number, size, and localization of these cytoplasmic foci would provide 
additional information about how they are altered during anoxia-reoxygenation cycles and what affect they may have on mRNA fate. These would provide further evidence for altered mRNP granule formation and mRNA sequestration and/or decay in response to anoxic stress in the red-eared slider turtle.

\subsection{CONCLUSIONS}

This study provides the first analysis of the regulation of miRNA biogenesis and the involvement of P-bodies and stress granules in translational inhibition during anoxia stress and aerobic recovery of red-eared slider turtles. Regulated miRNA expression and mRNA suppression is critical to achieving the tissue-specific gene expression that enables turtles to enter MRD and survive prolonged periods of oxygen deprivation. The regulation of miRNA biogenesis in liver showed that the initial steps of miRNA synthesis were downregulated during anoxia and reoxygenation, whereas the later cytoplasmic processing steps were upregulated in anoxia. Taken together, the results indicate that the liver either has decreased miRNA expression in anoxia/reoxygenation as compared to normoxic conditions, or that there was sufficient pre-miRNA production and nuclear export in early anoxia to be able to sustain the upregulation of select miRNAs as seen in previous studies. Additionally, translationally silenced mRNAs in liver appear to be sent to P-bodies over stress granules for storage and/or decay, whose proteins were tandemly downregulated. Conversely, muscle showed clear upregulation of various steps of the biogenesis pathway including pri-miRNA processing, pre-miRNA nuclear export, and pre-miRNA cytoplasmic processing. These upregulated steps suggest increased miRNA synthesis occurring in red muscle during anoxia/reoxygenation. Moreover, muscle seemed to favour increased storage of translationally silenced mRNA in stress granules, while downregulating P-body 
formation. Overall, this study provides new insights into the regulatory mechanisms underlying miRNA synthesis and mRNA storage/degradation in response to anoxia stress and presents interesting areas for future research. 


\subsection{FIGURES}

Liver MicroRNA Biogenesis

A

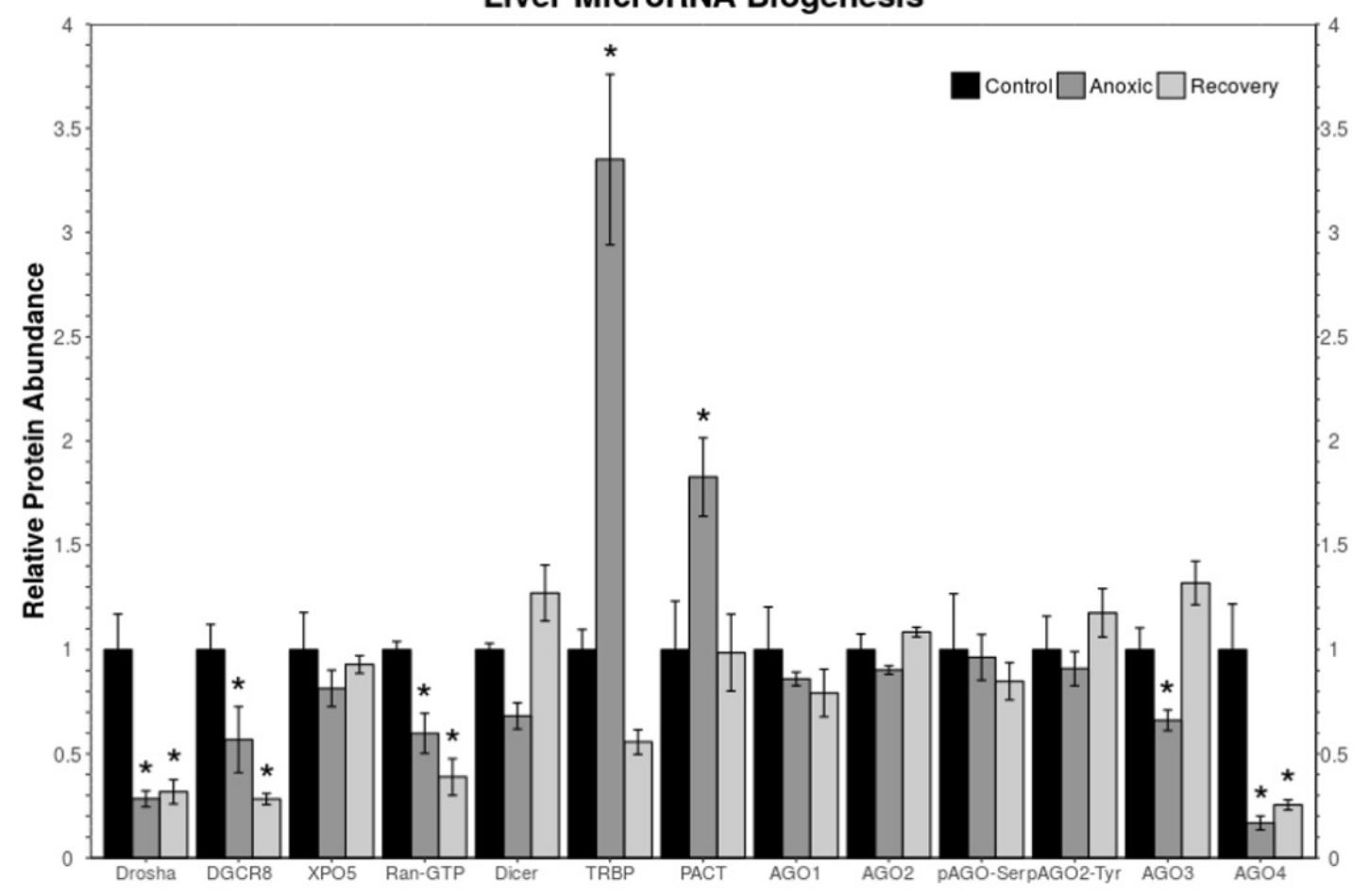

B

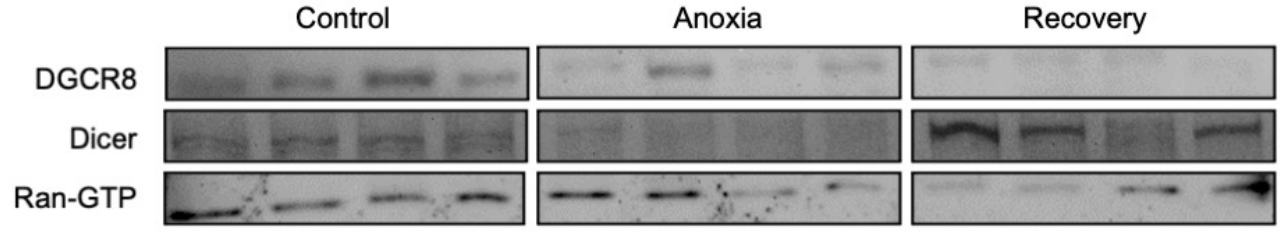

Figure 2.1. Analysis of miRNA biogenesis pathway protein levels in red-eared slider turtle liver under control, anoxia, and recovery conditions. (A) Graph showing relative expression of Drosha, DGCR8, XPO5, Ran-GTP, Dicer, TRBP, PACT, AGO1-4, p$\mathrm{AGO} 2^{\mathrm{Ser} 387}$, and $\mathrm{p}-\mathrm{AGO} 2^{\text {Tyr393 }}$ under normoxic (control), $20 \mathrm{~h}$ anoxic, and $5 \mathrm{~h}$ aerobic recovery conditions. Data are mean $\pm \operatorname{SEM}(n=4$ independent trials). Statistical significance for anoxia or recovery values, relative to the standardized control, was determined using one-way analysis of variance (ANOVA) with Dunnett's post hoc test (* $-p<0.05$ ). (B) Representative immunoblots of selected protein targets are shown for the three experimental conditions. 


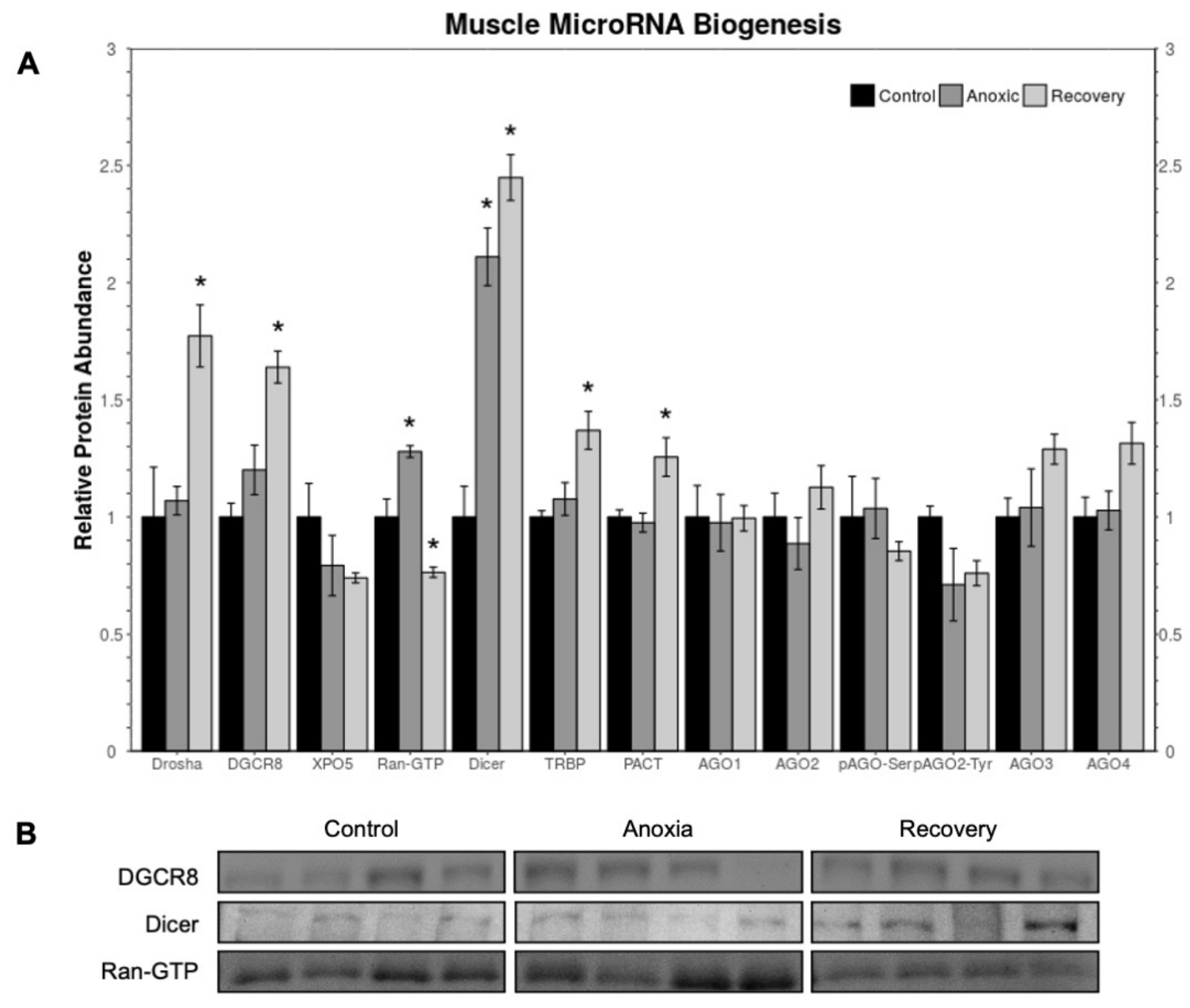

Figure 2.2. Analysis of miRNA biogenesis pathway protein levels in red muscle of redeared slider turtles under control, anoxia, and recovery conditions. Other information as in Figure 2.1. 


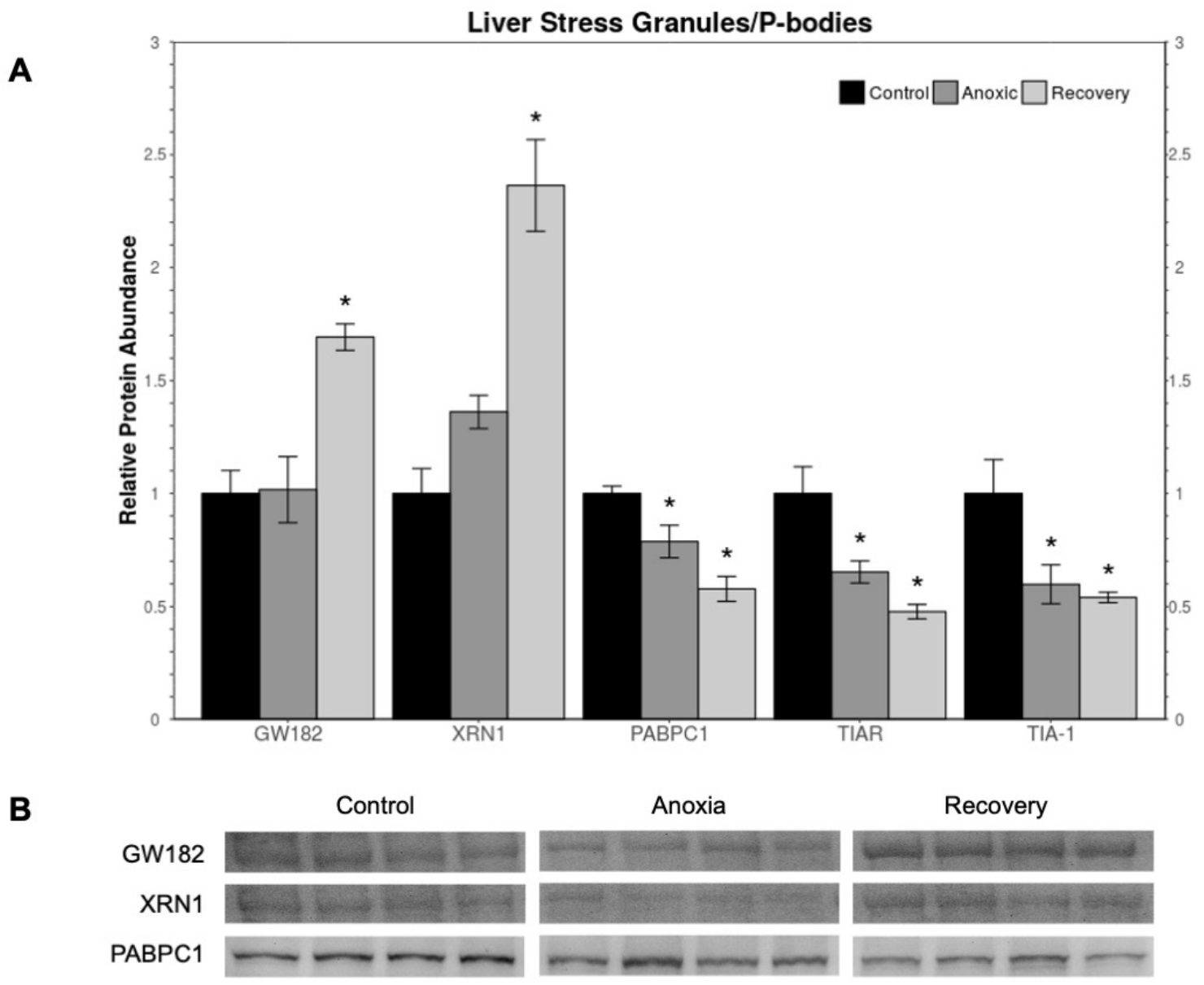

Figure 2.3. Relative expression analysis of proteins associated with P-body and stress granule in red-eared slider turtle liver. Graph shows protein levels of GW182, XRN1, PABPC1, TIAR, and TIA-1 under control, anoxic, and recovery conditions. Other information as in Figure 2.1. 


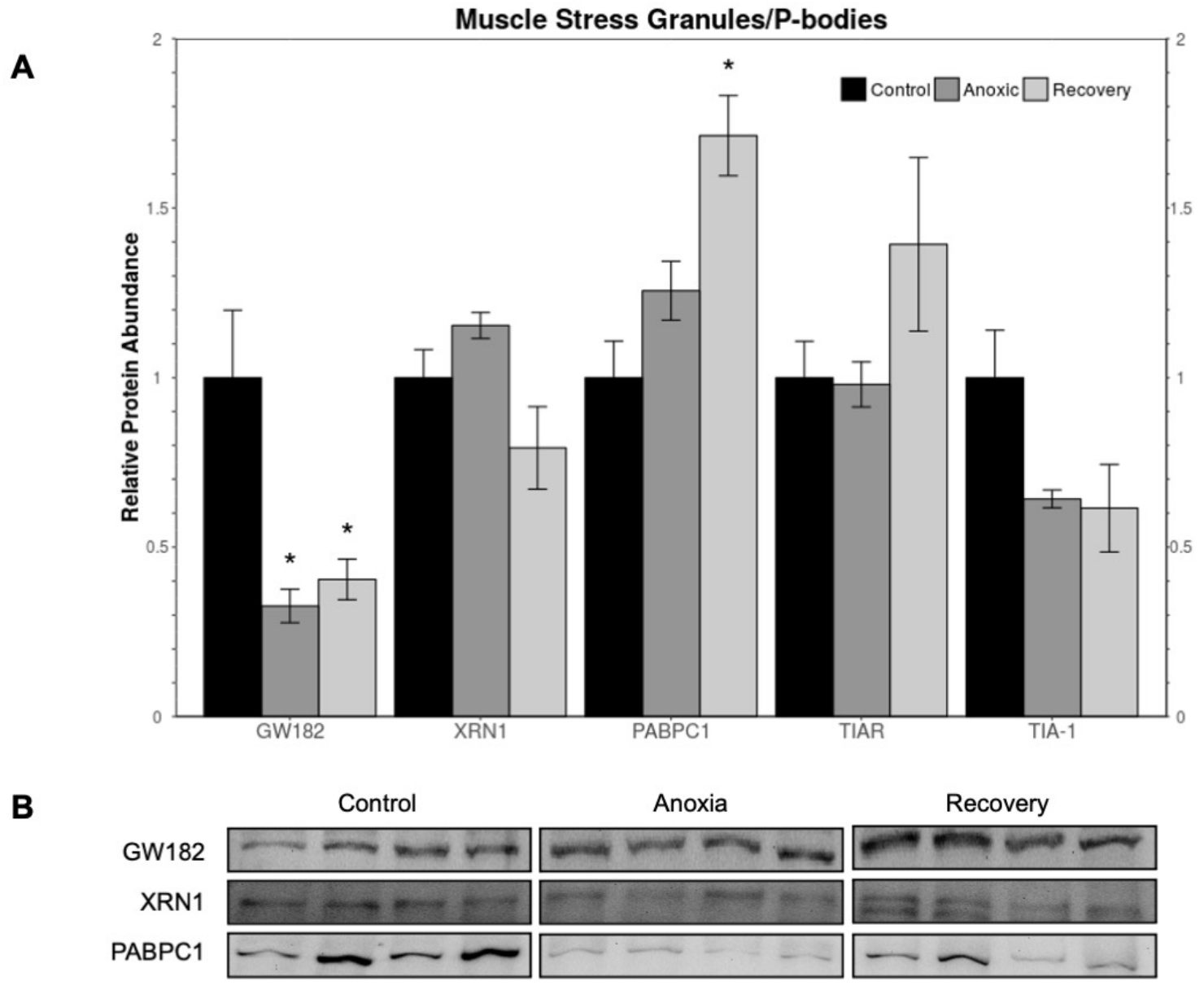

Figure 2.4. Relative expression analysis of proteins associated with P-body and stress granule in red-eared slider turtle red muscle. Other information as in Figure 2.1. 


\section{CHAPTER 3}

\section{MicroRNA Expression in Anoxic Turtle Liver}




\subsection{INTRODUCTION}

Turtles routinely experience short-term hypoxia/anoxia-reoxygenation cycles during breath-hold diving for food or to escape predation. Additionally, certain anoxiatolerant species such as the red-eared slider turtle (Trachemys scripta elegans) are able to survive weeks to months of anoxic conditions during winter hibernation underwater (Jackson, 1968; Ultsch, 1985, 1989). Over the winter, these turtles can become trapped in ice-locked lakes and ponds without access to fresh oxygen leading to blood oxygen levels that eventually drop to near zero (Jackson, 1968; Ultsch, 1989). Amazingly, the turtles are able to survive these prolonged episodes of anoxic submergence without suffering any apparent tissue damage. This ability is in stark contrast to humans and most mammals that, when exposed to even short-term episodes of oxygen-deprivation, typically experience severe organ damage such as ischemia-reperfusion injury (IRI) following heart attack or stroke. Interestingly, the incredible ability to endure prolonged oxygenlimitation/deprivation is found in many species across multiple phyla including red-eared slider turtles, wood frogs, northern crayfish, Humboldt squid, and naked mole rats, and many others (Hadj-Moussa \& Storey, 2020; Storey, 2015). The widespread nature of anoxia tolerance implies the existence of a set of common underlying molecular mechanisms that these organisms are able to manipulate in order to survive extended bouts of oxygen deprivation.

The natural anoxia tolerance of the red-eared slider is facilitated by a number of biochemical adaptations including pro-survival mechanisms such as increased antioxidant defences to prevent damage to macromolecules by reactive oxygen species (ROS), the use of chaperones to stabilize proteins, and lactate buffering to prevent acidosis resulting from 
anaerobic glycolysis (Breedon et al., 2021; Jackson, 2000; Krivoruchko \& Storey, 2010d, 2010c; Willmore \& Storey, 1997b). Additionally, a key mechanism utilized by red-eared sliders during extended anoxia episodes is metabolic rate depression (MRD) (Storey, 2007). Under anoxia, oxidative phosphorylation is halted as there is no oxygen present to complete the final electron transfer in the mitochondrial electron transport chain. Thus, ATP synthesis occurs only through anaerobic reactions, producing lactate, that results in a dramatic decrease in the turtle's capacity for ATP production (Jackson, 2000; Storey, 2007). This is a major issue because if an organism's energetic needs remain the same during bouts of extreme environmental stress whilst their ability to produce ATP sharply decreases - as is the case during anaerobiosis - the animal would not be able to survive the stress for extended periods of time. Thus, MRD is critical for the maintenance of cellular energetics in red-eared slider turtles during long-term anoxic stress, because it enables them to decrease their energy usage in-step with the major drop in ATP production from the less efficient anaerobic fermentation pathway (Storey, 2007). The utilization of MRD as a common stress response is the result of a complex set of regulatory mechanisms. One such mechanism is the microRNA (miRNA)-mediated suppression of gene expression through messenger RNA (mRNA) transcript silencing (Storey, 2015).

First discovered in 1993 in Caenorhabditis elegans, miRNAs are a highly conserved class of short ( 22 nucleotide) non-coding RNA (Bartel, 2004; Finnegan \& Pasquinelli, 2013). miRNAs act as powerful post-transcriptional regulators capable of rapidly suppressing large swathes of the transcriptome in response to environmental stimuli by binding to the 3'-untranslated regions (UTRs) of messenger RNA (mRNA) (Bartel, 2004; Finnegan \& Pasquinelli, 2013). Depending on the degree of complementarity of the 
miRNA seed sequence to its target mRNA, the miRNA:mRNA binding leads to mRNA degradation if perfectly complementary, or if binding is less perfect, to translational repression and mRNA sequestering in stress granules or processing-bodies (P-bodies) (Bartel, 2004; Finnegan \& Pasquinelli, 2013). miRNAs are a crucial regulatory mechanism that stress-tolerant animals utilize in response to severe environmental stress (Biggar \& Storey, 2011, 2018a; Hadj-Moussa \& Storey, 2020). In fact, miRNAs have been shown to be used by a variety of animals to regulate gene expression during stress episodes including: freezing by wood frogs; dehydration of African clawed frogs; hibernation among mouse lemurs, little brown bats, ground squirrels and brown bears; hypoxia/anoxia in crayfish naked mole rats and Humboldt squid (Bansal et al., 2016; Biggar \& Storey, 2014; English et al., 2018; Hadj-Moussa et al., 2018, 2020, 2021; Hawkins \& Storey, 2020; Luu et al., 2020). These animals use miRNA-mediated post-transcriptional regulation to rapidly fine-tune gene expression in response to environmental stress in a tissue-specific manner. This allows them to achieve downregulation of non-critical cellular pathways while reprioritizing pro-survival processes.

Given the widespread use of miRNAs in the stress-responses of various animals, it is evident that miRNAs are a key regulatory mechanism utilized for MRD and metabolic reorganization. Due to their extraordinary ability to endure extended periods of time with oxygen, red-eared slider turtles make an ideal model organism for research into oxygen deprivation and the adaptations that allow these turtles to survive it. Previous studies have characterized the expression of a select few miRNA species in anoxia-exposed red-eared slider turtle livers, with findings that the differential expression of these miRNAs primarily regulates metabolic and cell proliferative processes (Biggar \& Storey, 2012, 2017; Zhang 
et al., 2013). However, these studies only analyzed the expression of specific miRNA species, leaving the rest of the miRNAome unanalyzed to date. With the aim to build our understanding of how these turtles are able to endure long periods of oxygen deprivation, it is necessary to look much more widely at the anoxia-induced miRNA expression profile. A complete picture of which miRNAs are differentially expressed in response to anoxia stress allows us to gain insights into which cellular pathways and processes are important to the anoxia tolerance of red-eared slider turtles and which are targeted for metabolic suppression.

Herein, next-generation sequencing was used to examine the miRNA expression profile in liver of red-eared slider turtles in response to anoxia. Bioinformatic analyses were used to assess the differential expression of miRNAs during anoxia compared to normoxic controls. These results were subsequently used for predictions regarding the cellular functions and pathways that would be most affected by this anoxia-induced miRNA expression profile. Based on previous research, it was expected that energetically expensive pathways such as cell cycle progression and proliferation would be downregulated in anoxia. These findings add to the growing body of knowledge underlying the red-eared slider turtles' ability to endure prolonged anoxic exposure, and of animal adaptations to extreme environmental stresses as a whole.

\subsection{MATERIALS AND METHODS}

\subsubsection{Animal experiments}

All turtle experiments were conducted following previously outlined protocol and had the prior approval of the Carleton University Animal Care Committee (protocol \#106937) following guidelines set by the Canadian Council on Animal Care (Wijenayake 
et al., 2018). Adult red-eared slider turtles (Trachemys scripta elegans) with weights of $850 \pm 131 \mathrm{~g}$ and mean plastron lengths of $16.5 \pm 0.81 \mathrm{~cm}$ were used in this study. All turtles were acclimated in large containers of dechloraminated water at $5{ }^{\circ} \mathrm{C}$ while being fed Wardley Reptile Ten Floating Food Stick (New Jersey, USA) ad libitum for approximately two weeks prior to experimentation. Following acclimatization, turtles were held in smaller holding tanks in $5{ }^{\circ} \mathrm{C}$ incubators for two days, after which the normoxic turtles were sampled from this group. The experimental anoxia group of turtles were treated by submergence in sealed containers (two turtles per container) of $40 \mathrm{~L}$ of deoxygenated water that was previously bubbled with $100 \%$ nitrogen gas for approximately six hours. Wire mesh placed $10 \mathrm{~cm}$ below the water line prevented the turtles from surfacing during the anoxic episode, and tanks were incubated at $5{ }^{\circ} \mathrm{C}$. Nitrogen gas bubbling was continued for the first two hours of anoxic exposure and turtles were sampled after $20 \mathrm{~h}$ of anoxic submergence. Aerobic recovery turtles were treated with the same anoxic exposure but were then transferred to containers (two turtles per container) with normal water held at $5{ }^{\circ} \mathrm{C}$ in incubators. Turtles had full access to air to breathe and were allowed to recover from anoxia for $5 \mathrm{~h}$ before being sampled. All turtles were euthanized by rapid decapitation, then tissues were quickly dissected and flash frozen in liquid nitrogen before being stored at $-80^{\circ} \mathrm{C}$ until use.

\subsubsection{Total RNA isolation}

Total RNA was isolated from liver tissue of normoxic control and $20 \mathrm{~h}$ anoxic redeared slider turtles ( $n=4$ individual animals for each condition) following protocols as described in previous studies (Hadj-Moussa et al., 2020; Hawkins \& Storey, 2020). Approximately $50 \mathrm{mg}$ of frozen tissue was crushed with a mortar and pestle under liquid 
nitrogen and then homogenized using a Polytron PT 1200 homogenizer (Kinematica, Werkstrasse, Switzerland) in $1 \mathrm{~mL}$ of TRIzol Reagent (Invitrogen, Carlsbad, CA; Cat\# 15596-018). Aliquots of chloroform $(200 \mu \mathrm{L})$ were added to each sample prior to centrifugation at $10,000 \mathrm{xg}\left(4^{\circ} \mathrm{C}\right)$ for $15 \mathrm{~min}$. The upper aqueous phase containing total RNA was then transferred to a new microtube and precipitated by adding $500 \mu \mathrm{L}$ of isopropyl alcohol. Samples were incubated at room temperature for 10 min before being centrifuged at $10,000 \mathrm{xg}\left(4^{\circ} \mathrm{C}\right)$ for $15 \mathrm{~min}$. Resulting RNA pellets were washed twice with $70 \%$ ethanol and air-dried for $15 \mathrm{~min}$. RNA pellets were resuspended in $50 \mu \mathrm{L}$ of RNasefree water, and RNA purity and concentration were measured using a Take3 micro-volume quantification plate (BioTek, Winooski, VT; Cat\# 11-120-571) and a PowerWave HT spectrophotometer (BioTek). The 260/280 nm ratios were determined, and samples with ratios of less than 1.8 were excluded from miRNA sequencing analyses. Total RNA integrity was examined via the presence of sharp $28 \mathrm{~S}$ and $18 \mathrm{~S}$ ribosomal RNA (rRNA) bands as seen by electrophoresis on a $1 \%$ agarose gel stained with SYBR Green (Invitrogen; Cat\# S7563). RNA isolates were standardized to a final concentration of 1 $\mu \mathrm{g} / \mu \mathrm{L}$ with RNase-free water, and then frozen and stored at $-80{ }^{\circ} \mathrm{C}$ until use.

\subsubsection{Small RNA sequencing}

Isolated RNA samples were sent to the BC Cancer Genome Sciences Centre (Vancouver, BC) for small RNA-sequencing. Total RNA quality was verified using a Qubit Fluorometer (Invitrogen) and an Agilent 2100 Bioanalyzer with the DNA 1000 chip (Agilent Technologies, Santa Clara, CA; Cat\# 5067-1504), with RIN scores of greater than 7 being the cut-off for library construction. Next, $8 \mu \mathrm{L}$ of total RNA was ligated with 2.5 $\mu \mathrm{M}$ 3' DNA adapter (5'-NNNNNNTGGAATTCTCGGGTGCCAAGTCG-3', 
$\mathrm{N}=$ randomized sequence). Samples were incubated at $70{ }^{\circ} \mathrm{C}$ for $2 \mathrm{~min}$ to denature the RNA secondary structure, and after chilling on ice, $10 \mu \mathrm{L}$ of ligation mix containing truncated T4 RNA Ligase 2 (New England BioLabs, Ipswich, MA; Cat\# M0239S) was added to each reaction. Reactions were incubated at $22{ }^{\circ} \mathrm{C}$ for $1 \mathrm{~h}$ and then purified twice with RNA MagClean DX beads (Aline Biosciences, Woburn, MA; Cat\# C-1005) at room temperature to remove excess adapters. Following purification, 3' adapter-ligated RNAs were ligated to heat-denatured $5^{\prime}$ miRNA adapters (5'UUCCCUACACGACGCUCUUCCGAUCUNNNNNN-3', N = randomized sequence) by T4 RNA ligase (5 U/ $\mu \mathrm{L}$; Invitrogen; Cat\# AM2141) in $20 \mu \mathrm{L}$ reactions, which were then incubated at $37^{\circ} \mathrm{C}$ for $1 \mathrm{~h}$.

During first strand synthesis, the reverse transcriptase (RT) primer (5'CGACTTGGCACCCGAGAATTCCA-3') was incubated with the ligated template strand for 10 min at $65{ }^{\circ} \mathrm{C}$ and then chilled on ice. Maxima H Minus RT $(200 \mathrm{U} / \mu \mathrm{L}$; Thermo Fisher Scientific, Waltham, MA; Cat\# EP0752) was incubated in a $40 \mu \mathrm{L}$ reaction at $44{ }^{\circ} \mathrm{C}$ for $60 \mathrm{~min}$. Complementary DNA (cDNA) was purified with PCRClean DX (Aline Biosciences; Cat\# C-1003) to remove any excess RT primers and reduce off-target product formation. Paired-end primers

$\left(5^{\prime}-\right.$ AATGATACGGCGACCACCGAGATCTACACTCTTTCCCTACACGACGCTCTTCC $\begin{array}{llll}\text { GATCT-3') } & \text { and } & \text { miRNA }\end{array}$ (CAAGCAGAAGACGGCATACGAGATCNNNNNNATCGTACGTCGACTTGGCAC CCGAGAATTCCA) were used in 15 cycles of polymerase chain reaction (PCR). Reactions were performed in $50 \mu \mathrm{L}$ volumes prepared with Phusion Hot Start Flex DNA Polymerase (New England Biosciences; Cat\# M0535S). The amplified library was then 
loaded onto a $12 \%$ polyacrylamide gel electrophoresis (PAGE) gel, and the region containing the miRNA library ( $\sim 150 \mathrm{bp})$ was excised. Ethanol was used to precipitate the size-selected library, which was then purified, and the final small RNA library quality was assessed as before using the Qubit and Agilent assay systems. Sequencing was performed using an Illumina NextSeq500 (San Diego, CA) producing single-end 75 base reads.

\subsubsection{Raw read processing}

Raw read processing was performed as outlined in previous papers using the publicly available RBioMIR pipeline (https://github.com/jzhangc/git_RBioMIR) (HadjMoussa et al., 2020; Zhang et al., 2016). First, adapters were trimmed from all reads using Cutadapt, then Phred quality scores were assessed by FastQC quality reports (http://www.bioinformatics.babraham.ac.uk/projects/fastqc/), and all reads with scores of less than 30 were removed using fastq-mcf (Andrews, 2010; Aronesty, 2011; Martin, 2011). Other non-miRNA small RNA species (piRNA, rRNA, snRNA, snoRNA, and tRNA) were filtered out of the trimmed reads using a negative reference file compiled from the Rfam (https://rfam.xfam.org) and piRNABank (http://pirnabank.ibab.ac.in) databases, and Bowtie (http://bowtie.cbcb.umd.edu) was used to filter and remove any reads that aligned to the negative reference file (Kalvari et al., 2018; Langmead et al., 2009; Sai lakshmi \& Agrawal, 2008). A mature miRNA positive reference file was then constructed using all mature miRNA sequences on miRbase (http://www.mirbase.org/). The filtered reads were aligned against the miRNA positive reference file using Bowtie, with a seed sequence length parameter of 20 nucleotides and only perfect sequence matches being reported. Next, the SAMtools software (http://www.htslib.org) and Unix command line tools were used to sort and determine mature miRNA read counts for the filtered reads $(\mathrm{H}$. 
Li et al., 2009). Read counts for each sample were imported into the $\mathrm{R}$ environment and only miRNAs with more than four reads were used in downstream quantification (R Core Team, 2017; Zhang \& Storey, 2018). All filtered read counts were then normalized via the voom method as previously described (Law et al., 2014; Zhang et al., 2016).

\subsubsection{Differential expression analysis}

Differential expression (DE) analysis of data from normoxic control versus anoxiaexposed turtles was conducted using the linear models for microarray data (limma) R package (https://bioconductor.org/packages/release/bioc/html/limma.html) with a linear model fitting with empirical Bayesian (eBayes) testing (Ritchie et al., 2015). Differentially expressed miRNAs were considered significant if the false discovery rate (FDR)-adjusted $p<0.05$, with a fold change satisfying the following equation:

$$
\left|\log _{2} F C\right| \geq \log _{2} 1.5
$$

\subsubsection{Unsupervised clustering analysis}

The R package gplots (https://github.com/jzhangc/git_RBioArray) was used to perform unsupervised hierarchical clustering using the Ward method on miRNA and animal samples to produce heatmaps with dendrograms (Ward, 1963; Warnes et al., 2015). miRNA expression profiles were further characterized using unsupervised principal component analysis (PCA) on each sample to identify similarities between gene expression patterns and samples (Lenz et al., 2016). PCA analysis reduces the dimensionality of highdimensional datasets by linearly transforming data into uncorrelated latent variables (principal components) that collapse data into a lower dimensional space while preserving the structure and relationships of the original dataset (Lenz et al., 2016). PCA biplots were 
generated by plotting $\mathrm{PC} 1, \mathrm{PC} 2$, and $\mathrm{PC} 3$ - the minimum number of $\mathrm{PC}$ plots representing the most variance — and superimposing PCA score distributions for miRNAs.

\subsubsection{Gene set analysis}

Functional characterization of the anoxic miRNA expression profile was performed using logistic regression-based gene set (GS) analysis via the R package RBiomirGS (https://github.com/jzhangc/git_RBiomirGS) as previously described (Hawkins \& Storey, 2020; Zhang \& Storey, 2018). The RBiomirGS package uses multiple databases to establish miRNA:mRNA interactions; fully conserved human miRNA orthologs were used in this study because of limited $T$. s. elegans annotation and only the top 5\% of predicted miRNA:mRNA interactions were used. After determining miRNA:mRNA interactions, RBiomirGS calculated the miRNA score $\left(S_{m i R N A}\right)$ for each miRNA as previously described (Garcia-Garcia et al., 2016). $S_{m i R N A}$ was calculated using the following equation:

$$
S_{m i R N A}=-\log _{10} p \times \text { signum }\left(\log _{2} F C\right)
$$

where $p$ is the FDR-adjusted $p$-value, and $F C$ is the fold-change of the miRNA between control and anoxic turtles from the differential expression analysis. An mRNA score $\left(S_{m R N A}\right)$ was calculated for each mRNA target using the following equation:

$$
S_{m R N A}=-\sum_{i=1}^{n} S_{m i R N A}^{i}
$$

where $n$ is the number of miRNAs targeting the mRNA, and $S_{m i R N A}{ }^{i}$ is the $S_{m i R N A}$ of the $i^{\text {th }}$ targeting miRNA. The calculated $S_{m R N A}$ scores were then used to determine significantly enriched gene sets (FDR-adjusted $p \geq 0.05$ ) with corresponding model coefficients for each term/pathway using a logistic regression-based method to determine statistical significance 
(Zhang \& Storey, 2018). GS enrichment was performed using Gene Ontology (GO) terms and Kyoto Encyclopedia of Genes and Genomes (KEGG) gene sets (Ashburner et al., 2000; Carbon et al., 2017; Kanehisa \& Goto, 2000). Positive model coefficients (purple) correspond to reduced negative regulation (less inhibition) by miRNA, while negative coefficients (blue) correspond to increased negative regulation (more inhibition) by miRNA during anoxia relative to normoxic control.

\subsection{RESULTS}

\subsubsection{Small RNA sequencing analysis}

Data retrieved from liver of normoxic control and anoxic turtles averaged $25,475,223 \pm 4,000,134$ and $18,673,513 \pm 2,774,381$ raw reads, respectively. Following trimming and quality filtering pre-processing steps (removal of low-quality reads, adapters, sequences under $17 \mathrm{bp}$, and non-miRNA small RNA species), 24,664,880 $\pm 4,033,793$ ( $97 \%$ of total) control sample reads and $17,844,864 \pm 2,677,063$ ( $96 \%$ of total) anoxic sample reads remained. Mapping these reads to the miRbase miRNA database found that $5,643,968 \pm 487,688$ ( $23 \%$ of pre-processed) control sample reads and 4,319,279 $\pm 639,434$ (24\% of pre-processed) anoxic sample reads aligned to 190 known miRNA sequences. However, some lowly expressed miRNAs may not be detected by these analyses due to sequence-dependent adapter ligation bias in small RNA sequencing, resulting in underrepresentation of certain miRNAs (Fuchs et al., 2015; Raabe et al., 2014). Analysis of relative expression was performed between control and anoxic samples instead of comparing expression between miRNA to minimize the effect of this bias. 


\subsubsection{Differential microRNA expression during anoxia}

Of the 190 conserved mature miRNAs identified, 23 ( $\sim 12 \%$ of conserved miRNAs) were significantly differentially expressed in livers from anoxic turtles, as compared to normoxic controls (Figure 3.1). DE analysis indicated that 10 miRNAs were significantly upregulated (purple), and 13 miRNAs were significantly downregulated during anoxia (blue). Upregulated miRNAs included: miR-122b-3p, miR-16-5p, miR-194-5p, miR-7-5p, miR-3529-3p, miR-103b, miR-103a-3p, miR-107, miR-30c-1-3p, and let-7i-5p. Downregulated miRNAs included: miR-214-3p, miR-145-5p, miR-4518, miR-193b-3p, miR-10392-5p, miR-503-5p, miR-3781-3p, miR-1-3p, miR-195-5p, miR-4753-3p, miR206, miR-29a-3p, and miR-33a-5p.

\subsubsection{Hierarchical clustering and principal component analysis}

Unsupervised hierarchical clustering analysis of significantly differentially expressed miRNAs using the Ward method showed that all control samples clustered together, and all anoxic samples clustered together (Figure 3.2a). PCA analyses are used to visually represent relative variability in the sequencing data as linear vectors on a biplot. PCA analysis of red-eared slider turtle liver samples revealed that PC1 explained $46.14 \%$ of the variance in the dataset, while PC2 explained $14.25 \%$ of the variance, for a total of $60.39 \%$ of the variance explained between the two. The PCA biplot also showed that anoxic samples (blue triangles) displayed similar miRNA expression as evidenced by their relatively tight clustering, whereas control samples (red circles) were more variable (Figure 3.2b). 


\subsubsection{Gene set analysis for differentially expressed microRNA}

The gene set enrichment analyses of GO Molecular Function (GO MF), Biological Process (GO BP), and Cellular Component (GO CC), and KEGG pathways are shown in Figures 3.3-3.6. In these plots, positive model coefficients (purple) indicate decreased negative regulation by miRNA (i.e. increased overall expression), whereas negative model coefficients (blue) indicate increased negative regulation by miRNA (i.e. decreased overall expression), relative to the normoxic control group.

GO MF analysis revealed that 58 terms were statistically significantly enriched during anoxia (Figure 3.3). Of these, 52 had negative model coefficients, and six had positive model coefficients. GO BP enrichment showed 273 significantly enriched terms in anoxia as compared to normoxic controls (Figure 3.4). From these, 254 had a negative model coefficient, while 19 had a positive model coefficient. Analysis of GO CC enrichment showed that 71 terms were statistically significantly enriched in response to anoxia (Figure 3.5). Of these terms, 58 had negative model coefficients, while 13 had positive model coefficients. KEGG enrichment analysis found 14 pathways that were statistically significantly enriched during anoxia (Figure 3.6). Of these, 12 had negative model coefficients, and two had positive model coefficients. Overall, GO and KEGG term enrichment was primarily focused on cell cycle processes, protein modifications and turnover, olfactory and stimulus detection, and ribosome regulation. 


\subsection{DISCUSSION}

\subsubsection{MicroRNA expression trends during anoxia}

Global liver miRNAomic profiling identified 190 conserved miRNA from normoxic control and anoxic red-eared slider turtles, with 23 of these miRNAs being differentially expression in response to anoxia (Figure 3.1). Studies have previously analyzed the expression of a total of 14 miRNAs in liver of anoxic red-eared slider turtle: miR-34a (Zhang et al., 2013); miR-16-1 and miR-15a (Biggar \& Storey, 2012); and miR1a, miR-133, miR-17, miR-107, miR-148a, miR-21, miR-103, miR-210, miR-20a, miR365, and miR-29a (Biggar \& Storey, 2017). Most of these miRNAs were found to be differentially expressed and collectively, their expression profiles are tied to the regulation of metabolic and cell proliferation processes (Biggar \& Storey, 2012, 2017; Zhang et al., 2013). Of the miRNAs analyzed through these RT-qPCR in these studies, miR-16-1, miR1a, miR-103, and miR-107, were all also identified via small RNA sequencing herein. The direction of change was consistent between our studies, with miR-1a (miR-1-3p) being downregulated, and miR-16-1 (miR-16-5p), miR-103 (miR-103a-3p), and miR-107 all being upregulated during anoxia.

The conservation of these miRNAs points towards their regulation being an important factor in initiating and maintaining a successful response to anoxia. Differences between the differentially expressed miRNAs identified in this study compared to previous studies may be explained by the fact that a single miRNA can target multiple mRNA species and a single mRNA can be targeted by multiple miRNAs (Bartel, 2004). This multiplicity may result in many miRNAs providing a similar function outcome and thus, the exact miRNA that is expressed is not as critical as the overall downstream effects that 
the miRNAs have on mRNA silencing. As such, it is important to identify which cellular processes are most affected by miRNA regulation. This was done using GS analyses of GO BP, GO MF, GO CC, and KEGG terms (Figures 3.3-3.6), which indicated many enriched functional groups including: (1) cell cycle regulation, (2) protein modification and protein turnover, (3) olfactory receptors and sensory detection, and (4) ribosomal regulation of translation.

\subsubsection{Cell cycle regulation}

Perhaps not surprising, cell cycle progression was clearly altered under anoxia exposure given the numerous associated terms predicted to be downregulated. GO MF indicated that myosin binding, microtubule binding, and cytoskeletal protein binding were downregulated (Figure 3.3). GO BP analysis reported negative model coefficients for cell cycle process, cell division, sister chromatid segregation, mitotic cell cycle checkpoint, regulation of cell cycle, $\mathrm{G}_{1}$ DNA damage checkpoint, mitotic spindle assembly, and microtubule cytoskeleton organization, among others (Figure 3.4). GO CC supports these findings with many cellular compartments including microtubule cytoskeleton, midbody, microtubule, spindle, centrosome, kinetochore, and spindle pole among others having negative coefficients (Figure 3.5). Many additional cell cycle terms were also significantly enriched in the anoxic miRNA profile (Figures 3.3-3.5). Together, these terms comprise many specific functions within the cell cycle including chromosome segregation, cell cycle checkpoints, and cytokinesis, in addition to general terms related to the cell cycle as a whole, indicating overall strong suppression of cell cycling in anoxia. The suppression of cell cycle prevents unwanted cell proliferation in the hypometabolic state, which is supported by the GO BP showing a negative model coefficient for the cell proliferation 
term (Figure 3.4). Additionally, KEGG analyses indicate that multiple terms associated with cancer including prostate cancer, pathways in cancer, and small cell lung cancer were also downregulated (Figure 3.6). One of the key hallmarks of cancer is uncontrolled cell cycling and proliferation, and therefore, miRNA targeting of mRNAs related to cell cycle components is likely associated with these terms, leading to their overall downregulation (Hanahan \& Weinberg, 2011).

The finding that cell cycle processes are a major target of miRNA suppression during anoxia correlates with the requirement for an organism to halt energetically expensive processes to save energy that will be used instead to power pro-survival pathways (Storey, 2007). This downregulation is also supported by previous studies which collectively found evidence for halting of cell cycle progression. A study found that decreased cyclin D1 expression mediates cell cycle arrest in liver and kidney of anoxic redeared slider turtle (Biggar \& Storey, 2012). This study also found that enhanced miR-16 expression may underlie cyclin D1 suppression, a finding that is also supported herein (Figure 3.1) (Biggar \& Storey, 2012). Additionally, increases in multiple repressive cell cycle proteins were found in anoxic turtle liver, potentially indicating $\mathrm{G}_{1}$ arrest (Biggar \& Storey, 2018b). Moreover, research has shown enhanced liver p53 expression during anoxia, that may also correlate to the initiation of cell cycle arrest at the $\mathrm{G}_{1}$ phase (Lakin \& Jackson, 1999; Zhang et al., 2013). Taken together, there is much evidence for cell cycle suppression in liver under anoxic conditions as a mechanism for achieving MRD.

\subsubsection{Protein modification and turnover}

Another molecular process that is suppressed during anoxia via increased miRNA targeting is protein modification. This is exemplified through negative model coefficients 
in the following GO terms: general modification terms (protein modification by small protein conjugation or removal, regulation of transferase activity, and transferase complex; Figures 3.3-3.6), kinase-specific terms (protein serine threonine kinase activator activity, kinase regulator activity, protein serine threonine kinase inhibitor activity, kinase activator activity, and regulation of kinase activity; Figures 3.3-3.6), and ubiquitin-specific terms (ubiquitin like protein transferase activity, ubiquitin like protein conjugating enzyme activity, protein ubiquitination, and ubiquitin ligase complex; Figures 3.3-3.6). There were also additional terms associated with protein modification enriched in all GO analyses, which collectively indicate decreased protein modifications in anoxia. All of these terms relate to the regulation of protein modification, with a particular focus on protein kinases and ubiquitin.

Kinases are a class of enzymes that catalyze protein phosphorylation resulting in either activation or inactivation depending on the phosphorylation site (Berg et al., 2002). Several studies on kinases in the liver of red-eared slider turtles have shown decreased kinase activity after prolonged anoxia exposure, supporting the present sequencing results indicating kinase downregulation (Mattice et al., 2018; Mehrani \& Storey, 1995, 1996). Given that these bioinformatic analyses only provide broad $\mathrm{GO} / \mathrm{KEGG}$ term identification, the exact roles of the affected kinases in controlling protein activity during anoxia remains to be determined as effects are highly dependent on the specific kinase, its target proteins, and whether phosphorylation has a positive or negative effect on protein/enzyme action. However, the apparent overall downregulation of protein kinases is consistent with the need for MRD as a major part of the stress response to anoxia. Ubiquitin is small protein $(8.5 \mathrm{kDa})$ that is used as a "tag" to mark proteins for proteosomal degradation. Ubiquitin 
conjugation to target proteins is achieved via the action of 3 enzymes: ubiquitin-activating enzymes (E1), ubiquitin-conjugating enzymes (E2), and ubiquitin ligases (E3) (Hegde, 2010). Given its function in proteolysis and the significant predicted downregulation of many ubiquitin terms, seen in the present work, it can be proposed that ubiquitin-based proteolysis is inhibited under anoxic conditions and that this is facilitated, at least in part, by the altered hepatic miRNA profile under anoxia in turtle liver that could inhibit ubiquitin production.

The GO CC terms Golgi apparatus, Golgi membrane, and intracellular vesicle also had negative model coefficients indicating suppression by miRNA activity (Figure 3.5). The Golgi apparatus is an organelle responsible for modifying and packaging proteins into vesicles to prepare them for intracellular transport and secretion (Connerly, 2010). With reductions in overall protein modifications, it makes sense that Golgi and vesicles are also suppressed in anoxia. Additionally, lysosomes are targeted by vesicles as locations for macromolecule degradation and the KEGG lysosome pathway was downregulated in anoxia (Figure 3.6) further indicating decreased protein degradation. Taken together, the downregulation of these protein modifications, ubiquitin-mediated proteolysis, and lysosomal degradation terms points towards decreased protein turnover during anoxia. Interestingly, while these results indicate decreased protein turnover, the GO MF term unfolded protein binding is predicted to be downregulated during anoxia (Figure 3.3), which is intriguing because this term is related to the binding of chaperone proteins to stabilize and/or refold proteins. The stabilization of existing proteins is an important factor in minimizing protein turnover, as such it is expected that this term should be upregulated. Indeed, previous studies have shown that red-eared slider turtles have increased liver heat 
shock protein expression under anoxic conditions, which is crucial in the protection of protein integrity to prevent their degradation (Kesaraju et al., 2014; Krivoruchko \& Storey, 2010d; Prentice et al., 2004; Stecyk et al., 2012). The prediction of decreased unfolded protein binding could perhaps be the result of downregulating other genes involved in this GO MF term that are not critical to anoxia survival or are redundant in order to conserve cellular energy. Future studies should be done to analyze the expression of additional proteins involved in binding unfold proteins to support or refute these predictions. Despite this potentially contradictory finding, there is an overall suppression of protein turnover predicted in anoxia, which most likely functions to extend the lifespan of already translated proteins as a mechanism for energy conservation in the hypometabolic state.

\subsubsection{Olfactory receptors and stimulus detection}

Significant upregulation of the KEGG olfactory transduction pathway (Figure 3.6) and GO MF olfactory receptor activity (Figure 3.5) during anoxia as indicated by their positive model coefficients is interesting given that it is not commonly thought to be associated with the liver. However, studies have shown that the liver and other non-sensory organs do in fact utilize olfactory receptors (ORs) to initiate signal transduction triggered by the chemosensory function of these receptors (Kurtz et al., 2020; Maßberg \& Hatt, 2018). Ectopically expressed ORs are G protein-coupled receptors that detect chemical stimuli in the environment and activate various signaling cascades, depending on the specific identity of the OR triggered (Maßberg \& Hatt, 2018). These ORs can activate a wide variety of molecular mechanisms within the cell including cell proliferation, growth, differentiation, migration, invasiveness, apoptosis, chemotaxis, angiogenesis, and hepatic metabolism among others (Kang \& Koo, 2012; Kurtz et al., 2020; Maßberg \& Hatt, 2018). 
Given that the liver is a crucial metabolic organ that regulates blood chemical levels, it holds that liver would use these ORs to sense stimuli and control the subsequent biochemical response. This may be especially important during anoxia when cellular conditions are rapidly changing, and other organs are dependent on liver glycogenolysis to produce the glucose they need to maintain adequate glycolytic function (Kurtz et al., 2020). Curiously, many of the pathways activated by OR stimulation are related to cancer, which had multiple KEGG pathways (prostate cancer, pathways in cancer, and small cell lung cancer) downregulated in anoxia (Figure 3.6). This downregulation may serve as a countermeasure to prevent the concomitant activation of deleterious and energetically expensive processes associated with the major hallmarks of cancer, leaving only the critical anoxia-responsive pathways to be activated by OR signal transduction. Liver olfactory function likely ties in with the GO BP pathways sensory perception of chemical stimulus, sensory perception, and detection of stimulus (Figure 3.4), and GO MF G protein-coupled receptor activity (Figure 3.3) that are also upregulated during anoxia. Together, these pathways are likely responsible for hepatic detection of environmental stimuli and serve to activate the appropriate signaling cascades that regulate the liver's detoxification and glycogenolytic functions. Further studies will need to be done to confirm the exact role ORs and sensory detection pathways serve in red-eared slider turtle liver during anoxia, as well as the downstream effects of this chemosensory mechanism.

\subsubsection{Ribosomal regulation of translation}

Another interesting finding was the predicted upregulation of ribosome associated pathway terms during anoxia. GO MF analysis indicated that the structural constituent of ribosome term was upregulated (Figure 3.3), while GO CC predicted upregulation of many 
ribosome terms including ribosomal subunit, cytosolic ribosome, ribosome, and cytosolic large ribosomal subunit (Figure 3.5). KEGG gene set analysis also showed a positive model coefficient for the ribosome pathway (Figure 3.6). All of these results suggest that there is less miRNA-mediated silencing of the ribosome mRNA transcripts during anoxia leading to increased ribosome expression. This is intriguing as one would expect ribosome expression to be downregulated due to the high energetic cost of protein translation. In fact, protein turnover accounts for over $50 \%$ of ATP usage in normoxic turtle hepatocytes (Hochachka \& Lutz, 2001). Thus, protein translation should theoretically be suppressed to conserve cellular energy during anoxia when red-eared slider turtles have a limited capacity to produce ATP.

However, another study found that red-eared slider liver likely either maintains normoxic levels of translation or may even upregulate it in response to anoxic stress (Szereszewski \& Storey, 2018). This translation capacity could serve to support key anoxia-survival responses such as enhanced antioxidant defences, chaperone proteins, and various other cytoprotective factors during anoxia. Additionally, the maintenance of translation may aid critical metabolic functions such as glycogenolysis and glucose metabolism (Szereszewski \& Storey, 2018). Having an increased capacity to catabolize glycogen for both internal energy production and/or for export as a fuel to other organs would be crucial to long-term survival of the anoxic stress. While these ideas are interesting, certain GO terms associated with protein translation including tRNA transport (GO BP; Figure 3.4) and endoplasmic reticulum (GO CC; Figure 3.5) had negative model coefficients and thus were predicted to have increased negative regulation via miRNAs implying that while the ribosome and its subunits were upregulated, translation may be 
impaired. These conflicting findings demonstrate the need for further study to better understand the hepatic translational landscape during anoxia.

\subsection{CONCLUSIONS}

This study presents the first omics-based analysis of miRNA expression as a response to anoxia in the red-eared slider turtle using small RNA sequencing techniques. The results described herein demonstrate that a total of 13 miRNAs were downregulated and 10 miRNAs were upregulated during anoxia. Additionally, bioinformatic analyses predicted that pathways associated with cell cycle control and protein modification and protein turnover would be significantly downregulated as a result of this miRNA expression profile. This supports the findings of previous studies which identified cell proliferation is a key target of miRNA repression. Interestingly, pathways associated with stimulus detection and ribosome expression appear to be upregulated in response to anoxia. These pathways should be analyzed further to elucidate the exact effects that miRNAs have on these processes. Overall, this study identified miRNAs that are involved in regulating the liver's metabolic function and suppression of nonessential, energy-intensive processes. This miRNA-mediated hepatoprotective mechanism likely acts to prevent liver degradation during MRD to maintain its detoxification and glycogenolytic activity. Taken together, these findings point towards a reliance on liver functionality during anoxia, meaning that the red-eared slider turtle must maintain a significant level of hepatic activity even in a hypometabolic state. 


\subsection{FIGURES}

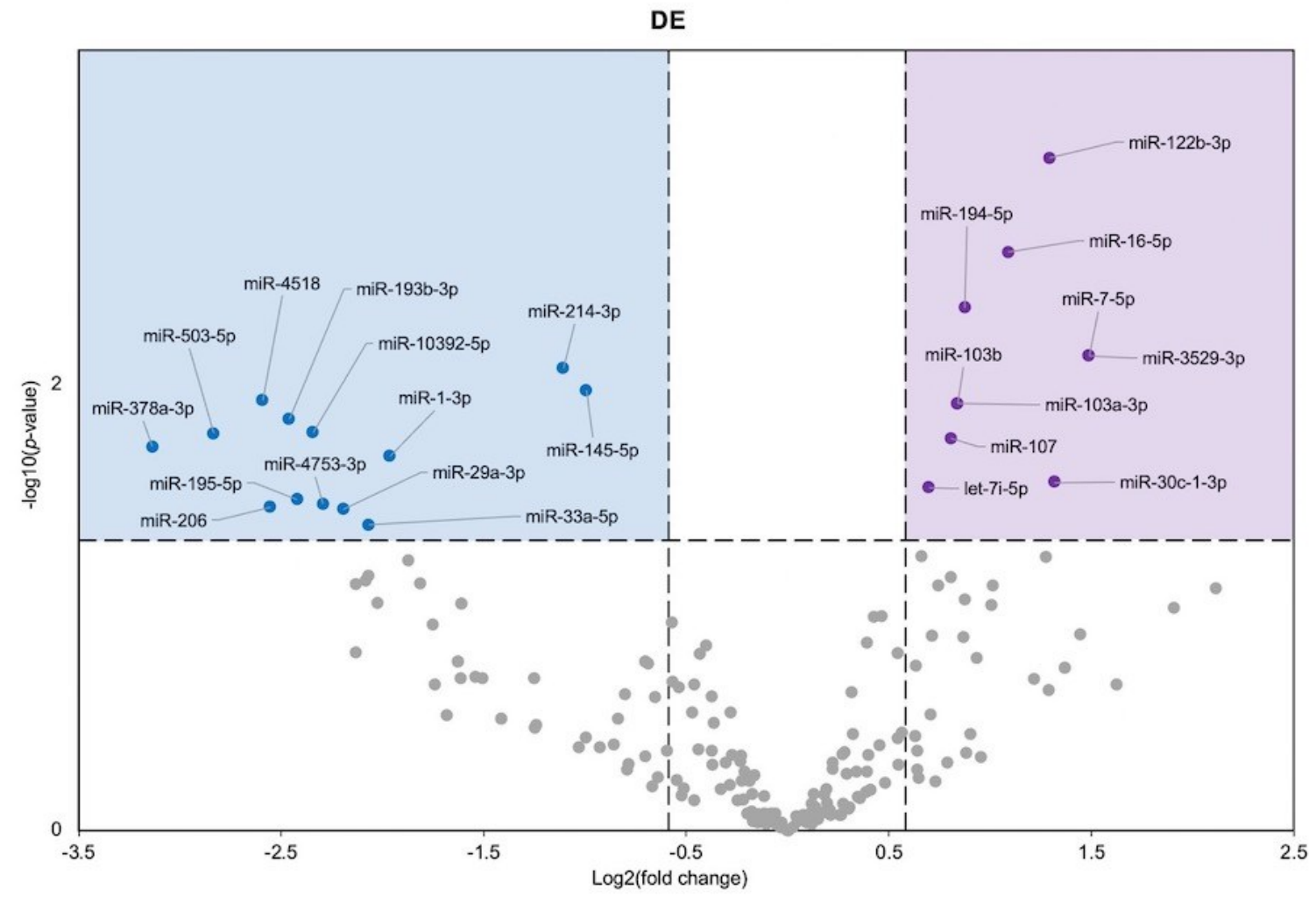

Figure 3.1. MicroRNA differential expression (DE) analysis in the liver of Trachemys scripta elegans in response to $20 \mathrm{~h}$ anoxic exposure. Volcano plot fold-change thresholds were set to $\pm \log _{2} 1.5$ and $-\log _{10}(p$-value), with a false discovery rate (FDR)-adjusted $p<$ 0.05. Blue circles represent statistically significant downregulation and purple circles indicate miRNA that were significantly upregulated. Grey circles are miRNA that did not pass the fold-change and $p$-value thresholds. 


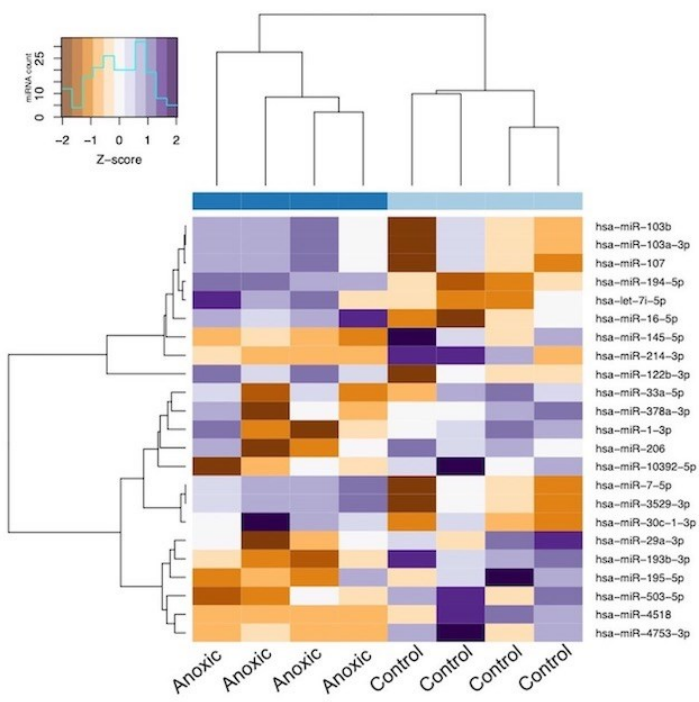

B

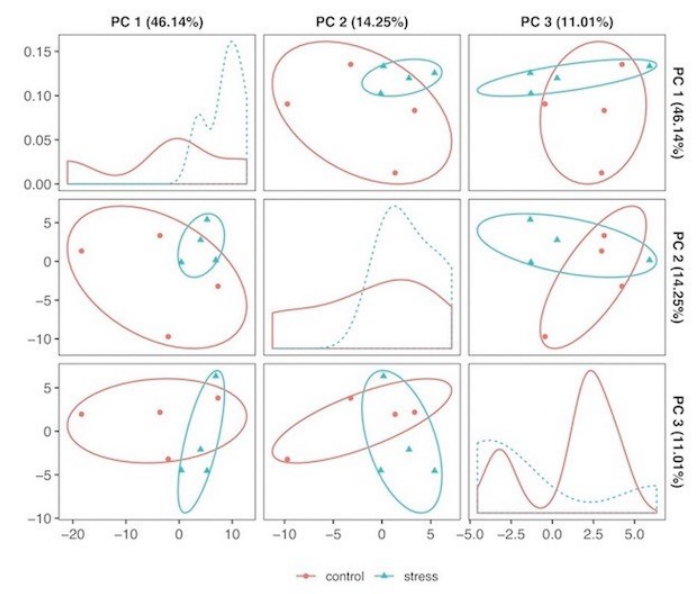

Figure 3.2. Unsupervised clustering analyses in of miRNA species in liver of aerobic control vs $20 \mathrm{~h}$ anoxia exposed Trachemys scripta elegans. (A) Hierarchical clustering heatmap and dendrograms for significantly differentially expressed miRNA. Light and dark blue bars represent the two clusters that the samples were divided into based on their miRNA expression patterns. Purple and orange indicate higher and lower z-scores (standardized expression level) for each miRNA in each sample, respectively. (B) Principal component analysis (PCA) on $\mathrm{PC} 1, \mathrm{PC} 2$, and $\mathrm{PC} 3$, with density plots for samples and miRNA scores on the diagonal (top left to bottom right). 


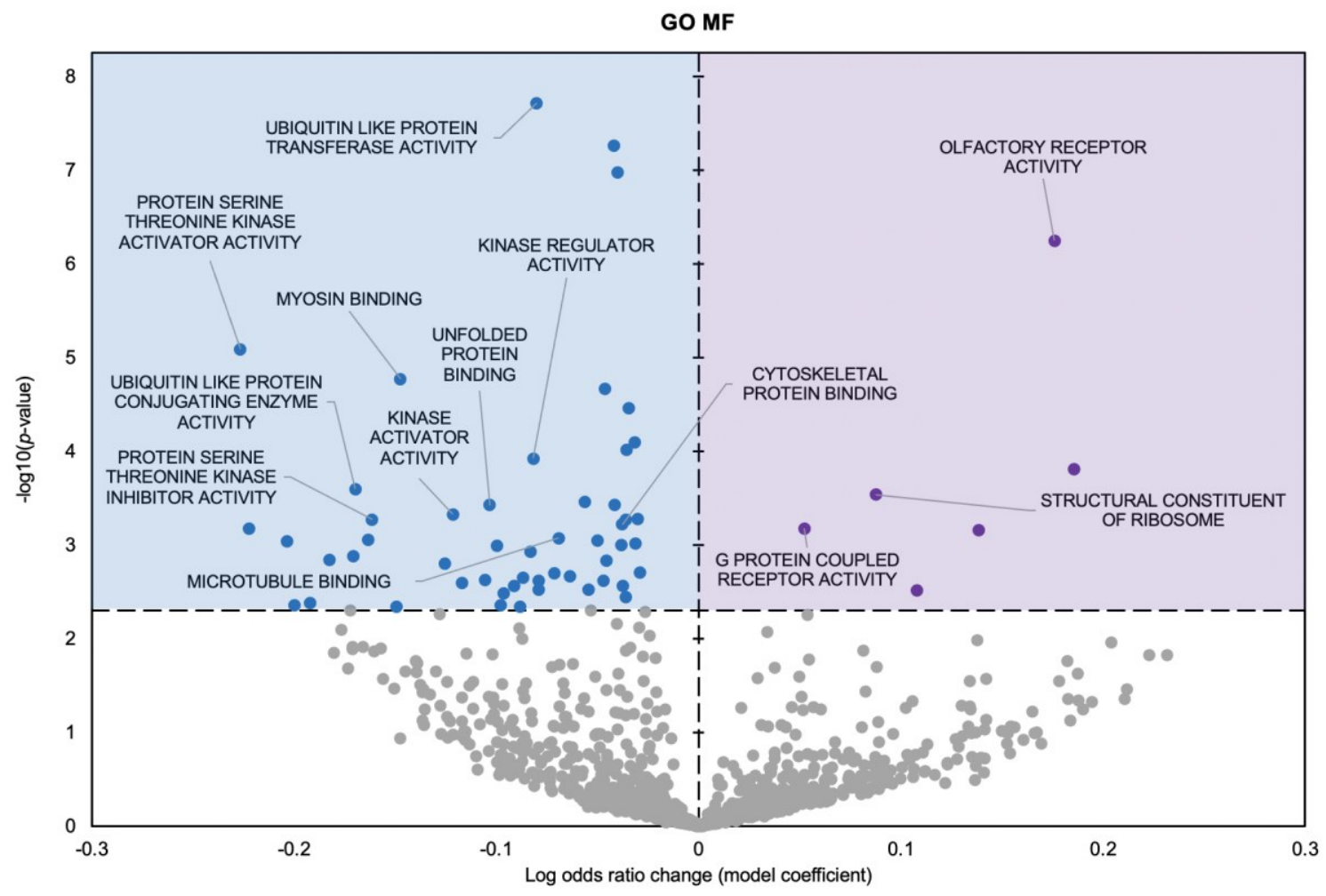

Figure 3.3. GO molecular function (MF) term gene set enrichment based on miRNAs identified in DE analysis of liver from anoxic Trachemys scripta elegans relative to controls. Volcano plots show term enrichment with an FDR-adjusted $p$-value $<0.05$ and fold change of $>1.5$. Blue circles indicate significantly enriched terms with negative model coefficients (more miRNA-inhibited; decreased expression), and purple circles indicate significantly enriched terms with positive model coefficients (less miRNA-inhibited; increased expression). 


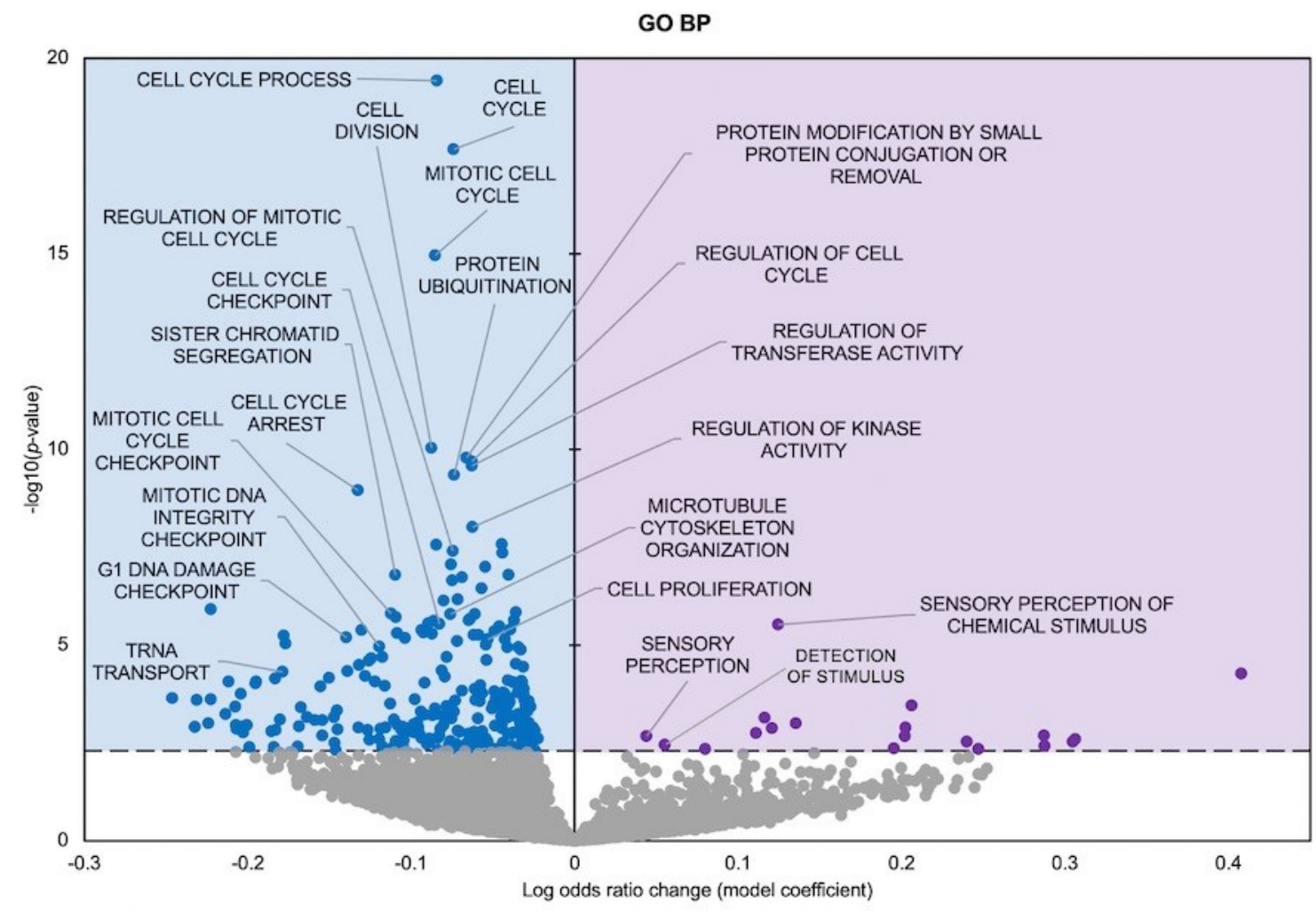

Figure 3.4. GO biological process (BP) term gene set enrichment based on miRNAs identified in DE analysis of liver from anoxic Trachemys scripta elegans relative to controls. All other information as in Figure 3.3. 


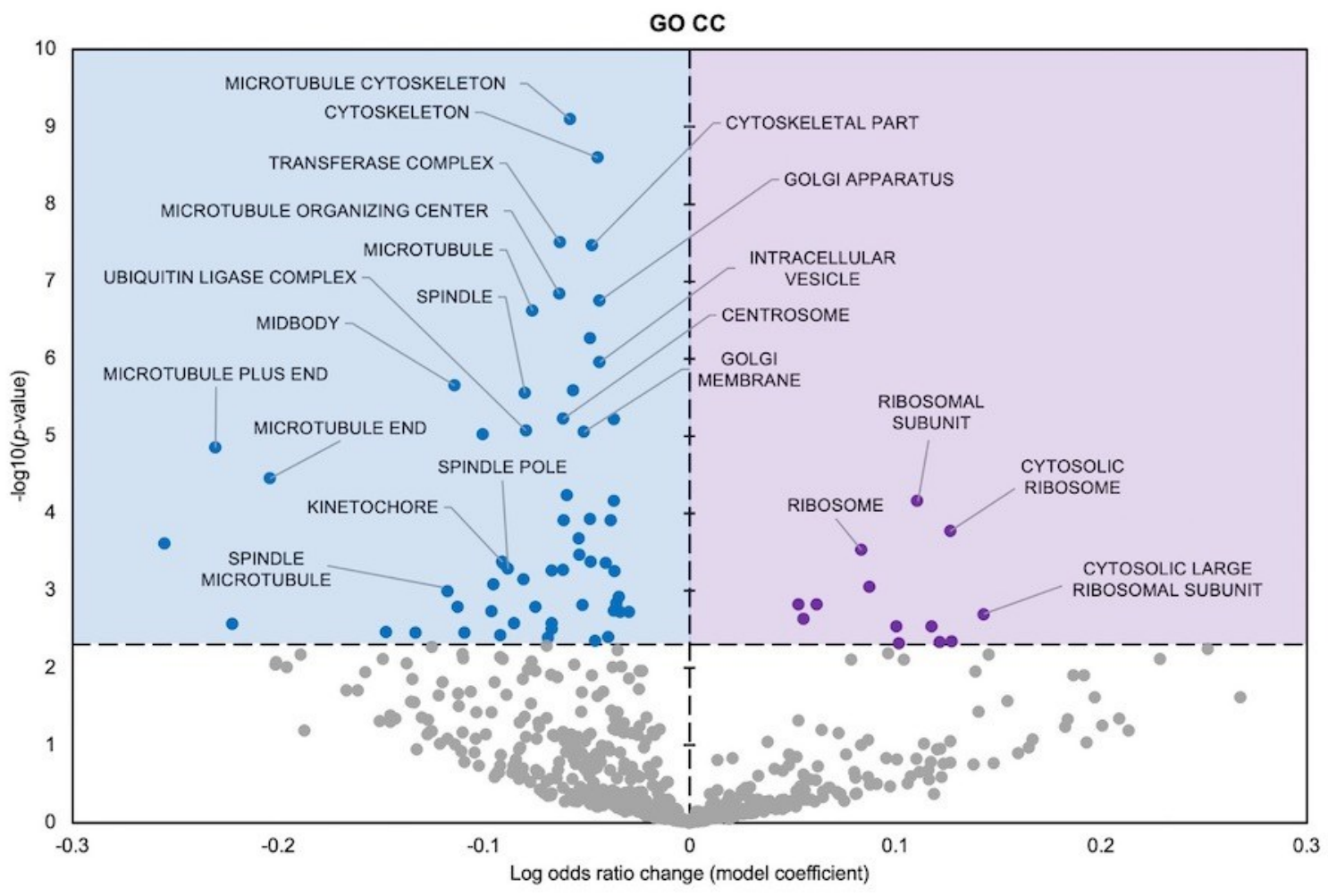

Figure 3.5. GO cellular component (CC) term gene set enrichment based on miRNAs identified in DE analysis of liver from anoxic Trachemys scripta elegans relative to controls. All other information as in Figure 3.3. 


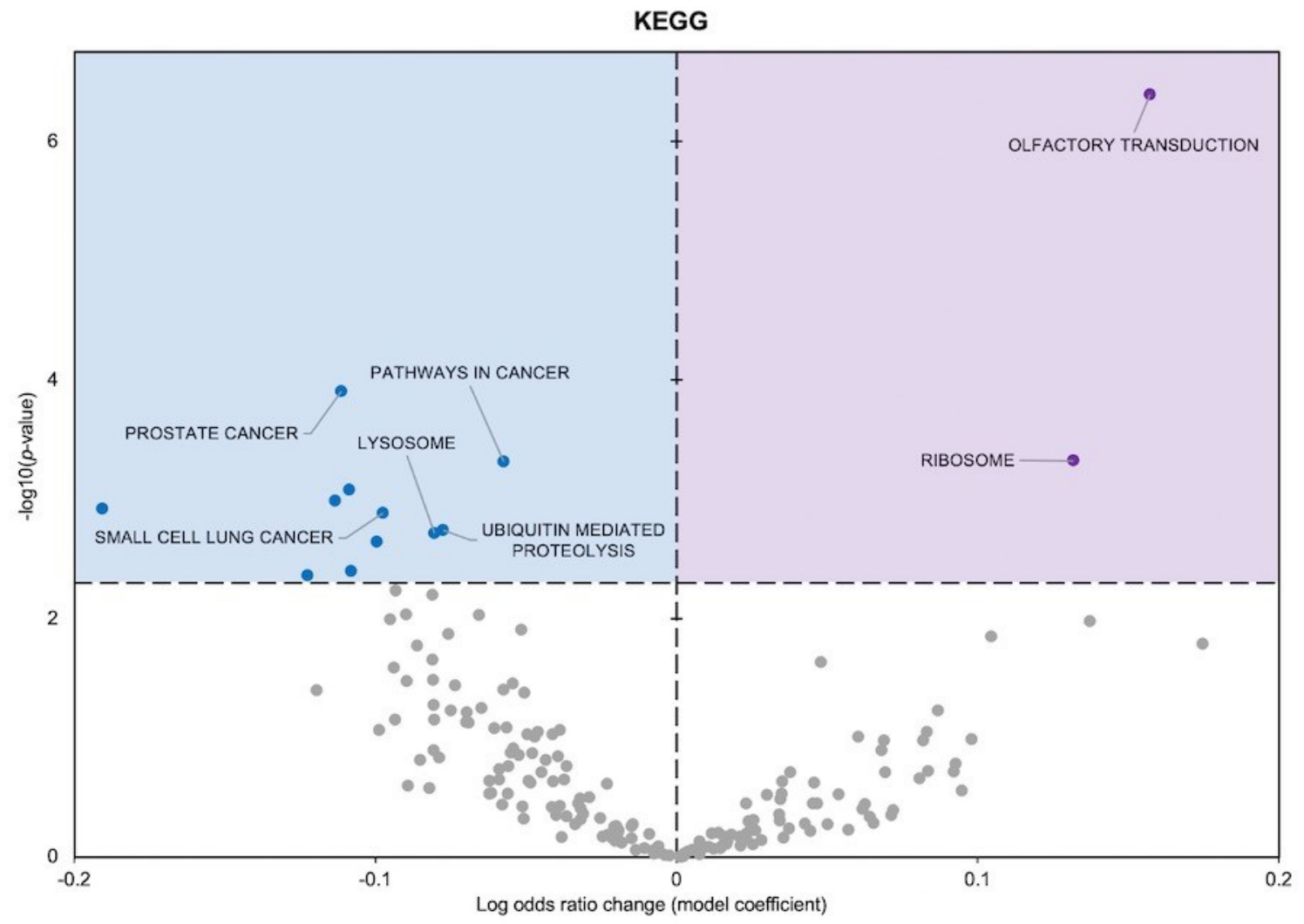

Figure 3.6. KEGG pathway term gene set enrichment based on miRNAs identified in DE analysis in liver from anoxic Trachemys scripta elegans relative to controls. All other information as in Figure 3.3. 


\section{CHAPTER 4 General Discussion}




\subsection{Red-eared slider turtles and anoxia}

Many animals are naturally equipped to survive period of harsh environmental stress far beyond what other species can endure. These stresses can take numerous forms, including extreme hot or cold temperatures, lack of food or water, or limited oxygen availability. Oxygen deprivation is a widespread environmental stress many animals face either due to behavioural traits that temporarily interrupt their oxygen supply (e.g. aerial exposure of gill-breathers, breath-hold diving of lung-breathers) or natural variations in environmental oxygen availability (e.g. altitude changes, oxygen depletion in water or sediments, bodies of water becoming ice-locked, whole body freezing) (Storey, 1996). Although brief exposure to hypoxic/anoxic conditions is normally tolerated relatively well by most animals, prolonged exposure is often lethal. This is due to the normal dependence by most animals on aerobic metabolism for the production of the ATP necessary for their long-term survival. When the supply of oxygen is disrupted, anaerobic glycolysis becomes the sole source of cellular energy and most animals are not able to survive on this reduced ATP yield. However, the red-eared slider turtle is one of the best facultative anaerobes among terrestrial vertebrates and is well equipped to survive using anaerobic metabolism alone (Storey, 2007).

In order to endure prolonged bouts of oxygen limitations, red-eared sliders have developed numerous physiological and biochemical adaptations that allow them to overcome the cellular consequences of anaerobic glycolysis. During winter, these turtles hibernate at the bottom of ponds and lakes that can often become ice-covered that can make it impossible for the animals to rise to the surface to breathe. Some turtle species can employ extrapulmonary oxygen uptake but hard-shell turtles like $T$. $s$. elegans are relatively 
poor at this (Jackson \& Ultsch, 2010; Storey, 2007). As such, they have additional biochemical mechanisms that enable them deal with the buildup of toxic fermentative end products (lactic acid) and prevent cellular damage while reducing their overall metabolic needs (Storey, 2007). Firstly, the excess protons and lactate anions that are produced from glycolysis are buffered by bicarbonate anions and $\mathrm{Ca}^{2+}$ cations released by the shell and skeleton and lactate is stored into the shell; this prevents lethal lactic acidosis (Jackson, 2000). Simultaneously, multiple protective mechanisms such as enhanced antioxidant defences and stress-activated chaperone proteins are leveraged to prevent damage to macromolecules as a result of cellular insults and a changing intracellular environment (Breedon et al., 2021; Krivoruchko \& Storey, 2010c, 2010d; Willmore \& Storey, 1997a). In addition to these protections, the turtles must contend with little or no opportunity to feed and, hence, the metabolism is based mainly on large reserves glycogen, particularly in the liver, that can be mobilized as fuel (Jackson \& Ultsch, 2010). This is crucial to their survival because if there is a complete shutdown in ATP synthesis, the turtle will die as it would no longer be able to maintain critical life-sustaining processes.

In order to slow the rate at which internal fuel reserves are depleted and to maintain balanced cellular energetics (i.e. energy production vs. energy consumption), red-eared slider turtles need to significantly decrease their metabolic rate, suppress many cellular activities, and enhance key pro-survival pathways (Storey, 2007). A reduction in body temperature associated with the drop in ambient water temperature serves to decrease the turtle's metabolic rate that, when combined with various biochemical mechanisms, results in their metabolism being reduced by $\sim 90 \%$ (Hochachka, 1988; Jackson, 1968; Storey, 2007). Molecular regulation of metabolism occurs at every level to facilitate MRD. These 
mechanisms lead to greatly reduced fuel use and energy consumption to a level that is able to be met by the anaerobic glycolysis alone. Whilst in the hypometabolic state, cellular energy is diverted towards powering pro-survival pathways. The selective expression of certain processes ensures that they are able to maintain critical cellular functions that must be retained even in a stress state and activate anoxia-specific responses, both of which are needed for the turtle to survive long-term.

Given their incredible ability to endure prolonged oxygen deprivation, red-eared sliders are an ideal model organism to expand our understanding of the molecular underpinnings of natural anoxia tolerance. In particular, elucidating how they can so proficiently reduce their metabolic rate to enter hypometabolic state could have important implications in human medical research (see Section 4.4.). In their quest to achieve MRD and meet the energy needs of vital cell functions, these turtles utilize various transcriptional, post-transcriptional, translational, and post-translational regulatory mechanisms (Biggar \& Storey, 2011; Storey, 2007). As such, the various regulatory mechanisms utilized by turtles to achieve MRD are a crucial area of study. Notably, posttranscriptional regulation through miRNA-mediated mRNA suppression has proven to be a tremendous tool used by many stress-tolerant organisms in the pursuit of MRD and metabolic reorganization.

\subsection{Role of microRNAs in the stress response to anoxia}

The use of miRNAs for the post-transcriptional silencing of mRNA transcripts is advantageous, especially during periods of MRD and metabolic reorganization. In response to stress, miRNA action can allow an organism to quickly and reversibly alter their gene expression in a tissue-specific manner. This dynamic regulation enables the expression of 
pro-survival genes while simultaneously supressing the expression of nonessential genes, which ultimately serves to conserve vital cellular energy that is severely limited under anaerobic conditions. Moreover, the use of miRNA-mediated gene silencing is utilized in the stress response of many organisms, as seen by the numerous studies conducted on a wide variety of animals including frogs, snails, squid, crayfish, insects, ground squirrels, lemurs, naked mole rats, bats, and bears (Biggar \& Storey, 2014; English et al., 2018; HadjMoussa et al., 2018, 2020, 2021; Hawkins \& Storey, 2020; Hoyeck et al., 2019; Logan \& Storey, 2021; Luu et al., 2020; Lyons et al., 2016). As such, it is worthwhile to analyze miRNA regulation and expression in the red-eared slider turtle, as it is one of the vertebrates best adapted to survive prolonged anoxic episodes. The expression of a select few miRNAs was previously measured, and their differential expression was found to mainly target cell proliferation and metabolic processes (Biggar \& Storey, 2012, 2017; Zhang et al., 2013). These studies provided an excellent starting point, but a more thorough study of the red-eared slider turtle's miRNA response, as performed in this thesis, provides us with a better understanding of the molecular mechanisms underlying their anoxia tolerance.

In Chapter 2 of this thesis, miRNA biogenesis and mRNA fate were examined in the liver and red muscle of normoxic, anoxic, and reoxygenated turtles via immunoblotting to determine how miRNA expression is regulated and what effects this may have on mRNA transcripts. Based on previous research, it was expected that there would be tissue-specific expression of proteins associated with miRNA biogenesis and mRNA fate in response to anoxia and aerobic recovery. Indeed, there was an observed difference in the miRNA silencing response between organs, supporting the idea that because miRNA expression is 
tissue-specific, miRNA biogenesis and the resulting mRNA fate is also tissue-specific. The expression pattern of biogenesis proteins in the liver provided two main theories: (1) overall downregulation of miRNA synthesis as a result of decreased nuclear processing, or (2) cytoplasmic stores of pre-miRNA built up during early anoxia exposure are sufficient to support adequate miRNA expression. Muscle biogenesis proteins, on the other hand, showed increased expression of many processing proteins, particularly during reoxygenation, suggesting that miRNAs may act as key regulators of gene expression in the transition back to aerobic metabolism. These results corroborate the hypothesis that biogenesis would be tissue specific, likely in order to support different levels of organ function during anoxia and aerobic recovery. Based on the findings in a study on miRNA biogenesis in the brain of frozen wood frogs, the miRNA response also appears to be a species-specific and/or stress-specific (Hadj-Moussa \& Storey, 2018). That study identified downregulation in multiple miRNA processing factors in response to freezing stress and subsequent thawing (Hadj-Moussa \& Storey, 2018). The pattern of suppressing early nuclear processing stages is shared with the liver of red-eared slider turtles, but biogenesis in wood frogs appears to be suppressed more evenly across the entire process (Hadj-Moussa \& Storey, 2018). Future studies on red-eared slider brains could shed light on whether or not this pattern of biogenesis downregulation is actually species-specific, or if it is a conserved response in the brain of stress-tolerant animals. Additionally, the brief analysis of cytoplasmic granules in Chapter $\mathbf{2}$ indicated that liver likely utilizes P-bodies, whereas muscle showed preference for stress granules as sites for mRNA silencing. Although intriguing, these results are far from conclusive and further studies are needed to confirm their validity. Overall, the results in Chapter 2 indicate that dynamic tissue- 
specific regulation of miRNA biogenesis and translational silencing of mRNAs is crucial for MRD and long-term anoxia survival.

Chapter 3 built on the analysis of miRNA regulation by examining the liver miRNAome using small RNA sequencing and bioinformatic techniques to determine miRNA expression during anoxia and predict the downstream effects of this expression profile. The results showed that there was no overwhelming up- or down-regulation of miRNA expression, but instead there was a dynamic balance of enhanced (10 miRNAs) and suppressed (13 miRNAs) expression. Still, this mixed expression profile led to an overall significant suppression of multiple proteins associated with numerous GO and KEGG terms. Based on previous research, it was expected that miRNA expression would change during anoxia, that metabolically demanding processes such as cell cycle/proliferation would be downregulated in order to conserve energy, and that prosurvival pathways would be upregulated. Indeed, cell cycle processes were predicted to be heavily downregulated during anoxia, as was protein modification and turnover, which is also known to be energetically costly. Interestingly, upregulation was observed in stimulus detection and ribosome terms, implying that the liver's ability to sense and respond to environmental stimuli with appropriate protein expression is critical for anoxia survival. Overall, the sequencing data and bioinformatic analyses suggests that miRNA expression is very important for regulating MRD and pro-survival processes, a finding which is shared across species and stresses.

Taken together, the findings in this thesis support previous research on tissuespecific miRNA regulation, expression, and mRNA silencing in other stress tolerant species. Turtle liver metabolism is suppressed to a certain degree but not completely, 
probably due to the role of liver in regulating glucose fuel output from liver glycogen reserves to serve both internal and external organ ATP production, as well as maintaining its role in detoxification. While needing to conserve energy, the liver also has to express key hepatoprotective mechanisms that enable it to continue in these functions. Posttranscriptional gene silencing via differential miRNA expression provides an excellent tool for turtles to utilize to enact the selective expression of certain processes while suppressing other nonessential genes. As such, the dynamic expression of miRNA necessitates that miRNA biogenesis and mRNA suppression be equally dynamic in order to produce adequate amounts of miRNA to selectively silence protein translation, but not produce too much that it consumes excess ATP. Overall, this study is the first to analyze miRNA biogenesis and the complete miRNAome in anoxia-tolerant red-eared slider turtles and provides valuable avenues for future study. These findings illustrate how miRNA expression is leveraged to regulate mRNA silencing and control the expression of downstream cellular processes.

\subsection{Future directions}

There are numerous avenues for future research to expand on previous studies and the results presented in this thesis. To start, the most obvious first step would be to perform miRNA-seq analyses on liver from turtles during/after aerobic recovery. A comparable analysis of muscle miRNA responses to anoxia and aerobic recovery would also provide a comparison for better understanding of both liver and muscle responses to anoxia. Future studies using RT-qPCR would also need to be done to validate the miRNA sequencing findings presented in Chapter $\mathbf{3}$ by measuring the expression of select differentially expressed miRNAs. Beyond the two tissues analyzed in this thesis, performing the analyses 
from Chapter $\mathbf{2}$ and Chapter $\mathbf{3}$ on additional organs (brain, kidney, etc.) would explore exactly how tissue-specific miRNA regulation and expression is in the turtle. This would reveal whether miRNA biogenesis is regulated at the same steps or if alternative steps are targeted in a tissue-specific manner, and how mRNA is regulated in different critical organs. Additionally, miRNA sequencing and bioinformatic analyses would indicate whether different organs display similar miRNA expression profiles and whether there are shared gene set enrichment targets. Another interesting future direction would be to perform the analyses from Chapter $\mathbf{2}$ and Chapter $\mathbf{3}$ with multiple sampling points over a prolonged time course, starting from initial underwater submergence where turtles are hypoxic and sampling regularly until the turtles have reached full anoxia, followed by periodic sampling as oxygen is reintroduced. This could provide greater insight into the order that anoxia-responsive processes are regulated and the timing at which they are expressed throughout anoxic exposure.

While there is a near endless number of projects that could be undertaken to expand the scope of this thesis, I believe there are three crucial research areas that would provide the most insight into the role played by miRNA in achieving anoxia-tolerance for the redeared slider turtle: (1) upstream analyses of miRNA transcription, (2) comprehensive analyses of mRNA fate, and (3) downstream analyses of enriched pathways and processes. Additionally, studies could also be undertaken to expand the research scope beyond miRNA-based regulation of gene expression.

\subsubsection{Controlling microRNA transcription}

Beyond miRNA biogenesis and maturation as discussed in Chapter 2, miRNA expression is also regulated by the rate of pri-miRNA transcription. Thus, studies analyzing 
transcription factors that initiate miRNA transcription would be an enlightening territory to expand into. Transcription factors work by acting on regulatory sequences within the DNA to modulate transcription, resulting in increased or decreased transcript abundance. These proteins have been previously implicated as a critical regulator of gene expression in response to environmental stress in many stress-tolerant organisms, thus making them an ideal target for new research (Krivoruchko \& Storey, 2015). Future studies could use numerous techniques such as immunoblotting and kit-based assays, among others, to assess the expression, subcellular localization, protein modification status, and activity of selected transcription factors, as well an analysis of the various co-activators/binding partners. Transcription factors of interest would be chosen according to the miRNAs differentially regulated in Chapter 3 to provide the most relevant knowledge relating to the regulation and expression of miRNA during anoxia. These studies would provide an interesting look at how transcription factors interact with the downstream biogenesis pathway to fine-tune miRNA production in response to extended bouts of anoxia and aerobic recovery.

\subsubsection{Messenger RNA fate following microRNA targeting}

Chapter 2 also raises the need to perform a more expansive study into stress granule and P-body formation and function during anoxia and aerobic recovery. The fate of mRNA was limited to a preliminary analysis of a few key proteins; however, there are numerous other proteins that also contribute to stress granules/P-bodies, which remain to explored. These proteins could be analyzed through immunoblotting of total cellular and subcellular fractionate protein samples to also provide a general idea of where these storage bodies are localizing during stress. However, a more intensive and accurate approach to analyzing mRNP granules would be to use microscopy techniques to actually visualize 
stress granules and P-bodies and assess the size and/or number formed during anoxia/reoxygenation. Additionally, these granules can be isolated so as to extract the mRNA they contain, and then this mRNA can be sequenced to unveil the mRNA species that are being targeted to stress granules/P-bodies for translational suppression (Hubstenberger et al., 2017). Alternately, the miRNAs identified from Chapter 3 could be used to indicate potential mRNA targets, which could then be analyzed through qPCR to quantify their expression and determine how they are altered by the anoxia-induced differential miRNA expression. Immunoblotting can be used to further expand on the qPCR data by measuring the expression of the proteins that these mRNAs encode to see if the mRNAs present are translationally silent.

\subsubsection{Effect of microRNA regulation on downstream pathways}

All of the cellular processes and pathways that were predicted to be most affected by miRNA regulation in Chapter 3 warrant further investigation to determine the probable effects that the differentially expressed miRNAs have on these processes. These pathways can be analyzed in a manner similar to how miRNA biogenesis was analyzed in Chapter 2, i.e. through immunoblotting of proteins involved in the pathway, as well as pathway end products. Immunoblot data would provide insight into how miRNA targeting affects the function of these pathways. Immunoblotting experiments could be supplemented by qPCR analyses of the expression of relevant mRNAs, which would allow us to see how both transcript and protein levels change as a result of miRNA targeting. In terms of the processes predicted in Chapter 3, the cell cycle and certain protein modifiers and ribosomal factors have been previously studied in some detail (Biggar \& Storey, 2012; Ding et al., 2019; Greenway \& Storey, 2000; Mattice et al., 2018; Zhang et al., 2013). 
However, many areas remain understudied, especially the role of olfactory receptors and stimulus detection, which would make excellent targets for follow-up research.

\subsubsection{Beyond microRNA regulation}

miRNA would not act in isolation to achieve MRD and there are multiple other mechanisms by which organisms can regulate which genes are expressed during the stress response and to what extent. Thus, there are many avenues for future research into the regulation of gene expression beyond miRNAs including various epigenetic mechanisms, which collectively comprise a major part. Epigenetic regulation mediates gene expression through reversible modifications that do not alter the DNA sequence, making this form of regulation a powerful tool to achieving stress-specific gene expression. Epigenetic controls take various forms and are undoubtedly involved in achieving and maintaining MRD in anoxia tolerant species. To date, epigenetic research on red-eared sliders has focused on mainly on DNA methylation, histone acetylation, and histone methylation, that overall point towards decreased global gene expression (Krivoruchko \& Storey, 2010a; Wijenayake et al., 2018; Wijenayake \& Storey, 2016, 2020). However, epigenetics is a vast field and there is much that has yet to be studied including RNA modifications, particularly the most prominent mRNA mark, $\mathrm{m}^{6} \mathrm{~A}$, which could be an interesting complement to this study of mRNA silencing (N. Liu \& Pan, 2015). All of these are important targets for future research, and could be analyzed through immunoblotting, activity assays, and kits designed to quantify the amount of each posttranslational modification as in the aforementioned turtle papers (Krivoruchko \& Storey, 2010a; Wijenayake et al., 2018; Wijenayake \& Storey, 2016, 2020). Expanding future studies into these and other forms of epigenetic regulation in the red-eared slider turtle will further our understanding of anoxia-induced 
gene expression and how it ties into post-transcriptional miRNA gene silencing as explored in this thesis.

\subsection{Significance and potential applications}

Research into the natural anoxia-tolerance of the red-eared slider turtle has multiple implications for better understanding of some human health and medical concerns. Analysis of the various molecular mechanisms that enable these remarkable animals to survive prolonged periods of oxygen deprivation could be a key to developing novel medical interventions to deal with the effects of hypoxia/ischemia in human patients experiencing stroke, heart attack or other conditions. In particular, miRNAs are of major interest and are currently being studied in relation to their role as biomarkers in various cancers, infectious diseases, neurological and neurodegenerative disorders, among others (Hammond, 2015; Montano, 2011; Walayat et al., 2019). Through miRNA studies in anoxia-tolerant and other stress-tolerant animals, we can identify miRNAs that are crucial for basic cell survival, as well as miRNAs that help facilitate a hypometabolic state or enhance cell protection mechanisms during a stress event. These miRNAs can then be used as both biomarkers and as treatments to help induce a beneficial stress response. For example, if we knew that a particular subset of miRNAs act as hepatoprotectants, these miRNAs could be exogenously administered to protect the liver against further insult or maybe even reverse existing injuries.

Beyond their role as biomarkers for disease, miRNA function in MRD could have important applications in organ preservation and transplantation, and the prevention of organ IRI caused by severe medical events such as cardiac arrest, stroke, and traumatic injury (Giwa et al., 2017; Hadj-Moussa \& Storey, 2019; Stancic et al., 2018). In humans, 
lack of oxygen leads to organ damage that can rapidly become irreversible or even fatal if oxygen/blood flow isn't quickly restored, and even then, the sudden reperfusion of blood and return of oxygen to the tissue may result in additional oxidative damage. Prolonged anoxic episodes experienced by the red-eared slider turtle parallel the oxygen deprivation experienced by human organs following severe medical events with one crucial difference: the turtles are able to survive for months without suffering any apparent injuries. As such, understanding how organisms such as the red-eared slider turtle can reduce their metabolic rate and survive repeated cycles of prolonged anoxia and reoxygenation through miRNA and other molecular mechanisms is critical to gaining a better understanding of human organ damage associated with oxygen deprivation and how to mitigate or reverse such damage.

Current organ preservation techniques mainly rely on static cold storage whereby organs are placed on ice to reduce their metabolic rate and keep them from decaying (Bellini \& D'Andrea, 2019; Salehi et al., 2018). This helps to minimize damage due ischemia from lack of oxygen, loss of blood circulation and accumulation of metabolic end products. However, cold temperatures can also lead to cellular damage and, hence, hypothermic storage is not a feasible solution for maintaining organ viability beyond a few hours. Therefore, supplementing traditional cold storage with novel techniques based on MRD and pro-survival mechanisms as seen in red-eared sliders could be applied to increase the length of time that organs removed for transplant could remain viable when stored on ice. Moreover, mimicking the physiological conditions of the hibernating turtle in human tissues could provide even better organ preservation outcomes. For instance, if organs are cooled to around $\sim 3^{\circ} \mathrm{C}$ and the various anoxia induced MRD and pro-survival mechanisms 
turtles utilize could be exogenously expressed, then the viability window could be significantly expanded. In this manner, anoxia-tolerant turtles could provide a key direction to pursue in the development of new organ preservation techniques. Whether used alongside current preservation techniques or in the development of novel ones, if miRNAs and other regulatory mechanisms used by turtles are applied to help induce hypometabolism and anoxia tolerance in human organs, then they might be able to be preserved and stored outside a living host for longer periods of time before being transplanted. This would extend the viability window for an organ to be transferred from a donor to the recipient, thereby preventing the rampant organ wastage that currently occurs and costs many people their lives while waiting for a transplant.

Additionally, organs can become damaged due to ischemia/blood loss as a result of significant physical injury. In order to combat severe traumatic injury, therapeutic hypothermia has been used to reduce a person's metabolic rate and prevent further damage while the patient is in critical condition. The induction of hypothermia in trauma patients significantly extends the survival window, giving doctors more time to perform any necessary medical treatments to ensure patient survival (Kurisu \& Yenari, 2018; Schenone et al., 2016). The knowledge gained by studying how the red-eared slider turtle is able to dramatically reduce its metabolism, as well as how they are able to endure months with no oxygen could be applied to future technologies that will further improve clinical outcomes of therapeutic hypothermia. If we could take the molecular mechanisms underlying the turtle's incredible anoxia-tolerance/MRD and induce them in humans who have suffered severe physical trauma, we could limit a patient's blood flow to stop them from bleeding out without risking deleterious organ damage and buy valuable treatment time. 
Applications like these would not only prevent ischemic organ damage following medical events but could also reduce the number of blood transfusions needed during surgery and the number of initially viable organs that have "timed-out" and become unsuitable for transplantation, both of which are important because of the current supply shortages. In turn, novel turtle-based medical treatments for ischemia/anoxia survival have the potential to reduce these strains on the medical system, and potentially save many human lives.

\subsection{Summary}

In order to survive on limited or no oxygen, red-eared slider turtles must undergo extensive metabolic suppression and reorganization through the coordinated regulation of various molecular mechanisms. This thesis analyzed the regulation of miRNA expression and its effect on mRNA fate in the red-eared slider turtle (Trachemys scripta elegans) in response to anoxia stress. The data gathered showed that miRNA biogenesis and mRNA fate was highly tissue-specific, with liver and red muscle contrasting each other in the expression of key proteins. In the liver, miRNA biogenesis was shown to be downregulated in early phases during anoxia and recovery but upregulated in later during anoxia, whereas in the muscle, biogenesis was upregulated at multiple phases across the entire process. The miRNA biogenesis findings in liver were expanded by use of miRNA sequencing, which found 10 miRNAs that were upregulated under anoxia, while 13 were downregulated. Additional bioinformatic analyses predicted that this miRNA expression profile would likely lead to suppression of processes associated with the cell cycle and protein modification/turnover under anoxia, whereas other processes could be putatively upregulated due to miRNA downregulation (e.g. environmental stimulus detection, ribosome expression). Interestingly, the occurrence of miRNA downregulation indicates 
that while the red-eared slider turtle needs global suppression of organ metabolic rates, the protective capacity of the liver is likely worth the relatively high energetic cost of upregulating selected key pathways, which would explain why the liver is not entirely metabolically silenced during anoxia. These findings highlight the important role miRNA plays in the regulation of metabolic reorganization during anoxia, providing data that could potentially be utilized in future medical technologies and therapeutics to limit organ damage in humans suffering from hypoxia/anoxia or ischemic stress. 


\section{REFERENCES}


Adams, J. M., Difazio, L. T., Rolandelli, R. H., Luján, J. J., Haskó, G., Csóka, B., Selmeczy, Z., \& Németh, Z. H. (2009). HIF-1: A key mediator in hypoxia (Review). In Acta Physiologica Hungarica (Vol. 96, Issue 1, pp. 19-28). https://doi.org/10.1556/APhysiol.96.2009.1.2

Allis, C. D., \& Jenuwein, T. (2016). The molecular hallmarks of epigenetic control. In Nature Reviews Genetics (Vol. 17, Issue 8, pp. 487-500). https://doi.org/10.1038/nrg.2016.59

Anderson, P., \& Kedersha, N. (2008). Stress granules: the Tao of RNA triage. In Trends in Biochemical Sciences (Vol. 33, Issue 3, pp. 141-150). https://doi.org/10.1016/j.tibs.2007.12.003

Anderson, P., \& Kedersha, N. (2009). RNA granules: Post-transcriptional and epigenetic modulators of gene expression. In Nature Reviews Molecular Cell Biology (Vol. 10, Issue 6, pp. 430-436). https://doi.org/10.1038/nrm2694

Andrews, S. (2010). FastQC: A Quality Control Tool for High Throughput Sequence Data. Babraham Institute. https://www.bioinformatics.babraham.ac.uk/projects/fastqc/

Annunziato, A. T. (2008). DNA Packaging: Nucleosomes and Chromatin. In Nature Education. http://www.nature.com/scitable/topicpage/dna-packaging-nucleosomesand-chromatin-310

Ardekani, A. M., \& Naeini, M. M. (2010). The role of microRNAs in human diseases. In Avicenna Journal of Medical Biotechnology (Vol. 2, Issue 4, pp. 161-179).

Aronesty, E. (2011). Command-line tools for processing biological sequencing data. EaUtils. https://github.com/ExpressionAnalysis/ea-utils

Ashburner, M., Ball, C. A., Blake, J. A., Botstein, D., Butler, H., Cherry, J. M., Davis, A. P., Dolinski, K., Dwight, S. S., Eppig, J. T., Harris, M. A., Hill, D. P., Issel-Tarver, L., Kasarskis, A., Lewis, S., Matese, J. C., Richardson, J. E., Ringwald, M., Rubin, G. M., \& Sherlock, G. (2000). Gene ontology: Tool for the unification of biology. In Nature Genetics (Vol. 25, Issue 1, pp. 25-29). https://doi.org/10.1038/75556 
Attwood, K. M., Robichaud, A., Westhaver, L. P., Castle, E. L., Brandman, D. M., Balgi, A. D., Roberge, M., Colp, P., Croul, S., Kim, I., McCormick, C., Corcoran, J. A., \& Weeks, A. (2020). Raloxifene prevents stress granule dissolution, impairs translational control and promotes cell death during hypoxia in glioblastoma cells. Cell Death and Disease, 11(11). https://doi.org/10.1038/s41419-020-03159-5

Auten, R. L., \& Davis, J. M. (2009). Oxygen toxicity and reactive oxygen species: The devil is in the details. In Pediatric Research (Vol. 66, Issue 2, pp. 121-127). https://doi.org/10.1203/PDR.0b013e3181a9eafb

Bagali, S., A. Hadimani, G., S. Biradar, M., \& K. Das, K. (2018). Introductory Chapter: Primary Concept of Hypoxia and Anoxia. In Hypoxia and Anoxia. https://doi.org/10.5772/intechopen.80270

Bailey, J. R., \& Driedzic, W. R. (1996). Decreased total ventricular and mitochondrial protein synthesis during extended anoxia in turtle heart. American Journal of Physiology - Regulatory Integrative and Comparative Physiology, 271(6 40-6). https://doi.org/10.1152/ajpregu.1996.271.6.r1660

Bansal, S., Luu, B. E., \& Storey, K. B. (2016). MicroRNA regulation in heart and skeletal muscle over the freeze-thaw cycle in the freeze tolerant wood frog. Journal of Comparative Physiology B: Biochemical, Systemic, and Environmental Physiology, 186(2), 229-241. https://doi.org/10.1007/s00360-015-0951-3

Bartel, D. P. (2004). MicroRNAs: Genomics, Biogenesis, Mechanism, and Function. Cell, 116, 281-297. https://doi.org/10.1016/s0092-8674(04)00045-5

Begley, V., Corzo, D., Jordán-Pla, A., Cuevas-Bermúdez, A., Miguel-Jiménez, L. de, Pérez-Aguado, D., Machuca-Ostos, M., Navarro, F., Chávez, M. J., Pérez-Ortín, J. E., \& Chávez, S. (2019). The mRNA degradation factor Xrn1 regulates transcription elongation in parallel to Ccr4. Nucleic Acids Research, 47(18), 9524-9541. https://doi.org/10.1093/nar/gkz660

Behm-Ansmant, I., Rehwinkel, J., \& Izaurralde, E. (2006). MicroRNAs silence gene expression by repressing protein expression and/or by promoting mRNA decay. 
Cold Spring Harbor Symposia on Quantitative Biology, 71, 523-530.

https://doi.org/10.1101/sqb.2006.71.013

Belkin, D. A. (1968). Aquatic respiration and underwater survival of two freshwater turtle species. Respiration Physiology, 4(1), 1-14. https://doi.org/10.1016/00345687(68)90002-9

Bellini, M. I., \& D'Andrea, V. (2019). Organ preservation: which temperature for which organ? In Journal of International Medical Research (Vol. 47, Issue 6, pp. $2323-$ 2325). https://doi.org/10.1177/0300060519833889

Berg, J., Tymoczko, J., \& Stryer, L. (2002). Biochemistry, 5th edition. In Biochemistry.

Biggar, K. K., \& Storey, K. B. (2011). The emerging roles of microRNAs in the molecular responses of metabolic rate depression. In Journal of Molecular Cell Biology (Vol. 3, Issue 3, pp. 167-175). https://doi.org/10.1093/jmcb/mjq045

Biggar, K. K., \& Storey, K. B. (2012). Evidence for cell cycle suppression and microRNA regulation of cyclin D1 during anoxia exposure in turtles. Cell Cycle, 11(9), 1705-1713. https://doi.org/10.4161/cc.19790

Biggar, K. K., \& Storey, K. B. (2014). Identification and expression of microRNA in the brain of hibernating bats, Myotis lucifugus. Gene, 544(1), 67-74. https://doi.org/10.1016/j.gene.2014.04.048

Biggar, K. K., \& Storey, K. B. (2017). Exploration of low temperature microRNA function in an anoxia tolerant vertebrate ectotherm, the red eared slider turtle (Trachemys scripta elegans). Journal of Thermal Biology, 68, 139-146. https://doi.org/10.1016/j.jtherbio.2016.09.008

Biggar, K. K., \& Storey, K. B. (2018a). Functional impact of microRNA regulation in models of extreme stress adaptation. In Journal of Molecular Cell Biology (Vol. 10, Issue 2, pp. 93-101). https://doi.org/10.1093/jmcb/mjx053

Biggar, K. K., \& Storey, K. B. (2018b). The evaluation of anoxia responsive E2F DNA binding activity in the red eared slider turtle, Trachemys scripta elegans. PeerJ, 2018(MAY). https://doi.org/10.7717/peerj.4755 
Blasco-Moreno, B., de Campos-Mata, L., Böttcher, R., García-Martínez, J., Jungfleisch, J., Nedialkova, D. D., Chattopadhyay, S., Gas, M. E., Oliva, B., Pérez-Ortín, J. E., Leidel, S. A., Choder, M., \& Díez, J. (2019). The exonuclease Xrn1 activates transcription and translation of mRNAs encoding membrane proteins. Nature Communications, 10(1). https://doi.org/10.1038/s41467-019-09199-6

Blokhina, O., Virolainen, E., \& Fagerstedt, K. V. (2003). Antioxidants, oxidative damage and oxygen deprivation stress: A review. In Annals of Botany (Vol. 91, pp. 179194). https://doi.org/10.1093/aob/mcf118

Bortolamiol-Becet, D., Hu, F., Jee, D., Wen, J., Okamura, K., Lin, C. J., Ameres, S. L., \& Lai, E. C. (2015). Selective Suppression of the Splicing-Mediated MicroRNA Pathway by the Terminal Uridyltransferase Tailor. Molecular Cell, 59(2), 217-228. https://doi.org/10.1016/j.molcel.2015.05.034

Breedon, S. A., Hadj-Moussa, H., \& Storey, K. B. (2021). Nrf2 activates antioxidant enzymes in the anoxia-tolerant red-eared slider turtle, Trachemys scripta elegans. Journal of Experimental Zoology Part A: Ecological and Integrative Physiology, 335(4), 426-435. https://doi.org/10.1002/jez.2458

Brierley, J. B. (1977). Experimental hypoxic brain damage. Journal of Clinical Pathology, S3-11, 181-187. https://doi.org/10.1136/jcp.s3-11.1.181

Brooks, S. P. J., \& Storey, K. B. (1993). De novo protein synthesis and protein phosphorylation during anoxia and recovery in the red-eared turtle. American Journal of Physiology - Regulatory Integrative and Comparative Physiology, 265(6 34-6). https://doi.org/10.1152/ajpregu.1993.265.6.r1380

Buchan, J. R., Muhlrad, D., \& Parker, R. (2008). P bodies promote stress granule assembly in Saccharomyces cerevisiae. Journal of Cell Biology, 183(3), 441-455. https://doi.org/10.1083/jcb.200807043

Buchan, J. R., \& Parker, R. (2009). Eukaryotic Stress Granules: The Ins and Outs of Translation. In Molecular Cell (Vol. 36, Issue 6, pp. 932-941). https://doi.org/10.1016/j.molcel.2009.11.020 
Bundgaard, A., James, A. M., Gruszczyk, A. V., Martin, J., Murphy, M. P., \& Fago, A. (2019). Metabolic adaptations during extreme anoxia in the turtle heart and their implications for ischemia-reperfusion injury. Scientific Reports, $9(1)$. https://doi.org/10.1038/s41598-019-39836-5

Carbon, S., Dietze, H., Lewis, S. E., Mungall, C. J., Munoz-Torres, M. C., Basu, S., Chisholm, R. L., Dodson, R. J., Fey, P., Thomas, P. D., Mi, H., Muruganujan, A., Huang, X., Poudel, S., Hu, J. C., Aleksander, S. A., McIntosh, B. K., Renfro, D. P., Siegele, D. A., ... Westerfield, M. (2017). Expansion of the gene ontology knowledgebase and resources: The gene ontology consortium. Nucleic Acids Research, 45(D1), D331-D338. https://doi.org/10.1093/nar/gkw1108

Chakravarthy, S., Sternberg, S. H., Kellenberger, C. A., \& Doudna, J. A. (2010). Substrate-specific kinetics of dicer-catalyzed RNA processing. Journal of Molecular Biology, 404(3), 392-402. https://doi.org/10.1016/j.jmb.2010.09.030

Chowdhury, A., Mukhopadhyay, J., \& Tharun, S. (2007). The decapping activator Lsm1p-7p-Pat1p complex has the intrinsic ability to distinguish between oligoadenylated and polyadenylated RNAs. Rna, 13(7), 998-1016. https://doi.org/10.1261/rna.502507

Christie, M., Boland, A., Huntzinger, E., Weichenrieder, O., \& Izaurralde, E. (2013). Structure of the PAN3 pseudokinase reveals the basis for interactions with the PAN2 deadenylase and the GW182 proteins. Molecular Cell, 51(3), 360-373. https://doi.org/10.1016/j.molcel.2013.07.011

Clark, V. M., \& Miller, A. T. (1973). Studies on anaerobic metabolism in the fresh-water turtle (Pseudemys scripta elegans). Comparative Biochemistry and Physiology -Part A: Physiology, 44(1), 55-62. https://doi.org/10.1016/0300-9629(73)90369-1

Connerly, P. L. (2010). How Do Proteins Move Through the Golgi Apparatus? Nature Education, 3(9).

Dang, Y., Kedersha, N., Low, W. K., Romo, D., Gorospe, M., Kaufman, R., Anderson, P., \& Liu, J. O. (2006). Eukaryotic initiation factor $2 \alpha$-independent pathway of 
stress granule induction by the natural product pateamine A. Journal of Biological Chemistry, 281(43), 32870-32878. https://doi.org/10.1074/jbc.M606149200

Davis, E. C., \& Jackson, D. C. (2007). Lactate uptake by skeletal bone in anoxic turtles, Trachemys scripta. Comparative Biochemistry and Physiology - A Molecular and Integrative Physiology, 146(3), 299-304. https://doi.org/10.1016/j.cbpa.2006.10.034

Decker, C. J., \& Parker, R. (2012). P-bodies and stress granules: Possible roles in the control of translation and mRNA degradation. Cold Spring Harbor Perspectives in Biology, 4(9). https://doi.org/10.1101/cshperspect.a012286

Deshmukh, M. V., Jones, B. N., Quang-Dang, D. U., Flinders, J., Floor, S. N., Kim, C., Jemielity, J., Kalek, M., Darzynkiewicz, E., \& Gross, J. D. (2008). mRNA Decapping Is Promoted by an RNA-Binding Channel in Dcp2. Molecular Cell, 29(3), 324-336. https://doi.org/10.1016/j.molcel.2007.11.027

Deshpande, O. A., \& Mohiuddin, S. S. (2020). Biochemistry, Oxidative Phophorylation. In StatPearls. http://www.ncbi.nlm.nih.gov/pubmed/31985985

Ding, L., Li, W., Li, N., Liang, L., Zhang, X., Jin, H., Shi, H., Storey, K. B., \& Hong, M. (2019). Antioxidant responses to salinity stress in an invasive species, the red-eared slider (Trachemys scripta elegans) and involvement of a TOR-Nrf2 signaling pathway. Comparative Biochemistry and Physiology Part - C: Toxicology and Pharmacology, 219, 59-67. https://doi.org/10.1016/j.cbpc.2019.02.004

Douglas, D. N., Giband, M., Altosaar, I., \& Storey, K. B. (1994). Anoxia induces changes in translatable mRNA populations in turtle organs: a possible adaptive strategy for anaerobiosis. Journal of Comparative Physiology B, 164(5), 405-414. https://doi.org/10.1007/BF00302557

Embley, T. M., \& Martin, W. (2006). Eukaryotic evolution, changes and challenges. In Nature (Vol. 440, Issue 7084, pp. 623-630). https://doi.org/10.1038/nature04546

English, S. G., Hadj-Moussa, H., \& Storey, K. B. (2018). MicroRNAs regulate survival in oxygen-deprived environments. Journal of Experimental Biology, 221(23). https://doi.org/10.1242/jeb.190579 
Eulalio, A., Behm-Ansmant, I., \& Izaurralde, E. (2007). P bodies: At the crossroads of post-transcriptional pathways. In Nature Reviews Molecular Cell Biology (Vol. 8, Issue 1, pp. 9-22). https://doi.org/10.1038/nrm2080

Eystathioy, T., Jakymiw, A., Chan, E. K. L., Séraphin, B., Cougot, N., \& Fritzler, M. J. (2003). The GW182 protein colocalizes with mRNA degradation associated proteins hDcp1 and hLSm4 in cytoplasmic GW bodies. Rna, 9(10), 1171-1173. https://doi.org/10.1261/rna.5810203

Filippov, V., Solovyev, V., Filippova, M., \& Gill, S. S. (2000). A novel type of RNase III family proteins in eukaryotes. Gene, 245(1), 213-221. https://doi.org/10.1016/S0378-1119(99)00571-5

Finnegan, E. F., \& Pasquinelli, A. E. (2013). MicroRNA biogenesis: Regulating the regulators. Critical Reviews in Biochemistry and Molecular Biology, 48(1), 51-68. https://doi.org/10.3109/10409238.2012.738643

Fraser, K. P. P., Houlihan, D. F., Lutz, P. L., Leone-Kabler, S., Manuel, L., \& Brechin, J. G. (2001). Complete suppression of protein synthesis during anoxia with no postanoxia protein synthesis debt in the red-eared slider turtle Trachemys scripta elegans. The Journal of Experimental Biology, 204(Pt 24), 4353-4360.

Fuchs, R. T., Sun, Z., Zhuang, F., \& Robb, G. B. (2015). Bias in ligation-based small RNA sequencing library construction is determined by adaptor and RNA structure. PLoS ONE, 10(5). https://doi.org/10.1371/journal.pone.0126049

Gaddi, A. V., Galuppo, P., \& Yang, J. (2017). Creatine Phosphate Administration in Cell Energy Impairment Conditions: A Summary of Past and Present Research. In Heart Lung and Circulation (Vol. 26, Issue 10, pp. 1026-1035). https://doi.org/10.1016/j.hlc.2016.12.020

Garcia-Garcia, F., Panadero, J., Dopazo, J., \& Montaner, D. (2016). Integrated gene set analysis for microRNA studies. Bioinformatics, 32(18), 2809-2816. https://doi.org/10.1093/bioinformatics/btw334

Gilks, N., Kedersha, N., Ayodele, M., Shen, L., Stoecklin, G., Dember, L. M., \& 
Anderson, P. (2004). Stress granule assembly is mediated by prion-like aggregation of TIA-1. Molecular Biology of the Cell, 15(12), 5383-5398.

https://doi.org/10.1091/mbc.E04-08-0715

Giraud-Billoud, M., Rivera-Ingraham, G. A., Moreira, D. C., Burmester, T., CastroVazquez, A., Carvajalino-Fernández, J. M., Dafre, A., Niu, C., Tremblay, N., Paital, B., Rosa, R., Storey, J. M., Vega, I. A., Zhang, W., Yepiz-Plascencia, G., ZentenoSavin, T., Storey, K. B., \& Hermes-Lima, M. (2019). Twenty years of the 'Preparation for Oxidative Stress' (POS) theory: Ecophysiological advantages and molecular strategies. In Comparative Biochemistry and Physiology -Part A :

Molecular and Integrative Physiology (Vol. 234, pp. 36-49).

https://doi.org/10.1016/j.cbpa.2019.04.004

Giwa, S., Lewis, J. K., Alvarez, L., Langer, R., Roth, A. E., Church, G. M., Markmann, J. F., Sachs, D. H., Chandraker, A., Wertheim, J. A., Rothblatt, M., Boyden, E. S., Eidbo, E., Lee, W. P. A., Pomahac, B., Brandacher, G., Weinstock, D. M., Elliott, G., Nelson, D., ... Toner, M. (2017). The promise of organ and tissue preservation to transform medicine. In Nature Biotechnology (Vol. 35, Issue 6, pp. 530-542). https://doi.org/10.1038/nbt.3889

Global Biodiversity Information Facility. (2021). Trachemys scripta subsp. elegans (Wied, 1838) in GBIF Secretariat. GBIF Backbone Taxonomy. https://doi.org/https://doi.org/10.15468/39omei

Greenway, S. C., \& Storey, K. B. (2000). Mitogen-activated protein kinases and anoxia tolerance in turtles. Journal of Experimental Zoology, 287(7), 477-484. https://doi.org/10.1002/1097-010X(20001201)287:7<477::AID-JEZ3>3.0.CO;2-4

Ha, M., \& Kim, V. N. (2014). Regulation of microRNA biogenesis. In Nature reviews. Molecular cell biology (Vol. 15, Issue 8, pp. 509-524).

Haase, A. D., Jaskiewicz, L., Zhang, H., Lainé, S., Sack, R., Gatignol, A., \& Filipowicz, W. (2005). TRBP, a regulator of cellular PKR and HIV-1 virus expression, interacts with Dicer and functions in RNA silencing. EMBO Reports, 6(10), 961-967. https://doi.org/10.1038/sj.embor.7400509 
Haddad, A., \& Mohiuddin, S. S. (2019). Biochemistry, Citric Acid Cycle. In StatPearls. http://www.ncbi.nlm.nih.gov/pubmed/31082116

Hadj-Moussa, H., Logan, S. M., Seibel, B. A., \& Storey, K. B. (2018). Potential role for microRNA in regulating hypoxia-induced metabolic suppression in jumbo squids. Biochimica et Biophysica Acta - Gene Regulatory Mechanisms. https://doi.org/10.1016/j.bbagrm.2018.04.007

Hadj-Moussa, H., Pamenter, M. E., \& Storey, K. B. (2021). Hypoxic naked mole-rat brains use microRNA to coordinate hypometabolic fuels and neuroprotective defenses. Journal of Cellular Physiology, 236(7), 5080-5097. https://doi.org/10.1002/jcp.30216

Hadj-Moussa, H., \& Storey, K. B. (2018). Micromanaging freeze tolerance: the biogenesis and regulation of neuroprotective microRNAs in frozen brains. Cellular and Molecular Life Sciences, 75(19), 3635-3647. https://doi.org/10.1007/s00018018-2821-0

Hadj-Moussa, H., \& Storey, K. B. (2019). Bringing nature back: using hibernation to reboot organ preservation. FEBS Journal, 286(6), 1094-1100. https://doi.org/10.1111/febs.14683

Hadj-Moussa, H., \& Storey, K. B. (2020). The OxymiR response to oxygen limitation: A comparative microRNA perspective. In Journal of Experimental Biology (Vol. 223, Issue 10). https://doi.org/10.1242/jeb.204594

Hadj-Moussa, H., Zhang, J., Pifferi, F., Perret, M., \& Storey, K. B. (2020). Profiling torpor-responsive microRNAs in muscles of the hibernating primate Microcebus murinus. Biochimica et Biophysica Acta - Gene Regulatory Mechanisms, 1863(1). https://doi.org/10.1016/j.bbagrm.2019.194473

Hammond, S. M. (2015). An overview of microRNAs. In Advanced Drug Delivery Reviews. https://doi.org/10.1016/j.addr.2015.05.001

Han, J., Pedersen, J. S., Kwon, S. C., Belair, C. D., Kim, Y. K., Yeom, K. H., Yang, W. Y., Haussler, D., Blelloch, R., \& Kim, V. N. (2009). Posttranscriptional 
Crossregulation between Drosha and DGCR8. Cell, 136(1), 75-84.

https://doi.org/10.1016/j.cell.2008.10.053

Hanahan, D., \& Weinberg, R. A. (2011). Hallmarks of cancer: The next generation. In Cell (Vol. 144, Issue 5, pp. 646-674). https://doi.org/10.1016/j.cell.2011.02.013

Hawkins, L. J., \& Storey, K. B. (2020). MicroRNA expression in the heart of Xenopus laevis facilitates metabolic adaptation to dehydration. Genomics, 112(5), 35253536. https://doi.org/10.1016/j.ygeno.2020.04.003

He, L., \& Hannon, G. J. (2004). MicroRNAs: Small RNAs with a big role in gene regulation. In Nature Reviews Genetics (Vol. 5, Issue 7, pp. 522-531). https://doi.org/10.1038/nrg1379

Hegde, A. N. (2010). Ubiquitin-dependent protein degradation. In Comprehensive Natural Products II: Chemistry and Biology (Vol. 5, pp. 699-752). https://doi.org/10.1016/b978-008045382-8.00697-3

Herbert, C. V., \& Jackson, D. C. (1985). Temperature Effects on the Responses to Prolonged Submergence in the Turtle Chrysemys picta bellii. II. Metabolic Rate, Blood Acid-Base and Ionic Changes, and Cardiovascular Function in Aerated and Anoxic Water. Physiological Zoology, 58(6), 670-681. https://doi.org/10.1086/physzool.58.6.30156071

Hermes-Lima, M., \& Zenteno-Savín, T. (2002). Animal response to drastic changes in oxygen availability and physiological oxidative stress. Comparative Biochemistry and Physiology - C Toxicology and Pharmacology, 133(4), 537-556. https://doi.org/10.1016/S1532-0456(02)00080-7

Hochachka, P. W. (1988). Metabolic suppression and oxygen availability. Canadian Journal of Zoology, 66(1), 152-158. https://doi.org/10.1139/z88-021

Hochachka, P. W., Buck, L. T., Doll, C. J., \& Land, S. C. (1996). Unifying theory of hypoxia tolerance: molecular/metabolic defense and rescue mechanisms for surviving oxygen lack. Proceedings of the National Academy of Sciences of the United States of America, 93(18), 9493-9498. 
Hochachka, P. W., \& Lutz, P. L. (2001). Mechanism, origin, and evolution of anoxia tolerance in animals. Comparative Biochemistry and Physiology - B Biochemistry and Molecular Biology, 130(4), 435-459. https://doi.org/10.1016/S10964959(01)00408-0

Horman, S. R., Janas, M. M., Litterst, C., Wang, B., MacRae, I. J., Sever, M. J., Morrissey, D. V., Graves, P., Luo, B., Umesalma, S., Qi, H. H., Miraglia, L. J., Novina, C. D., \& Orth, A. P. (2013). Akt-mediated phosphorylation of argonaute 2 downregulates cleavage and upregulates translational repression of MicroRNA targets. Molecular Cell, 50(3), 356-367. https://doi.org/10.1016/j.molcel.2013.03.015

Hoyeck, M. P., Hadj-Moussa, H., \& Storey, K. B. (2019). Estivation-responsive microRNAs in a hypometabolic terrestrial snail. PeerJ, 2019(2). https://doi.org/10.7717/peerj.6515

Hoyle, N. P., Castelli, L. M., Campbell, S. G., Holmes, L. E. A., \& Ashe, M. P. (2007). Stress-dependent relocalization of translationally primed mRNPs to cytoplasmic granules that are kinetically and spatially distinct from P-bodies. Journal of Cell Biology, 179(1), 65-74. https://doi.org/10.1083/jcb.200707010

Hubstenberger, A., Courel, M., Bénard, M., Souquere, S., Ernoult-Lange, M., Chouaib, R., Yi, Z., Morlot, J. B., Munier, A., Fradet, M., Daunesse, M., Bertrand, E., Pierron, G., Mozziconacci, J., Kress, M., \& Weil, D. (2017). P-Body Purification Reveals the Condensation of Repressed mRNA Regulons. Molecular Cell, 68(1), 144-157.e5. https://doi.org/10.1016/j.molcel.2017.09.003

Huntzinger, E., \& Izaurralde, E. (2011). Gene silencing by microRNAs: Contributions of translational repression and mRNA decay. In Nature Reviews Genetics (Vol. 12, Issue 2, pp. 99-110). https://doi.org/10.1038/nrg2936

Jackson, D. C. (1968). Metabolic depression and oxygen depletion in the diving turtle. Journal of Applied Physiology, 24(4), 503-509. https://doi.org/10.1152/jappl.1968.24.4.503 
Jackson, D. C. (2000). How a turtle's shell helps it survive prolonged anoxic acidosis. News in Physiological Sciences, 15(4), 181-185. https://doi.org/10.1152/physiologyonline.2000.15.4.181

Jackson, D. C. (2002). Hibernating without oxygen: Physiological adaptations of the painted turtle. In Journal of Physiology (Vol. 543, Issue 3, pp. 731-737). https://doi.org/10.1113/jphysiol.2002.024729

Jackson, D. C., Crocker, C. E., \& Ultsch, G. R. (2000). Bone and shell contribution to lactic acid buffering of submerged turtles Chrysemys picta bellii at $3^{\circ} \mathrm{C}$. American Journal of Physiology - Regulatory Integrative and Comparative Physiology, 278(6 47-6). https://doi.org/10.1152/ajpregu.2000.278.6.r1564

Jackson, D. C., Ramsey, A. L., Paulson, J. M., Crocker, C. E., \& Ultsch, G. R. (2000). Lactic acid buffering by bone and shell in anoxic softshell and painted turtles. In Physiological and Biochemical Zoology (Vol. 73, Issue 3, pp. 290-297). https://doi.org/10.1086/316754

Jackson, D. C., Rauer, E. M., Feldman, R. A., \& Reese, S. A. (2004). Avenues of extrapulmonary oxygen uptake in western painted turtles (Chrysemys picta belli) at $10^{\circ} \mathrm{C}$. Comparative Biochemistry and Physiology - A Molecular and Integrative Physiology, 139(2), 221-227. https://doi.org/10.1016/j.cbpb.2004.09.005

Jackson, D. C., \& Ultsch, G. R. (2010). Physiology of hibernation under the ice by turtles and frogs. Journal of Experimental Zoology Part A: Ecological Genetics and Physiology, 313 A(6), 311-327. https://doi.org/10.1002/jez.603

Jaenisch, R., \& Bird, A. (2003). Epigenetic regulation of gene expression: How the genome integrates intrinsic and environmental signals. In Nature Genetics (Vol. 33, Issue 3S, pp. 245-254). https://doi.org/10.1038/ng1089

Jain, S., \& Parker, R. (2013). The discovery and analysis of P bodies. Advances in Experimental Medicine and Biology, 768, 23-43. https://doi.org/10.1007/978-14614-5107-5_3

Jakymiw, A., Lian, S., Eystathioy, T., Li, S., Satoh, M., Hamel, J. C., Fritzler, M. J., \& 
Chan, E. K. L. (2005). Disruption of GW bodies impairs mammalian RNA interference. Nature Cell Biology, 7(12), 1167-1174.

https://doi.org/10.1038/ncb1334

Jakymiw, A., Pauley, K. M., Li, S., Ikeda, K., Lian, S., Eystathioy, T., Satoh, M., Fritzler, M. J., \& Chan, E. K. L. (2007). The role of GW/P-bodies in RNA processing and silencing. Journal of Cell Science, 120(8), 1317-1323.

https://doi.org/10.1242/jcs.03429

Jonas, S., \& Izaurralde, E. (2015). Towards a molecular understanding of microRNAmediated gene silencing. In Nature Reviews Genetics (Vol. 16, Issue 7, pp. 421433). https://doi.org/10.1038/nrg3965

Kadener, S., Rodriguez, J., Abruzzi, K. C., Khodor, Y. L., Sugino, K., Marr, M. T., Nelson, S., \& Rosbash, M. (2009). Genome-wide identification of targets of the drosha-pasha/DGCR8 complex. Rna, 15(4), 537-545.

https://doi.org/10.1261/rna.1319309

Kalvari, I., Argasinska, J., Quinones-Olvera, N., Nawrocki, E. P., Rivas, E., Eddy, S. R., Bateman, A., Finn, R. D., \& Petrov, A. I. (2018). Rfam 13.0: Shifting to a genomecentric resource for non-coding RNA families. Nucleic Acids Research, 46(D1), D335-D342. https://doi.org/10.1093/nar/gkx1038

Kanehisa, M., \& Goto, S. (2000). KEGG: Kyoto Encyclopedia of Genes and Genomes. In Nucleic Acids Research (Vol. 28, Issue 1, pp. 27-30). https://doi.org/10.1093/nar/28.1.27

Kang, N. N., \& Koo, J. H. (2012). Olfactory receptors in non-chemosensory tissues. In BMB Reports (Vol. 45, Issue 11, pp. 612-622). https://doi.org/10.5483/BMBRep.2012.45.11.232

Kedersha, N., \& Anderson, P. (2007). Mammalian Stress Granules and Processing Bodies. In Methods in Enzymology (Vol. 431, pp. 61-81). https://doi.org/10.1016/S0076-6879(07)31005-7

Kedersha, N., Gupta, M., Li, W., Miller, I., \& Anderson, P. (1999). RNA-binding 
proteins TIA-1 and TIAR link the phosphorylation of eIF-2 $\alpha$ to the assembly of mammalian stress granules. Journal of Cell Biology, 147(7), 1431-1441. https://doi.org/10.1083/jcb.147.7.1431

Kedersha, N., Stoecklin, G., Ayodele, M., Yacono, P., Lykke-Andersen, J., Fitzler, M. J., Scheuner, D., Kaufman, R. J., Golan, D. E., \& Anderson, P. (2005). Stress granules and processing bodies are dynamically linked sites of mRNP remodeling. Journal of Cell Biology, 169(6), 871-884. https://doi.org/10.1083/jcb.200502088

Kesaraju, S., Nayak, G., Prentice, H. M., \& Milton, S. L. (2014). Upregulation of Hsp72 mediates anoxia/reoxygenation neuroprotection in the freshwater turtle via modulation of ROS. Brain Research, 1582, 247-256. https://doi.org/10.1016/j.brainres.2014.07.044

Kesaraju, S., Schmidt-Kastner, R., Prentice, H. M., \& Milton, S. L. (2009). Modulation of stress proteins and apoptotic regulators in the anoxia tolerant turtle brain. Journal of Neurochemistry, 109(5), 1413-1426. https://doi.org/10.1111/j.14714159.2009.06068.x

Khvorova, A., Reynolds, A., \& Jayasena, S. D. (2003). Functional siRNAs and miRNAs exhibit strand bias. Cell, 115(2), 209-216. https://doi.org/10.1016/S00928674(03)00801-8

Kim, V. N. (2005). MicroRNA biogenesis: Coordinated cropping and dicing. Nature Reviews Molecular Cell Biology, 6(5), 376-385. https://doi.org/10.1038/nrm1644

Koscianska, E., Starega-Roslan, J., \& Krzyzosiak, W. J. (2011). The role of dicer protein partners in the processing of microRNA precursors. PLoS ONE, 6(12). https://doi.org/10.1371/journal.pone.0028548

Krivoruchko, A., \& Storey, K. B. (2010a). Epigenetics in anoxia tolerance: A role for histone deacetylases. Molecular and Cellular Biochemistry, 342(1-2), 151-161. https://doi.org/10.1007/s11010-010-0479-5

Krivoruchko, A., \& Storey, K. B. (2010b). Forever young: Mechanisms of natural anoxia tolerance and potential links to longevity. In Oxidative Medicine and Cellular 
Longevity (Vol. 3, Issue 3, pp. 186-198). https://doi.org/10.4161/oxim.3.3.12356

Krivoruchko, A., \& Storey, K. B. (2010c). Molecular mechanisms of turtle anoxia tolerance: A role for NF-кB. Gene, 450(1-2), 63-69. https://doi.org/10.1016/j.gene.2009.10.005

Krivoruchko, A., \& Storey, K. B. (2010d). Regulation of the heat shock response under anoxia in the turtle, Trachemys scripta elegans. Journal of Comparative Physiology. B, Biochemical, Systemic, and Environmental Physiology, 180(3), 403-414. https://doi.org/10.1007/s00360-009-0414-9

Krivoruchko, A., \& Storey, K. B. (2013). Activation of the unfolded protein response during anoxia exposure in the turtle Trachemys scripta elegans. Molecular and Cellular Biochemistry, 374(1-2), 91-103. https://doi.org/10.1007/s11010-012-15083

Krivoruchko, A., \& Storey, K. B. (2015). Turtle anoxia tolerance: Biochemistry and gene regulation. In Biochimica et Biophysica Acta - General Subjects (Vol. 1850, Issue 6, pp. 1188-1196). https://doi.org/10.1016/j.bbagen.2015.02.001

Kurisu, K., \& Yenari, M. A. (2018). Therapeutic hypothermia for ischemic stroke; pathophysiology and future promise. In Neuropharmacology (Vol. 134, pp. 302309). https://doi.org/10.1016/j.neuropharm.2017.08.025

Kurtz, R., Steinberg, L. G., Betcher, M., Fowler, D., \& Shepard, B. D. (2020). The Sensing Liver: Localization and Ligands for Hepatic Murine Olfactory and Taste Receptors. Frontiers in Physiology, 11. https://doi.org/10.3389/fphys.2020.574082

Lakin, N. D., \& Jackson, S. P. (1999). Regulation of p53 in response to DNA damage. In Oncogene (Vol. 18, Issue 53, pp. 7644-7655). https://doi.org/10.1038/sj.onc. 1203015

Land, S. C., Buck, L. T., \& Hochachka, P. W. (1993). Response of protein synthesis to anoxia and recovery in anoxia-tolerant hepatocytes. American Journal of Physiology - Regulatory Integrative and Comparative Physiology, 265(1 34-1). https://doi.org/10.1152/ajpregu.1993.265.1.r41 
Langmead, B., Trapnell, C., Pop, M., \& Salzberg, S. L. (2009). Ultrafast and memoryefficient alignment of short DNA sequences to the human genome. Genome Biology, 10(3). https://doi.org/10.1186/gb-2009-10-3-r25

Larson, J., Drew, K. L., Folkow, L. P., Milton, S. L., \& Park, T. J. (2014). No oxygen? No problem! intrinsic brain tolerance to hypoxia in vertebrates. In Journal of Experimental Biology (Vol. 217, Issue 7, pp. 1024-1039). https://doi.org/10.1242/jeb.085381

Law, C. W., Chen, Y., Shi, W., \& Smyth, G. K. (2014). Voom: Precision weights unlock linear model analysis tools for RNA-seq read counts. Genome Biology, 15(2). https://doi.org/10.1186/gb-2014-15-2-r29

Lee, R. C., Feinbaum, R. L., \& Ambros, V. (1993). The C. elegans heterochronic gene lin-4 encodes small RNAs with antisense complementarity to lin-14. Cell, 75(5), 843-854. https://doi.org/10.1016/0092-8674(93)90529-Y

Lee, Y., Hur, I., Park, S.-Y. Y., Kim, Y.-K. K., Suh, M. R., Kim, V. N., Mi, R. S., \& Kim, V. N. (2006). The role of PACT in the RNA silencing pathway. EMBO Journal, 25(3), 522-532. https://doi.org/10.1038/sj.emboj.7600942

Lee, Y., Jeon, K., Lee, J. T., Kim, S., \& Kim, V. N. (2002). MicroRNA maturation: Stepwise processing and subcellular localization. EMBO Journal, 21(17), 46634670. https://doi.org/10.1093/emboj/cdf476

Lei, E. P., \& Silver, P. A. (2002). Protein and RNA export from the nucleus. In Developmental Cell (Vol. 2, Issue 3, pp. 261-272). https://doi.org/10.1016/S15345807(02)00134-X

Lenz, M., Muller, F. J., Zenke, M., \& Schuppert, A. (2016). Principal components analysis and the reported low intrinsic dimensionality of gene expression microarray data. Scientific Reports, 6. https://doi.org/10.1038/srep25696

Leung, A. K. L., \& Sharp, P. A. (2010). MicroRNA functions in stress responses. Molecular Cell, 40(2), 205-215.

Leung, A. K. L., \& Sharp, P. A. (2006). Function and localization of microRNAs in 
mammalian cells. Cold Spring Harbor Symposia on Quantitative Biology, 71, 2938. https://doi.org/10.1101/sqb.2006.71.049

Li, H., Handsaker, B., Wysoker, A., Fennell, T., Ruan, J., Homer, N., Marth, G., Abecasis, G., \& Durbin, R. (2009). The Sequence Alignment/Map format and SAMtools. Bioinformatics, 25(16), 2078-2079. https://doi.org/10.1093/bioinformatics/btp352

Li, Y. R., King, O. D., Shorter, J., \& Gitler, A. D. (2013). Stress granules as crucibles of ALS pathogenesis. In Journal of Cell Biology (Vol. 201, Issue 3, pp. 361-372). https://doi.org/10.1083/jcb.201302044

Liu, J., Rivas, F. V., Wohlschlegel, J., Yates, J. R., Parker, R., \& Hannon, G. J. (2005). A role for the P-body component GW182 in microRNA function. Nature Cell Biology, 7(12), 1161-1166. https://doi.org/10.1038/ncb1333

Liu, J., Valencia-Sanchez, M. A., Hannon, G. J., \& Parker, R. (2005). MicroRNAdependent localization of targeted mRNAs to mammalian P-bodies. Nature Cell Biology, 7(7), 719-723. https://doi.org/10.1038/ncb1274

Liu, N., \& Pan, T. (2015). RNA epigenetics. In Translational Research (Vol. 165, Issue 1, pp. 28-35). https://doi.org/10.1016/j.trsl.2014.04.003

Lobo, V., Patil, A., Phatak, A., \& Chandra, N. (2010). Free radicals, antioxidants and functional foods: Impact on human health. In Pharmacognosy Reviews (Vol. 4, Issue 8, pp. 118-126). https://doi.org/10.4103/0973-7847.70902

Logan, S. M., \& Storey, K. B. (2021). MicroRNA expression patterns in the brown fat of hibernating 13-lined ground squirrels. Genomics, 113(2), 769-781. https://doi.org/10.1016/j.ygeno.2021.01.017

Longeville, S., \& Stingaciu, L. R. (2017). Hemoglobin diffusion and the dynamics of oxygen capture by red blood cells. Scientific Reports, 7(1). https://doi.org/10.1038/s41598-017-09146-9

Luu, B. E., Lefai, E., Giroud, S., Swenson, J. E., Chazarin, B., Gauquelin-Koch, G., Arnemo, J. M., Evans, A. L., Bertile, F., \& Storey, K. B. (2020). MicroRNAs 
facilitate skeletal muscle maintenance and metabolic suppression in hibernating brown bears. Journal of Cellular Physiology, 235(4), 3984-3993. https://doi.org/10.1002/jcp.29294

Lyons, P. J., Govaere, L., Crapoulet, N., Storey, K. B., \& Morin, P. J. (2016). Characterization of cold-associated microRNAs in the freeze-tolerant gall fly Eurosta solidaginis using high-throughput sequencing. Comparative Biochemistry and Physiology - Part D: Genomics and Proteomics, 20, 95-100. https://doi.org/10.1016/j.cbd.2016.08.007

Ma, E., MacRae, I. J., Kirsch, J. F., \& Doudna, J. A. (2008). Autoinhibition of Human Dicer by Its Internal Helicase Domain. Journal of Molecular Biology, 380(1), 237 243. https://doi.org/10.1016/j.jmb.2008.05.005

MacIas, S., Plass, M., Stajuda, A., Michlewski, G., Eyras, E., \& Cáceres, J. F. (2012). DGCR8 HITS-CLIP reveals novel functions for the Microprocessor. Nature Structural and Molecular Biology, 19(8), 760-766. https://doi.org/10.1038/nsmb.2344

MacRae, I. J., \& Doudna, J. A. (2007). Ribonuclease revisited: structural insights into ribonuclease III family enzymes. In Current Opinion in Structural Biology (Vol. 17, Issue 1, pp. 138-145). https://doi.org/10.1016/j.sbi.2006.12.002

Martin, M. (2011). Cutadapt removes adapter sequences from high-throughput sequencing reads. EMBnet.Journal, 17(1), 10. https://doi.org/10.14806/ej.17.1.200

Maßberg, D., \& Hatt, H. (2018). Human olfactory receptors: Novel cellular functions outside of the nose. In Physiological Reviews (Vol. 98, Issue 3, pp. 1739-1763). https://doi.org/10.1152/PHYSREV.00013.2017

Mattice, A. M. S., MacLean, I. A., Childers, C. L., \& Storey, K. B. (2018). Characterization of pyruvate kinase from the anoxia tolerant turtle, Trachemys scripta elegans: A potential role for enzyme methylation during metabolic rate depression. PeerJ, 2018(6). https://doi.org/10.7717/peerj.4918

Mazroui, R., Sukarieh, R., Bordeleau, M. E., Kaufman, R. J., Northcote, P., Tanaka, J., 
Gallouzi, I., \& Pelletier, J. (2006). Inhibition of ribosome recruitment induces stress granule formation independently of eukaryotic initiation factor $2 \alpha$ phosphorylation. Molecular Biology of the Cell, 17(10), 4212-4219. https://doi.org/10.1091/mbc.E0604-0318

Mehrani, H., \& Storey, K. B. (1995). cAMP-dependent protein kinase and anoxia survival in turtles: Purification and properties of liver PKA. Molecular and Cellular Biochemistry, 145(1), 81-88. https://doi.org/10.1007/BF00925717

Mehrani, H., \& Storey, K. B. (1996). Liver protein kinase C isozymes: Properties and enzyme role in a vertebrate facultative anaerobe. International Journal of Biochemistry and Cell Biology, 28(11), 1257-1269. https://doi.org/10.1016/S13572725(96)00062-3

Melkonian, E. A., \& Schury, M. P. (2019). Biochemistry, Anaerobic Glycolysis. In StatPearls. http://www.ncbi.nlm.nih.gov/pubmed/31536301

Milton, S. L., Nayak, G., Kesaraju, S., Kara, L., \& Prentice, H. M. (2007). Suppression of reactive oxygen species production enhances neuronal survival in vitro and in vivo in the anoxia-tolerant turtle Trachemys scripta. Journal of Neurochemistry, 101(4), 993-1001. https://doi.org/10.1111/j.1471-4159.2007.04466.x

Mollet, S., Cougot, N., Wilczynska, A., Dautry, F., Kress, M., Bertrand, E., \& Weil, D. (2008). Translationally repressed mRNA transiently cycles through stress granules during stress. Molecular Biology of the Cell, 19(10), 4469-4479. https://doi.org/10.1091/mbc.E08-05-0499

Montano, M. (2011). MicroRNAs: MiRRORS of health and disease. In Translational Research (Vol. 157, Issue 4, pp. 157-162). https://doi.org/10.1016/j.trsl.2011.02.001

Morin, P., \& Storey, K. B. (2005). Cloning and expression of hypoxia-inducible factor $1 \alpha$ from the hibernating ground squirrel, Spermophilus tridecemlineatus. Biochimica et Biophysica Acta - Gene Structure and Expression, 1729(1), 32-40. https://doi.org/10.1016/j.bbaexp.2005.02.009

Morin, P., \& Storey, K. B. (2009). Mammalian hibernation: Differential gene expression 
and novel application of epigenetic controls. In International Journal of Developmental Biology (Vol. 53, Issues 2-3, pp. 433-442).

https://doi.org/10.1387/ijdb.082643pm

Nishihara, T., Zekri, L., Braun, J. E., \& Izaurralde, E. (2013). MiRISC recruits decapping factors to miRNA targets to enhance their degradation. Nucleic Acids Research, 4l(18), 8692-8705. https://doi.org/10.1093/nar/gkt619

O’Brien, J., Hayder, H., Zayed, Y., \& Peng, C. (2018). Overview of microRNA biogenesis, mechanisms of actions, and circulation. In Frontiers in Endocrinology (Vol. 9, Issue AUG). https://doi.org/10.3389/fendo.2018.00402

Okamura, K., Phillips, M. D., Tyler, D. M., Duan, H., Chou, Y. T., \& Lai, E. C. (2008). The regulatory activity of microRNA* species has substantial influence on microRNA and 3' UTR evolution. Nature Structural and Molecular Biology, 15(4), 354-363. https://doi.org/10.1038/nsmb.1409

Paciello, G., Acquaviva, A., Ficarra, E., Deriu, M. A., \& MacIi, E. (2011). A molecular dynamics study of a miRNA:mRNA interaction. Journal of Molecular Modeling, 17(11), 2895-2906. https://doi.org/10.1007/s00894-011-0991-x

Parker, R. (2012). RNA degradation in Saccharomyces cerevisae. Genetics, 191(3), 671702. https://doi.org/10.1534/genetics.111.137265

Parker, R., \& Sheth, U. (2007). P Bodies and the Control of mRNA Translation and Degradation. In Molecular Cell (Vol. 25, Issue 5, pp. 635-646). https://doi.org/10.1016/j.molcel.2007.02.011

Patel, S., \& Mohiuddin, S. S. (2020). Physiology, Oxygen Transport And Carbon Dioxide Dissociation Curve. In StatPearls. http://www.ncbi.nlm.nih.gov/pubmed/30969637

Pittman, R. N. (2016). Regulation of Tissue Oxygenation Second Edition. Colloquium Series on Integrated Systems Physiology: From Molecule to Function, 8(2), i-99. https://doi.org/10.4199/c00140ed2v01y201606isp065

Popel, A. S. (1989). Theory of oxygen transport to tissue. Critical Reviews in Biomedical Engineering, 17(3), 257-321. 
Prentice, H. M., Milton, S. L., Scheurle, D., \& Lutz, P. L. (2004). The upregulation of cognate and inducible heat shock proteins in the anoxic turtle brain. Journal of Cerebral Blood Flow and Metabolism, 24(7), 826-828. https://doi.org/10.1097/01.WCB.0000126565.27130.79

R Core Team. (2017). R: A language and environment for statistical computing. https://www.r-project.org/

Raabe, C. A., Tang, T. H., Brosius, J., \& Rozhdestvensky, T. S. (2014). Biases in small RNA deep sequencing data. Nucleic Acids Research, 42(3), 1414-1426. https://doi.org/10.1093/nar/gkt1021

Reichholf, B., Herzog, V. A., Fasching, N., Manzenreither, R. A., Sowemimo, I., \& Ameres, S. L. (2019). Time-Resolved Small RNA Sequencing Unravels the Molecular Principles of MicroRNA Homeostasis. Molecular Cell, 75(4), 756768.e7. https://doi.org/10.1016/j.molcel.2019.06.018

Reimão-Pinto, M. M., Ignatova, V., Burkard, T. R., Hung, J. H., Manzenreither, R. A., Sowemimo, I., Herzog, V. A., Reichholf, B., Fariña-Lopez, S., \& Ameres, S. L. (2015). Uridylation of RNA Hairpins by Tailor Confines the Emergence of MicroRNAs in Drosophila. Molecular Cell, 59(2), 203-216. https://doi.org/10.1016/j.molcel.2015.05.033

Reinhart, B. J., Slack, F. J., Basson, M., Pasquienell1, A. E., Bettlnger, J. C., Rougvle, A. E., Horvitz, H. R., \& Ruvkun, G. (2000). The 21-nucleotide let-7 RNA regulates developmental timing in Caenorhabditis elegans. Nature. https://doi.org/10.1038/35002607

Rice, M. E., Lee, E. J. K., \& Choy, Y. (1995). High Levels of Ascorbic Acid, Not Glutathione, in the CNS of Anoxia-Tolerant Reptiles Contrasted with Levels in Anoxia-Intolerant Species. Journal of Neurochemistry, 64(4), 1790-1799. https://doi.org/10.1046/j.1471-4159.1995.64041790.x

Ritchie, M. E., Phipson, B., Wu, D., Hu, Y., Law, C. W., Shi, W., \& Smyth, G. K. (2015). Limma powers differential expression analyses for RNA-sequencing and 
microarray studies. Nucleic Acids Research, 43(7), e47.

https://doi.org/10.1093/nar/gkv007

Ross Buchan, J. (2014). MRNP granules Assembly, function, and connections with disease. In RNA Biology (Vol. 11, Issue 8, pp. 1019-1030). https://doi.org/10.4161/15476286.2014.972208

Ruby, J. G., Jan, C. H., \& Bartel, D. P. (2007). Intronic microRNA precursors that bypass Drosha processing. Nature, 448(7149), 83-86. https://doi.org/10.1038/nature05983

Sai lakshmi, S., \& Agrawal, S. (2008). piRNABank: A web resource on classified and clustered Piwi-interacting RNAs. Nucleic Acids Research, 36(SUPPL. 1). https://doi.org/10.1093/nar/gkm696

Salehi, S., Tran, K., \& Grayson, W. L. (2018). Advances in perfusion systems for solid organ preservation. In Yale Journal of Biology and Medicine (Vol. 91, Issue 3, pp. 301-312).

Schenone, A. L., Cohen, A., Patarroyo, G., Harper, L., Wang, X. F., Shishehbor, M. H., Menon, V., \& Duggal, A. (2016). Therapeutic hypothermia after cardiac arrest: A systematic review/meta-analysis exploring the impact of expanded criteria and targeted temperature. In Resuscitation (Vol. 108, pp. 102-110). https://doi.org/10.1016/j.resuscitation.2016.07.238

Schirrmeister, B. E., Gugger, M., \& Donoghue, P. C. J. (2015). Cyanobacteria and the Great Oxidation Event: Evidence from genes and fossils. Palaeontology, 58(5), 769-785. https://doi.org/10.1111/pala.12178

Schwartz, D. C., \& Parker, R. (2000). mRNA Decapping in Yeast Requires Dissociation of the Cap Binding Protein, Eukaryotic Translation Initiation Factor 4E. Molecular and Cellular Biology, 20(21), 7933-7942. https://doi.org/10.1128/mcb.20.21.79337942.2000

Semenza, G. L. (2007). Hypoxia-inducible factor 1 (HIF-1) pathway. In Science's STKE : signal transduction knowledge environment (Vol. 2007, Issue 407). https://doi.org/10.1126/stke.4072007cm8 
Shen, J., Xia, W., Khotskaya, Y. B., Huo, L., Nakanishi, K., Lim, S. O., Du, Y., Wang, Y., Chang, W. C., Chen, C. H., Hsu, J. L., Wu, Y., Lam, Y. C., James, B. P., Liu, X., Liu, C. G., Patel, D. J., \& Hung, M. C. (2013). EGFR modulates microRNA maturation in response to hypoxia through phosphorylation of AGO2. Nature, 497(7449), 383-387. https://doi.org/10.1038/nature12080

Spriggs, K. A., Bushell, M., \& Willis, A. E. (2010). Translational Regulation of Gene Expression during Conditions of Cell Stress. In Molecular Cell (Vol. 40, Issue 2, pp. 228-237). https://doi.org/10.1016/j.molcel.2010.09.028

Stancic, A., Jankovic, A., Korac, A., Cirovic, D., Otasevic, V., Storey, K. B., \& Korac, B. (2018). A lesson from the oxidative metabolism of hibernator heart: Possible strategy for cardioprotection. Comparative Biochemistry and Physiology Part - B:

Biochemistry and Molecular Biology, 219-220, 1-9. https://doi.org/10.1016/j.cbpb.2018.02.004

Standart, N., \& Weil, D. (2018). P-Bodies: Cytosolic Droplets for Coordinated mRNA Storage. In Trends in Genetics (Vol. 34, Issue 8, pp. 612-626). https://doi.org/10.1016/j.tig.2018.05.005

Stecyk, J. A. W., Couturier, C. S., Fagernes, C. E., Ellefsen, S., \& Nilsson, G. E. (2012). Quantification of heat shock protein mRNA expression in warm and cold anoxic turtles (Trachemys scripta) using an external RNA control for normalization. Comparative Biochemistry and Physiology - Part D: Genomics and Proteomics, 7(1), 59-72. https://doi.org/10.1016/j.cbd.2011.11.001

Storey, K. B. (1996). Oxidative stress: Animal adaptations in nature. Brazilian Journal of Medical and Biological Research, 29(12), 1715-1733.

Storey, K. B. (2004). Adventures in oxygen metabolism. In Comparative Biochemistry and Physiology - B Biochemistry and Molecular Biology (Vol. 139, Issue 3 SPEC.ISS., pp. 359-369). https://doi.org/10.1016/j.cbpc.2004.02.018

Storey, K. B. (2007). Anoxia tolerance in turtles: Metabolic regulation and gene expression. In Comparative Biochemistry and Physiology - A Molecular and 
Integrative Physiology (Vol. 147, Issue 2 SPEC. ISS., pp. 263-276).

https://doi.org/10.1016/j.cbpa.2006.03.019

Storey, K. B. (2015). Regulation of hypometabolism: Insights into epigenetic controls. In Journal of Experimental Biology (Vol. 218, Issue 1, pp. 150-159). https://doi.org/10.1242/jeb.106369

Storey, K. B., \& Storey, J. M. (1990). Metabolic rate depression and biochemical adaptation in anaerobiosis, hibernation and estivation. Quarterly Review of Biology, 65(2), 145-174. https://doi.org/10.1086/416717

Storey, K. B., \& Storey, J. M. (2004). Metabolic rate depression in animals: Transcriptional and translational controls. In Biological Reviews of the Cambridge Philosophical Society (Vol. 79, Issue 1, pp. 207-233). https://doi.org/10.1017/S1464793103006195

Sweet, T. J., Boyer, B., Hu, W., Baker, K. E., \& Coller, J. (2007). Microtubule disruption stimulates P-body formation. Rna, 13(4), 493-502. https://doi.org/10.1261/rna.355807

Swisher, K. D., \& Parker, R. (2010). Localization to, and effects of Pbp1, Pbp4, Lsm12, Dhh1, and Pab1 on stress granules in Saccharomyces cerevisiae. PLoS ONE, 5(4). https://doi.org/10.1371/journal.pone.0010006

Szereszewski, K. E., \& Storey, K. B. (2018). Translational regulation in the anoxic turtle, Trachemys scripta elegans. Molecular and Cellular Biochemistry, 445(1-2), 13-23. https://doi.org/10.1007/s11010-017-3247-y

Teixeira, D., Sheth, U., Valencia-Sanchez, M. A., Brengues, M., \& Parker, R. (2005). Processing bodies require RNA for assembly and contain nontranslating mRNAs. Rna, 11(4), 371-382. https://doi.org/10.1261/rna.7258505

Tessier, S. N., Audas, T. E., Wu, C. W., Lee, S., \& Storey, K. B. (2014). The involvement of mRNA processing factors TIA-1, TIAR, and PABP-1 during mammalian hibernation. Cell Stress and Chaperones, 19(6). https://doi.org/10.1007/s12192-014-0505-8 
Tourrière, H., Chebli, K., Zekri, L., Courselaud, B., Blanchard, J. M., Bertrand, E., \& Tazi, J. (2003). The RasGAP-associated endoribonuclease G3BP assembles stress granules. Journal of Cell Biology, 160(6), 823-831.

https://doi.org/10.1083/jcb.200212128

Tutar, L., \& Tutar, Y. (2010). Heat Shock Proteins; An Overview. Current Pharmaceutical Biotechnology, 11(2), 216-222. https://doi.org/10.2174/138920110790909632

Ultsch, G. R. (1985). The viability of nearctic freshwater turtles submerged in anoxia and normoxia at 3 and $10^{\circ} \mathrm{C}$. Comparative Biochemistry and Physiology -- Part A: Physiology, 81(3), 607-611. https://doi.org/10.1016/0300-9629(85)91035-7

Ultsch, G. R. (1989). Ecology and physiology of hibernation and overwintering among freshwater fishes, turtles and snakes. Biological Reviews - Cambridge Philosophical Society, 64(4), 435-516. https://doi.org/10.1111/j.1469-185x.1989.tb00683.x

Ultsch, G. R. (2006). The ecology of overwintering among turtles: Where turtles overwinter and its consequences. In Biological Reviews of the Cambridge Philosophical Society (Vol. 81, Issue 3, pp. 339-367). https://doi.org/10.1017/S1464793106007032

Ultsch, G. R., \& Jackson, D. C. (1982). Long-term submergence at 3 degrees C of the turtle Chrysemys picta bellii in normoxic and severely hypoxic water. III. Effects of changes in ambient $\mathrm{PO} 2$ and subsequent air breathing. Journal of Experimental Biology, 97, 87-99. https://doi.org/10.1242/jeb.97.1.87

Vornanen, M., Stecyk, J. A. W., \& Nilsson, G. E. (2009). Chapter 9 The Anoxia-Tolerant Crucian Carp (Carassius Carassius L.). In Fish Physiology (Vol. 27, Issue C, pp. 397-441). https://doi.org/10.1016/S1546-5098(08)00009-5

Walayat, A., Yang, M., \& Xiao, D. (2019). Therapeutic Implication of miRNA in Human Disease. In Antisense Therapy. https://doi.org/10.5772/intechopen. 82738

Wang, D., Zhang, Z., O’Loughlin, E., Lee, T., Houel, S., O’Carroll, D., Tarakhovsky, A., Ahn, N. G., \& Yi, R. (2012). Quantitative functions of argonaute proteins in 
mammalian development. Genes \& Development, 26(7), 693-704.

https://doi.org/10.1101/gad.182758.111

Ward, J. H. (1963). Hierarchical Grouping to Optimize an Objective Function. Journal of the American Statistical Association, 58(301), 236-244.

https://doi.org/10.1080/01621459.1963.10500845

Warnes, G. R., Bolker, B., Bonebakker, L., Gentleman, R., Liaw, W., Lumley, T., \& Al, E. (2015). gplots: Various R programming tools for plotting data. R Package Version 2.17.0. https:/github.com/talgalili/gplots

Warren, D. E., \& Jackson, D. C. (2007). Effects of temperature on anoxic submergence: Skeletal buffering, lactate distribution, and glycogen utilization in the turtle, Trachemys scripta. American Journal of Physiology - Regulatory Integrative and Comparative Physiology, 293(1). https://doi.org/10.1152/ajpregu.00174.2006

Warren, D. E., \& Jackson, D. C. (2008). Lactate metabolism in anoxic turtles: An integrative review. In Journal of Comparative Physiology B: Biochemical, Systemic, and Environmental Physiology (Vol. 178, Issue 2, pp. 133-148). https://doi.org/10.1007/s00360-007-0212-1

Wijenayake, S., Hawkins, L. J., \& Storey, K. B. (2018). Dynamic regulation of six histone $\mathrm{H} 3$ lysine $(\mathrm{K})$ methyltransferases in response to prolonged anoxia exposure in a freshwater turtle. Gene, 649, 50-57. https://doi.org/10.1016/j.gene.2018.01.086

Wijenayake, S., \& Storey, K. B. (2016). The role of DNA methylation during anoxia tolerance in a freshwater turtle (Trachemys scripta elegans). Journal of Comparative Physiology B, 186(3), 333-342.

Wijenayake, S., \& Storey, K. B. (2020). Dynamic regulation of histone H3 lysine (K) acetylation and deacetylation during prolonged oxygen deprivation in a champion anaerobe. Molecular and Cellular Biochemistry, 474(1-2), 229-241. https://doi.org/10.1007/s11010-020-03848-x

Wilczynska, A., Aigueperse, C., Kress, M., Dautry, F., \& Weil, D. (2005). The translational regulator CPEB1 provides a link between dep1 bodies and stress 
granules. Journal of Cell Science, 118(5), 981-992.

https://doi.org/10.1242/jcs.01692

Willmore, W. G., \& Storey, K. B. (1997a). Antioxidant systems and anoxia tolerance in a freshwater turtle Trachemys scripta elegans. Molecular and Cellular Biochemistry, 170(1-2), 177-185. https://doi.org/10.1023/A:1006817806010

Willmore, W. G., \& Storey, K. B. (1997b). Glutathione systems and anoxia tolerance in turtles. American Journal of Physiology - Regulatory Integrative and Comparative Physiology, 273(1 42-1). https://doi.org/10.1152/ajpregu.1997.273.1.r219

Willmore, W. G., \& Storey, K. B. (2007). Purification and properties of glutathione reductase from liver of the anoxia-tolerant turtle, Trachemys scripta elegans. Molecular and Cellular Biochemistry, 297(1-2), 139-149. https://doi.org/10.1007/s11010-006-9339-8

Winter, J., Jung, S., Keller, S., Gregory, R. I., \& Diederichs, S. (2009). Many roads to maturity: microRNA biogenesis pathways and their regulation. Nature Cell Biology, 11(3), 228-234.

Wu, H., Xu, H., Miraglia, L. J., \& Crooke, S. T. (2000). Human RNase III is a 160-kDa protein involved in preribosomal RNA processing. Journal of Biological Chemistry, 275(47), 36957-36965. https://doi.org/10.1074/jbc.M005494200

Yang, S., Maurin, T., Robine, N., Rasmussen, K. D., Jeffrey, K. L., Chandwani, R., Papapetrou, E. P., Sadelain, M., O’Carroll, D., \& Lai, E. C. (2010). Conserved vertebrate mir-451 provides a platform for Dicer-independent, Ago2-mediated microRNA biogenesis. Proceedings of the National Academy of Sciences of the United States of America, 107(34), 15163-15168. https://doi.org/10.1073/pnas.1006432107

Yao, B., Li, S., \& Chan, E. K. L. (2013). Function of GW182 and GW bodies in siRNA and miRNA pathways. Advances in Experimental Medicine and Biology, 768, 7196. https://doi.org/10.1007/978-1-4614-5107-5_6

Yeom, K. H., Lee, Y., Han, J., Suh, M. R., \& Kim, V. N. (2006). Characterization of 
DGCR8/Pasha, the essential cofactor for Drosha in primary miRNA processing. Nucleic Acids Research, 34(16), 4622-4629. https://doi.org/10.1093/nar/gk1458

Zhang, J., Biggar, K. K., \& Storey, K. B. (2013). Regulation of p53 by reversible posttranscriptional and post-translational mechanisms in liver and skeletal muscle of an anoxia tolerant turtle, Trachemys scripta elegans. Gene, 513(1), 147-155. https://doi.org/10.1016/j.gene.2012.10.049

Zhang, J., Hadj-Moussa, H., \& Storey, K. B. (2016). Current progress of high-throughput microRNA differential expression analysis and random forest gene selection for model and non-model systems: an R implementation. Journal of Integrative Bioinformatics, 13(5), 306. https://doi.org/10.2390/biecoll-jib-2016-306

Zhang, J., \& Storey, K. B. (2016). RBioplot: An easy-to-use R pipeline for automated statistical analysis and data visualization in molecular biology and biochemistry. PeerJ, 2016(9). https://doi.org/10.7717/peerj.2436

Zhang, J., \& Storey, K. B. (2018). RBiomirGS: An all-in-one miRNA gene set analysis solution featuring target mRNA mapping and expression profile integration. PeerJ, 2018(1). https://doi.org/10.7717/peerj.4262

Ziello, J. E., Jovin, I. S., \& Huang, Y. (2007). Hypoxia-Inducible Factor (HIF)-1 regulatory pathway and its potential for therapeutic intervention in malignancy and ischemia. In Yale Journal of Biology and Medicine (Vol. 80, Issue 2, pp. 51-60).

Zlotorynski, E. (2019). Insights into the kinetics of microRNA biogenesis and turnover. Nature Reviews Molecular Cell Biology, 20(9), 511. https://doi.org/10.1038/s41580019-0164-9 
APPENDICES 
APPENDIX A

\section{Publications}




\section{PUBLICATIONS [6]}

Articles published in peer-reviewed journals [2]

1. Tessier, S.N., Breedon, S.A., and Storey, K.B. (2021) Modulating Nrf2 transcription factor activity: revealing the regulatory mechanisms of antioxidant defenses during hibernation in thirteen-lined ground squirrels. Cell Biochemistry \& Function.

2. Breedon, S.A., Hadj-Moussa, H.M., and Storey, K.B. (2021) Nrf2 activates antioxidant enzymes in the anoxic red-eared slider turtle, Trachemys scripta elegans. Journal of Experimental Zoology Part A: Ecology and Integrative Physiology.

Articles awaiting submission to peer-reviewed journals [4]

3. Breedon, S.A., Gupta, A., and Storey, K.B. Regulation of apoptosis and autophagy during anoxia in the freshwater crayfish, Orconectes virilism.

4. Gupta, A., Breedon, S.A., and Storey, K.B. Activation of the tumor suppressor protein, p53 in the anoxia resistant crayfish, Orconectes virilism.

5. Breedon, S.A. and Storey, K.B. Expression of Bcl-2 family proteins and antiapoptotic defense in red-eared slider turtles, Trachemys scripta elegans.

6. Allan, M.A., Breedon, S.A., and Storey, K.B. Expression of NF-кB and downstream antioxidant genes in liver of hibernating ground squirrels. 


\section{APPENDIX B}

\section{Antibody Supplier Information}


Table B1. Antibody information and suppliers.

\begin{tabular}{|c|c|c|c|}
\hline Antibody & Company & Catalogue Number & Secondary \\
\hline AGO1 & GeneTex & GTX47799 & Anti-Rabbit \\
\hline AGO2 & ECM Biosciences & AP5281 & Anti-Rabbit \\
\hline p-AGO2 $2^{\text {Tyr393 }}$ & ECM Biosciences & AP5311 & Anti-Rabbit \\
\hline p-AGO2 $2^{\operatorname{Ser} 387}$ & ECM Biosciences & AP5291 & Anti-Rabbit \\
\hline AGO3 & $\mathrm{AbCam}$ & AB3593 & Anti-Rabbit \\
\hline AGO4 & AbCam & AB85077 & Anti-Rabbit \\
\hline DGCR8 & GeneTex & GTX130061 & Anti-Rabbit \\
\hline DICER & AbClonal & A6021 & Anti-Rabbit \\
\hline DROSHA & GeneTex & GTX56186 & Anti-Rabbit \\
\hline GW182 & AbClonal & A6115 & Anti-Rabbit \\
\hline PABPC1 & AbClonal & A14872 & Anti-Rabbit \\
\hline PACT & GeneTex & GTX114215 & Anti-Rabbit \\
\hline RAN-GTP & GeneTex & GTX114139 & Anti-Rabbit \\
\hline TIAR & Santa Cruz & SC398372 & Anti-Mouse \\
\hline TIA-1 & Santa Cruz & $\mathrm{SC} 1751$ & Anti-Goat \\
\hline TRBP & GeneTex & GTX48546 & Anti-Rabbit \\
\hline XPO5 & GeneTex & GTX130727 & Anti-Rabbit \\
\hline XRN1 & Santa Cruz & SC165985 & Anti- Mouse \\
\hline
\end{tabular}


APPENDIX C

\section{Verifying Antibody Specificity for Immunoblotting}


The following steps were taken to ensure that all of the antibodies used in this thesis crossreacted with the proteins of interest at the appropriate molecular weight:

1. When manufacturers supplied the epitope sequence of their antibodies, this was aligned against the red-eared slider turtle genome using National Center for $\begin{array}{lllll}\text { Biotechnology's } & \text { (NCBI) } & \text { BLAST } & \text { function } & \text { (NCBI; }\end{array}$ https://blast.ncbi.nlm.nih.gov/Blast.cgi). When antibodies from manufacturers who did not supply the antibody's epitope sequence were used, the target protein sequences from various species (human, mouse, chicken, red-eared slider turtle, Chinese soft-shelled turtle, and Xenopus laevis) were aligned using the Clustal Omega multiple sequence alignment program (EMBL-EBI, https://www.ebi.ac.uk/Tools/msa/clustalo/) to determine sequence conservation. If the sequences were highly conserved across species, it was assumed that the antibody would likely cross-react in red-eared slider turtle.

2. Where possible, polyclonal antibodies that were tested on numerous species were used to increase the likelihood of cross-reactivity in the red-eared slider turtle.

3. Immunoblot membranes were incubated with nonfat milk prior to primary antibody probing in order to minimize non-specific background binding.

4. All antibodies were tested with a mammalian positive control sample (thirteenlined ground squirrel) to verify that the antibodies cross-reacted at the expected molecular weight. 
APPENDIX D

Optimized Immunoblotting Conditions 
Table D1. Optimized immunoblotting conditions for liver and muscle western blots.

\begin{tabular}{|c|c|c|c|c|c|c|}
\hline Target & $\begin{array}{c}\text { Gel } \\
\%\end{array}$ & $\begin{array}{c}\text { Protein } \\
\text { loaded } \\
(\mu g)\end{array}$ & $\begin{array}{l}\text { Run time } \\
\text { (min) }\end{array}$ & $\begin{array}{c}\text { Transfer } \\
\text { amperage } \\
(\mathrm{mA})\end{array}$ & $\begin{array}{c}\text { Transfer } \\
\text { time } \\
\text { (min) }\end{array}$ & $\begin{array}{c}\text { Milk } \\
\text { block \% }\end{array}$ \\
\hline AGO1 & 8 & 40 & 100 & 180 & 150 & 8 \\
\hline AGO2 & 8 & 40 & 80 & 180 & 150 & 8 \\
\hline p-AGO2 $2^{\text {Tyr393 }}$ & 8 & 25 & 80 & 180 & 150 & 8 \\
\hline p-AGO2 $2^{\operatorname{Ser} 387}$ & 8 & 25 & 80 & 180 & 150 & 5 \\
\hline AGO3 & 8 & 40 & 80 & 180 & 150 & 8 \\
\hline AGO4 & 8 & 40 & 80 & 180 & 150 & 8 \\
\hline DGCR8 & 8 & 25 & 80 & 180 & 150 & 5 \\
\hline DICER & 6 & 25 & 80 & 50 & 900 & 2 \\
\hline DROSHA & 8 & 25 & 100 & 180 & 150 & 8 \\
\hline GW182 & 6 & 25 & 80 & 50 & 900 & 3 \\
\hline PABPC1 & 12 & 25 & 70 & 180 & 90 & 3 \\
\hline PACT & 12 & 25 & 50 & 180 & 150 & 5 \\
\hline RAN-GTP & 15 & 25 & 72 & 180 & 150 & 8 \\
\hline TIAR & 12 & 25 & 70 & 180 & 90 & 3 \\
\hline TIA-1 & 12 & 25 & 70 & 180 & 90 & 3 \\
\hline TRBP & 12 & 25 & 50 & 180 & 150 & 5 \\
\hline XPO5 & 8 & 25 & 100 & 180 & 150 & 8 \\
\hline XRN1 & 6 & 25 & 80 & 50 & 900 & 3 \\
\hline
\end{tabular}




\section{APPENDIX E}

Representative Immunoblot Images 
A

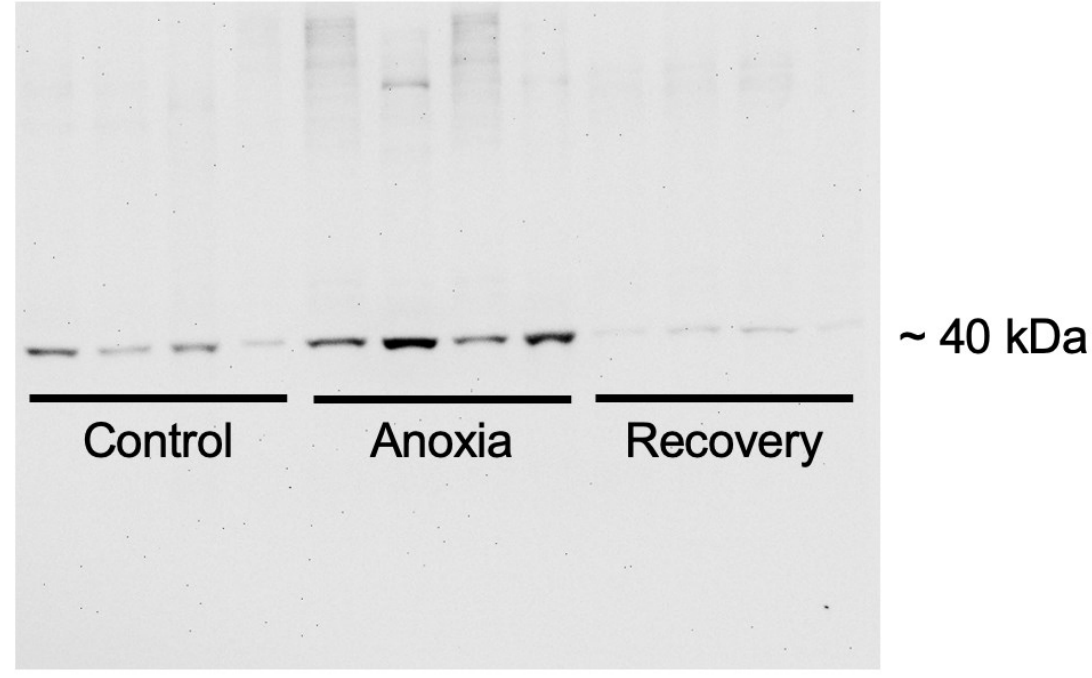

B

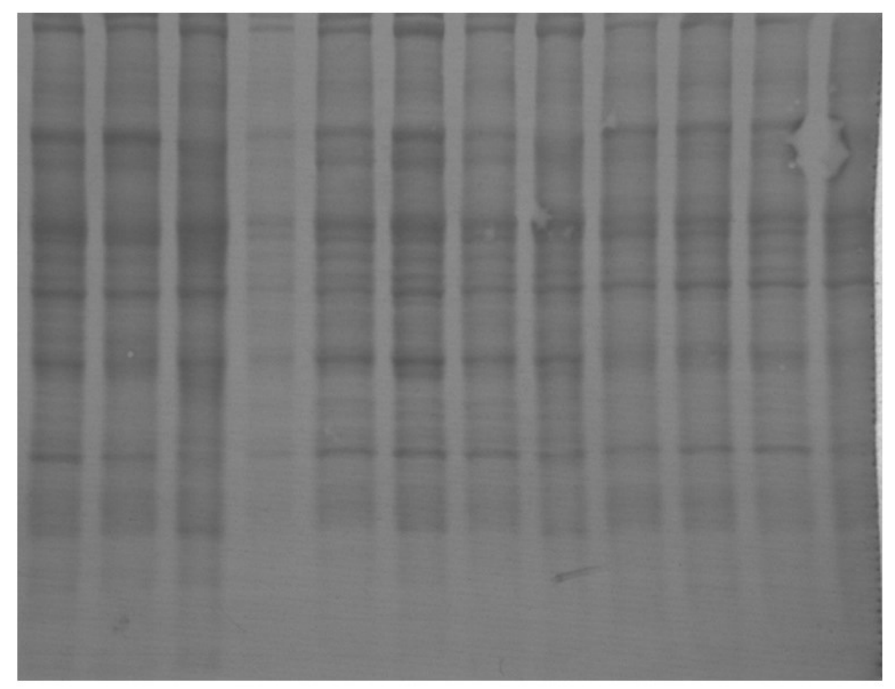

Figure E1. (A) Representative whole blot of TRBP expression and (B) corresponding Coomassie stained membrane in red-eared slider turtle liver under normoxic control, anoxia, and recovery conditions. 


\section{A}
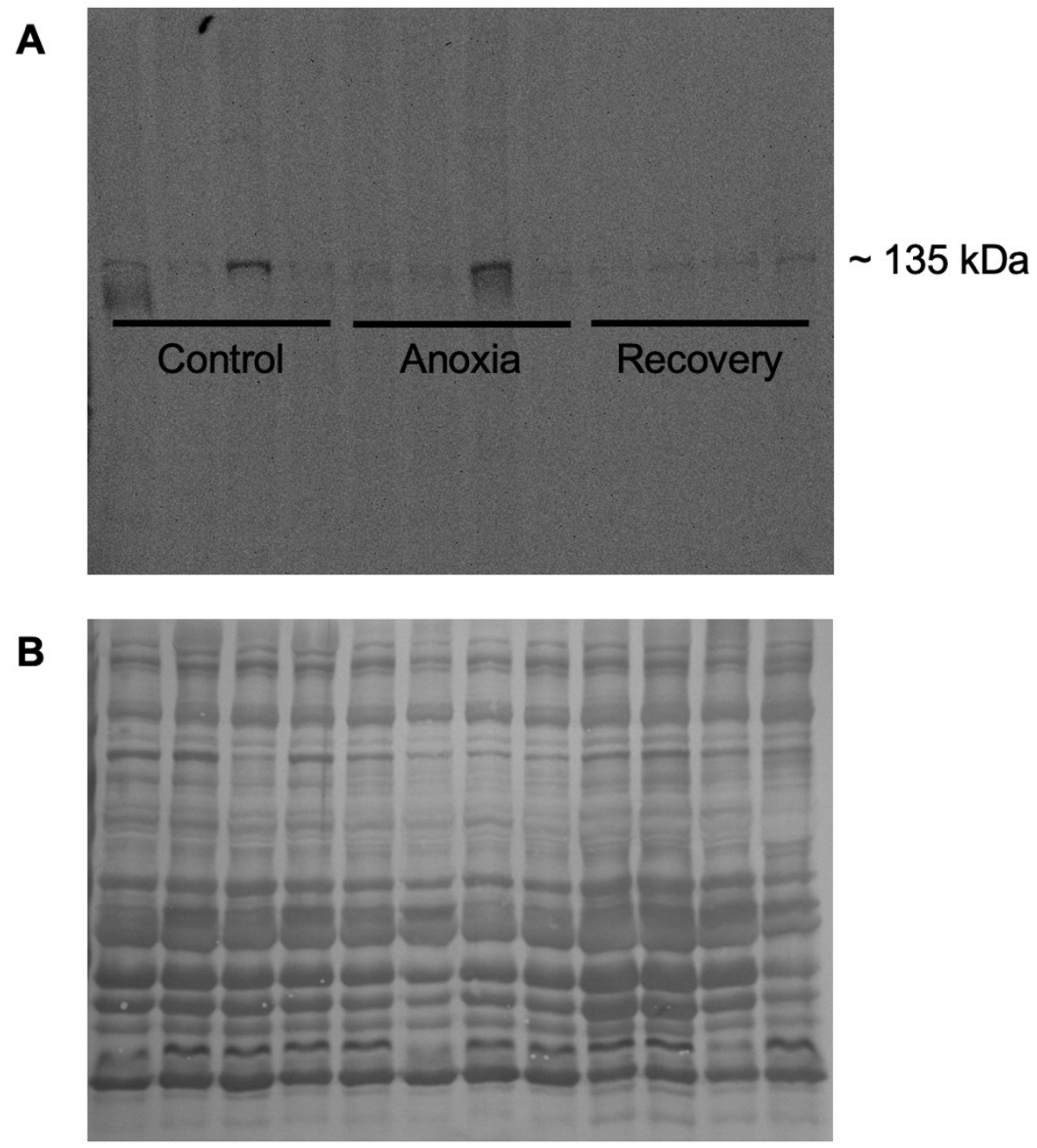

Figure E1. (A) Representative whole blot of XPO5 expression and (B) corresponding Coomassie stained membrane in red-eared slider turtle red muscle under normoxic control, anoxia, and recovery conditions. 

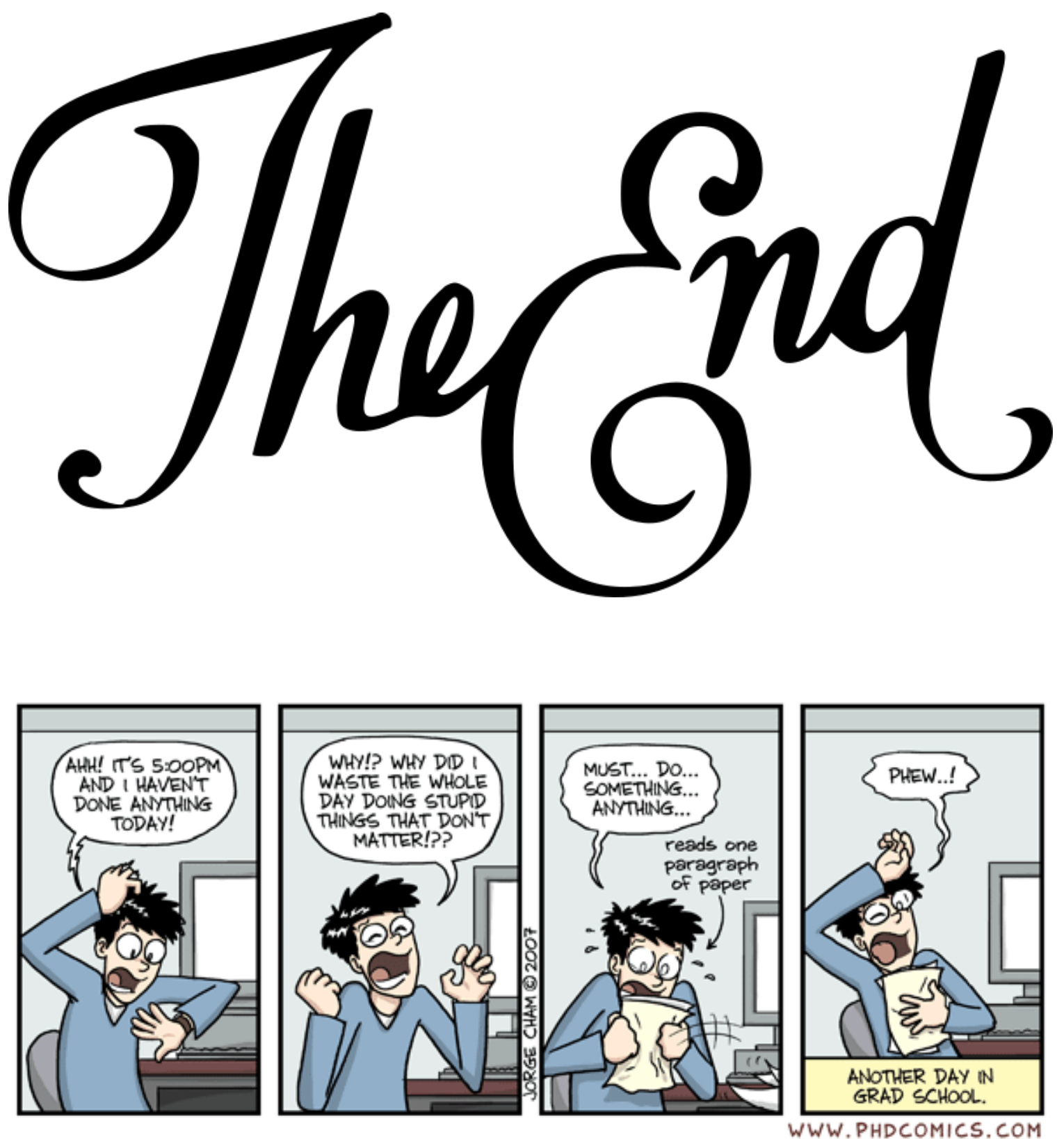

"Done is better than perfect."

- Sheryl Sandberg 